\title{
Geração de malhas volumétricas a partir de imagens
}

\author{
Alex Jesús Cuadros Vargas
}





\title{
Geração de malhas volumétricas a partir de imagens
}

\author{
Alex Jesús Cuadros Vargas
}

Orientador: Prof. Dr. Luis Gustavo Nonato

Tese apresentada ao Instituto de Ciências Matemáticas e de Computação - ICMC-USP, como parte dos requisitos para obtenção do título de Doutor em Ciências - Ciências de Computação e Matemática Computacional. 
Aos meus pais Ernesto e Yolanda 


\section{Agradecimentos}

Gostaria de agradecer:

- Aos meus pais Ernesto e Yolanda e aos meus irmãos Tito e Chepy, por todo o apoio e carinho que sempre recebi deles.

- Ao meu orientador Luis Gustavo Nonato, por todo seu apoio, desde o primeiro momento do doutorado, pela confiança depositada em mim, e por todos os seus conselhos.

- Gostaria também de agradecer aos professores João Batista, Antonio Castelo, Rosane Mimghim, Maria Cristina Ferreira de Oliveira e José Alberto Cuminato, pelos seus conselhos e disposição de ajudar durante este período.

- Aos meus colegas, Prof. Valdemir, João, Cassio, Helton, Tiago, Igor, Eduardo, Edwin, Alexandre, Kemelli, Mario, Marcello, Leandro, Claudio e Alex, pela sua amizade e aportes a este trabalho.

- À Fundação de Amparo à Pesquisa do Estado de São Paulo (FAPESP), pelo apoio econômico da bolsa de doutorado (Processo Nro. 02/05243-4). 



\section{Resumo}

Técnicas para gerar malhas triangulares ou tetraedrais a partir de imagens, ou assumem como entrada uma imagem pré-processada, ou geram uma malha sem distinguir as diferentes estruturas contidas na imagem. O pré-processamento e a ausência de estruturas bem definidas podem apresentar dificuldades na utilização das malhas geradas em algumas aplicações, tais como simulações numéricas. Neste trabalho, apresentamos uma nova técnica que elimina a necessidade do pré-processamento embutindo a segmentação dentro do processo de geração de malha. Além disto, a técnica proposta considera critérios de qualidade nas malhas geradas, mostrando-se apropriada para aplicações de simulação numérica assim como modelagem de imagens com malhas. 



\section{Abstract}

Techniques devoted to generate triangular or tetrahedral meshes from images either take as starting point a pre-processed image or generate a mesh without distinguishing different structures contained in the image. The pre-process and the absence of well defined structures may impose difficulties in using the resulting mesh in some applications as, for example, numerical simulations. In this work, we present a new technique that aims at eliminating the need for pre-processing by building the segmentation into the mesh generation process. Furthermore, the proposed technique consider quality criteria in the generated meshes, being appropriated for applications in numerical simulation as well as in image modeling. 



\section{Sumário}

Lista de Figuras . . . . . . . . . . . . . . . . . . . . . . . . . xii

Lista de Tabelas . . . . . . . . . . . . . . . . . . . . xiii

1 Introdução $\quad 1$

2 Trabalhos relacionados e Contextualização do problema 5

2.1 Abordagens da literatura . . . . . . . . . . . . . . . . . . 5 5

2.2 Contextualização da proposta . . . . . . . . . . . . . . . . . . 9

3 Conceitos relacionados $\quad 11$

3.1 Complexos Simpliciais, Triangulação de Delaunay e Diagrama de Voronoi . . 11

3.2 Refinamento de malhas Delaunay . . . . . . . . . . . . . . . . . . . 14

3.2.1 O problema clássico de gerar malhas . . . . . . . . . . . . . . . 14

3.2.2 Qualidade de triângulos . . . . . . . . . . . . . . 15

3.2 .3 Algoritmo de Chew . . . . . . . . . . . . . . . . . . . . . 17

3.2 .4 Algoritmo de Ruppert . . . . . . . . . . . . . . . . . . . 18

3.2.5 Considerações sobre algoritmos de refinamento Delaunay bidimensionais 20

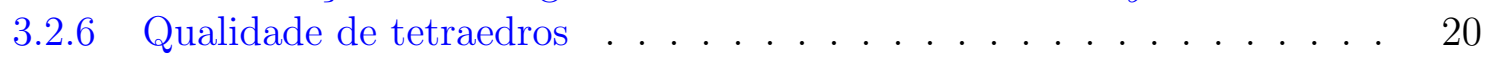

3.2.7 Algoritmo de Shewchuk . . . . . . . . . . . . . . . . 22

3.2.8 Considerações sobre algoritmos de refinamento Delaunay tridimensionais 23

3.3 Estratégia de segmentação EM/MPM . . . . . . . . . . . . . . . . . . . 24

4 O algoritmo Imesh $\quad 29$

4.1 Construção de malha . . . . . . . . . . . . . . . . . . . . . . . . . . . . . . . . . . 31

4.1 .1 Cálculo do erro pontual . . . . . . . . . . . . . . . . 33

4.1.2 Cálculo do ponto mais distante dos vértices da triangulação . . . . . 36

4.1.3 Detalhes e complexidade do processo de construção de malha . . . . . 37

4.2 Particionamento de malha . . . . . . . . . . . . . . . . . . . . . 39

4.2.1 Características da malha a ser particionada . . . . . . . . . . 39

4.2.2 Particionamento por operadores de agrupamento (POpA) . . . . . . 41

4.2.3 Particionamento por campos aleatórios Markovianos (PCAM) . . . . 53

4.2.4 Detalhes e complexidade do processo de particionamento . . . . . . . 57

4.3 Melhoramento de qualidade de malha . . . . . . . . . . . . . . . . 58 
4.3.1 Refinamento . . . . . . . . . . . . . . . . . 59

4.3.2 Manutenção da rotulação . . . . . . . . . . . . . . . . . . 61

4.3.3 Detalhes e complexidade do processo de refinamento de qualidade de malha .......................... 63

5 Resultados $\quad 65$

5.1 Resultados bidimensionais . . . . . . . . . . . . . . . . . . 65

5.2 Resultados tridimensionais . . . . . . . . . . . . . . . . . . . . 69

6 Conclusões e trabalhos futuros $\quad 89$ 


\section{Lista de Figuras}

3.1 Triangulação de Delaunay e Diagrama de Voronoi (Exemplo bidimensional). 13

3.2 Triângulo e medidas de qualidade . . . . . . . . . . . . . . . . . . 16

3.3 Imagem que demonstra $d=2 * r * \sin (\theta) \ldots \ldots \ldots \ldots$

3.4 Resultados de exemplo dos algoritmos de refinamento Delaunay de Chew e Ruppert (Malhas geradas pelo Bern Eppstein Gilbert) . . . . . . . . . . . . .

3.5 O problema de ângulos pequenos e seu tratamento. (Imagens extraídas do trabalho de Shewchuk (2005)) . . . . . . . . . . . . . . . . . . . . . . . . .

3.6 Tetraedros considerados ruins. . . . . . . . . . . . . . . . . . . 22

3.7 Resultados de exemplo do algoritmo de refinamento de Shewchuk (Imagens extraídas do trabalho de Shewchuk (2005). . . . . . . . . . . . . .

4.1 Medida de erro pontual de homogeneidade. No exemplo bidimensional (fila de cima), os círculos representam o conjunto $P^{h_{j}}$ de pontos selecionados sobre as medianas de uma célula . O quadrado representa o ponto escolhido deste conjunto com o qual o erro da célula $a_{d}$ é calculado. Logo, a idéia é estendida diretamente para o exemplo 3D (fila de abaixo) . . . . . . . . . . . . . . . 35

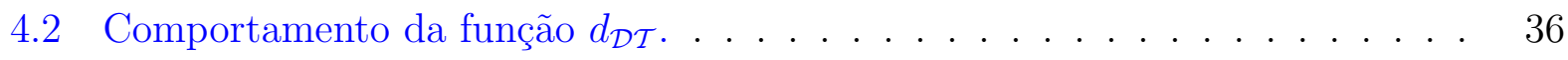

4.3 Comparações entre os critérios de cálculos de distância, Figuras (b) e c(); e comparações entre cálculos de erro pontual, Figuras (d) (e) e (f). . . . . . . 38

4.4 Células com ruído. . . . . . . . . . . . . . . . . . . . . 42

4.5 Comportamento da função $\mathscr{S}_{I}$ ao comparar pares de sub-malhas com padrões de cor com muita e pouca similaridade. . . . . . . . . . . . . . .

4.6 Comportamento da função $\gamma$ para estabelecer a relevância de duas sub-malhas, uma grande com várias células ${ }_{d}$ (distância maior) e uma de área pequena com poucas células $_{d}$ (distância menor). . . . . . . . . . . . . . . . . . 45

4.7 Particionamento utilizando o operador ASmV . . . . . . . . . . . . . . . . . . . . . . . . . . . . . 46

4.8 Particionamento utilizando o operador ASmS. . . . . . . . . . . . . . . . . . 47

4.9 Conduta da função $\mathscr{S}_{G}$ ao comparar dois triângulos. . . . . . . . . . . . . . . . . . . . . . . . . . . . . . . .

4.10 Particionamento utilizando o operador ACG. . . . . . . . . . . . . . . . 50

4.11 Particionamento utilizando barreira de restrição. . . . . . . . . . . . . . . 54 
4.12 Particionamento utilizando o operador a abordagem PCAM. Fatia de um crânio de macaco obtida por tomografia computadorizada . . . . . . . . . . .

4.13 Etapa de refinamentod qualiade. Malhas obtidas com uma restrição de ângulo mínimo de $30^{\circ} \ldots \ldots \ldots \ldots \ldots \ldots$

5.1 Comparação dos particionamentos obtidos pelas abordagens PCAM e POpA, em malhas obtidas por funções de erro pontual de interpolação e de homogeneidade. . . . . . . . . . . . . . . . . . . 70

5.2 Comparação dos erros de interpolação. . . . . . . . . . . . . . . . . . . . 73

5.3 Imagens geradas com interpolação de ordem zero. . . . . . . . . . . . . . . . 74

5.4 Interação entre alguns dos operadores da estratégia POpA e importância da geometria como critério de particionamento. . . . . . . . . . . 75

5.5 Processo completo do algoritmo Imesh bidimensional. . . . . . . . . . . . . . 76

5.6 Gerações de malha de qualidade. . . . . . . . . . . . . . . . . 77

5.7 Teste de deformação utilizando malhas elásticas, a partir de uma imagem bidimensional. . . . . . . . . . . . . . . . . . 78

5.8 Outros resultados bidimensionais obtidos. . . . . . . . . . . . . . . . 79

5.9 Particionamento tridimensional com critérios geométricos. . . . . . . . . 80

5.10 Exemplo de utilização de operadores com barreira de restrição. . . . . . . . . 81

5.11 Exemplo de tratamento uso pela abordagem PCAM. . . . . . . . . . . . 82

5.12 Exemplos de resultados com um número alto de sub-malhas, obtido . . . . . 83

5.13 Exemplo 1 de refinamento tridimensional. Foram utilizados $\mathscr{E}_{v}, B=2$ e $\Xi=5.84$

5.14 Exemplo 2 de refinamento tridimensional. Foram utilizados $\mathscr{E}_{v}, B=2$ e $\Xi=8.85$

5.15 Exemplo 3 de refinamento tridimensional. Foram utilizados $\mathscr{E}_{v}, B=2$ e $\Xi=6 . \quad 86$

5.16 Outros particionamentos tridimensionais obtidos . . . . . . . . . . 87

5.17 Outras visualizações de malhas via rendering volumétrico realizadas. . . . . 88 


\section{Lista de Tabelas}

5.1 Porcentagem de pixels classificados errados no particionamento. . . . . . . . 67

5.2 Erro médio das interpolações. . . . . . . . . . . . . . . . . . . . . . 67

5.3 Tempos computacionais (em segundos) . . . . . . . . . . . . . . 69

5.4 Tempos computacionais para o algoritmo tridimensional(em segundos) . . . 72 



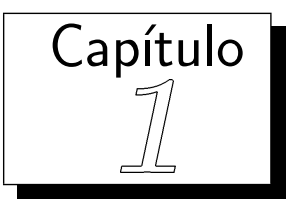

\section{Introdução}

O problema de gerar malhas diretamente a partir de imagens tem atraído a atenção de muitos pesquisadores nos últimos anos. O interesse por tal assunto é motivado pelo avanço de dispositivos não invasivos, tais como Tomografia Computadorizada (CT-Computed Tomography), Ressonância Magnética (MRI-Magnetic Resonance Imaging), entre outros, os quais são capazes de produzir imagens contendo informações de um objeto tridimensional. Outro fator que tem incentivado o desenvolvimento de técnicas eficientes para obtenção de modelos tridimensionais a partir de dados volumétricos é o desenvolvimento de aplicações computacionais que fazem uso destes modelos como, por exemplo, simulação cirúrgica (Bielser e Gross., 2000; Shahidi et al., 1998), simulação numérica de fenômenos físicos (Pescatore et al., 2001) e análise não invasiva de estruturas internas (Minghim et al., 2002; Ferrant et al., 2001).

Tradicionalmente, várias etapas estão envolvidas na tarefa de construir a partir de imagens, malhas apropriadas para fins de simulações numéricas. Por exemplo, uma seqüência de passos comumente utilizada é: pré-processamento (Sonka, 1999)(eliminação de ruído, suavização, segmentação, binarização, etc.), extração de contornos (Haralick e Shapiro, 1992), geração de superfícies a partir de contornos (Geiger, 1993a; Cheng e Dey, 1999; Nonato et al., 2005) e geração de malhas tetraedrais com critérios de qualidade a partir de superfícies (Shewchuk, 1997; Alliez et al., 2005).

Nos últimos anos, um grande esforço tem sido feito no sentido de criar algoritmos 
capazes de atuar diretamente em imagens, evitando-se assim algumas etapas tradicionais. Podemos agrupar tais técnicas em duas classes principais. A primeira classe de algoritmos visa a geração da malha diretamente das imagens de entrada, evitando-se o exaustivo préprocessamento. Porém, estas técnicas, em geral, representam a imagem de entrada com a malha produzida, sem separar objetos presentes na imagem, o que muitas vezes inviabiliza seu uso em simulações numéricas. A segunda classe de técnicas tem como objetivo produzir malhas apropriadas para fins de simulação. No entanto, estas técnicas não dispensam uma etapa prévia de segmentação da imagen de entrada a fim de definir precisamente objetos contidos na imagem.

Os dois grupos de técnicas possuem vantagens e desvantagens dependendo da aplicação. Por exemplo, se o objetivo é apenas visualizar o conteúdo da imagem a partir da malha gerada, uma boa opção seria empregar uma técnica de representação de imagens, como as técnicas do primeiro grupo. Por outro lado, se o objetivo é realizar simulações numéricas empregando modelos tridimensionais precisos, como em escoamento de fluidos por exemplo, então o mais apropriado seria utilizar idéias do segundo grupo de técnicas.

Neste trabalho apresentamos uma nova abordagem, chamada Imesh, que busca combinar características de ambos grupos de abordagens descritos acima. Diferentemente das abordagens descritas na literatura, a técnica Imesh assume como entrada uma imagem não pré-processada e procura gerar uma malha segmentada, considerando inclusive critérios de qualidade nos elementos da malha. A idéia fundamental da abordagem Imesh é dividir o problema, de geração de malhas de qualidade a partir de imagens em três etapas principais, as quais são denominadas: Construção de malha, Particionamento de malha e Refinamento de qualidade de malha. Na primeira etapa, Construção de malha, representa-se a imagem de entrada (não pré-processada) com uma malha Delaunay. Este processo de aproximação da imagem pela malha pode considerar distintos critérios de erro, flexibilizando a criação de malhas para diferentes aplicações. Ao final da primeira etapa a imagem de entrada fica representada por meio de padrões de cor calculados em cada elemento da malha. O objetivo da segunda etapa, Particionamento de malha, é separar a malha obtida em um número desejado de sub-malhas. Esta etapa utiliza, além dos padrões de cor obtidos da imagem, propriedades topológicas e geométricas da malha como informação adicional para realizar o processo de particionamento. Finalmente, a etapa de Refinamento de qualidade de malha, refina as submalhas geradas respeitando suas fronteiras e garantindo alguns critérios de qualidade nos 
elementos simplicias. Muitas aplicações importantes como a simulação de válvulas cardíacas (Keener e Panfilov., 1995), escoamento sanguíneo (Dari et al., 2000) e simulação cirúrgica (Auer et al., 1999), necessitam de um método de geração de malha como o apresentado neste trabalho.

Vale ressaltar que poucos trabalhos descritos na literatura abordam o problema de geração de malha a partir de imagens de forma semelhante ao Imesh, uma vez que praticamente todos os geradores de malha a partir de imagens realizam um pré-processamento nas imagens de entrada antes do processo de geração de malha propriamente dito (Zhang et al., 2003; Hale, 2001a; Cebral e Lohner, 1999a). Outro objetivo do trabalho aqui proposto é considerar critérios teóricos de qualidade aos elementos da malha gerada (Shewchuk, 2002b; A. Liu, 1994; Knupp, 2001; Lo., 1997; Bern e Eppstein, 1992; Chew, 1989; B. S.Baker, 1988). Salientamos que o ferramental básico empregado na técnica Imesh é a triangulação de Delaunay. Dentre as razões para esta escolha está a existência de algoritmos robustos para a construção de tais estruturas (Fabri et al., 2000; Boissonnat et al., 2002; Fortune, 1994; Edelsbrunner, 2000). Outro aspecto importante que reforça o uso da triangulação de Delaunay é o desenvolvimento teórico referente aos algoritmos de refinamento (Chew, 1993; Ruppert, 1995; Shewchuk, 1999) e melhoria da qualidade das malhas Delaunay (Li, 2000; Edelsbrunner et al., 2000; Cheng e Dey, 2002; Cheng et al., 2000).

\section{Organização do texto}

Organizamos este trabalho como descrito a seguir. O Capítulo 2 apresenta uma discussão sobre métodos relacionados à geração de malhas a partir de imagens. Seguidamente, o Capítulo 3 descreve conceitos importantes e abordagens que serão de utilidade para os Capítulos seguintes. A técnica Imesh, proposta neste trabalho, é detalhada no Capítulo 4. O Capítulo 5 apresenta os resultados obtidos. Finalmente, o Capítulo 6 mostra as conclusões e discute sobre trabalhos futuros. 


\section{Trabalhos relacionados e Contextualização do problema}

Neste Capítulo discute-se diferentes abordagens que têm sido propostas na literatura para atacar o problema de criação de malhas a partir de imagens. Tal discução será realizada na Seção 2.1. Na Seção 2.2 será dada uma contextualização do algoritmo Imesh proposto neste trabalho.

\subsection{Abordagens da literatura}

Métodos que buscam gerar malhas a partir de imagens podem ser divididos em duas categorias diferentes: abordagens de Modelamento de Imagens por meio de Malhas e abordagens de Geração de Malhas a partir de Imagens para Simulação. O grupo de técnicas de modelagem de imagens por meio de malhas, procuram construir malhas somente para representar a imagem original, por exemplo, buscando uma compressão da mesma. Por outro lado, o grupo de métodos que busca geração de malhas a partir de imagens para simulação, visam resultados nos quais elementos da malha estejam alinhados às características da imagem de entrada, tentando também detectar precisamente distintas estruturas internas. Técnicas pertencentes a estes dois grupos são descritas a seguir. 
Modelamento de Imagens por meio de Malhas

Este tipo de técnicas de geração de malha é pouco dependente de uma etapa inicial de pré-processamendo e tem surgido no contexto de modelagem de imagem. Embora não visem reconstruir os objetos contidos nas imagens, o ferramental empregado por tais métodos pode ser de grande valia em reconstrução a partir de volumes. Um dos primeiros trabalhos nesse contexto foi proposto por Terzopoulos e Vasilescu (1992), que aborda o problema por meio de malhas adaptativas representando um sistema tipo massa-mola. A idéia do método é mover os vértices da malha com base em informações de gradiente e curvatura de modo a concentrar vértices em regiões do volume com rápida variação de intensidade. Uma abordagem semelhante, denominada Atomic Mesh, foi proposta por Hale (2001b). Nessa abordagem, uma função potencial é empregada com o objetivo de alinhar um conjunto de vértices, originalmente posicionados em um grid regular, com características da imagem. Uma vez posicionados, os vértices servem de base para a construção da malha final por meio de uma triangulação de Delaunay. Embora não utilizem binarização, os métodos acima são sensíveis a ruído, sendo necessário uma etapa de filtragem nos dados originais.

Técnicas baseadas em minimização de erro têm também sido largamente empregadas no contexto de modelagem de imagem. Tais métodos buscam distribuir os vértices da malha de modo a minimizar o erro de interpolação entre os dados armazenados na malha e a imagem original. Um exemplo desse tipo de abordagem é o método iterativo proposto por Garland e Heckbert (1995), o qual insere, na triangulação de Delaunay, os pontos onde o erro de interpolação é máximo. Na verdade, estratégias iterativas de inserção de vértices têm sido adotadas por vários autores, sendo a escolha da função de erro e a estratégia de refinamento, os principais diferenciais dos trabalhos. O trabalho de Grosso et al. (1997), por exemplo, guia o processo de refinamento por meio de uma medida de erro derivada de aproximação por mínimos quadrados, sendo a subdivisão dos elementos da malha baseada em um conjunto de regras de particionamento. A estratégia proposta por Roxborough e Nielson (2000) também utiliza mínimos quadrados para estimar o erro de interpolação, mas, diferente da abordagem anterior, os autores adotam uma única estratégia de subdivisão de elementos baseada na maior aresta do tetraedro. Uma abordagem iterativa mais sofisticada foi proposta por Takahashi et al. (2004), fazendo uso de critérios topológicos e geométricos na concepção das regras de criação e refinamento da malha. 
Alguns métodos iterativos, como o proposto por García et al. (1999), empregam medidas de erro de interpolação juntamente com informações como gradiente e curvatura para derivar a estratégia de refinamento. Isto é, maior quantidade de vértices são dispostos em áreas com curvaturas elevadas. O modelo da malha é construído inserindo os vértices escolhidos em uma triangulação de Delaunay. Vale notar que a estratégia de subdivisão de células afeta diretamente a qualidade da aproximação gerada, sendo esse um ponto importante observado pelos autores. Um bom levantamento sobre estratégias de subdivisão, juntamente com os artefatos produzidos por elas, pode ser encontrado no trabalho de Carr et al. (2006). Vários outros algoritmos que representam imagens usando malhas são também baseados em métodos iterativos (Huang e Hsu, 1994; Gevers e Smeulders, 1997; Coleman e Scotney, 2005). Alternativamente, algumas técnicas têm adotado uma estratégia oposta, por exemplo, uma malha fina é sucessivamente simplificada até que o erro de aproximação alcance uma tolerância (Ciampalini et al., 1997). Abordagens mistas que combinam refinamento e simplificação também têm sido propostas em outros trabalhos (Kocharoen et al., 2005; Pedrini, 2001).

Métodos não iterativos, mas que fazem uso do erro de interpolação, também têm sido descritos na literatura. Por exemplo, Yang et al. (2003) utilizam zero-crossing em conjunto com a estratégia de difusão de erro de Floyd-Steinberg para escolher um conjunto de pontos a partir dos quais uma triangulação de Delaunay é calculada. O erro de interpolação é, de fato, bastante reduzido com essa abordagem, a qual resulta ainda em malhas de boa qualidade.

Uma importante característica dos métodos de modelagem de imagem é a não dependência de pré-processamento. Por outro lado, as malhas geradas podem não se ajustar aos objetos de interesse contidos nas imagens, o que dificulta o uso imediato de tais métodos no contexto de simulação.

\section{Geração de Malhas a partir de Imagens para Simulação}

Este grupo de técnicas é muito dependente de uma etapa de pré-processamendo na imagem de entrada, pois visa distinguir objetos contidos nas imagens de entrada e gerar malhas apropriadas para fins de simulação numérica.

Grande parte destes métodos de construção de malhas utilizam abordagens por funções implícitas, principalmente utilizando iso-valores das imagens, os quais são empregados para definir as superfícies de nível que aproximam a fronteira dos objetos de interesse. 


\section{CAPÍTULO 2. TRABALHOS RELACIONADOS E CONTEXTUALIZAÇÃO DO PROBLEMA}

Estratégias implícitas têm sido muito utilizadas por métodos de reconstrução que visam produzir modelos apropriados para fins de simulação numérica. Por exemplo, o algoritmo proposto por Cebral e Lohner (1999b), assume um pesado esquema de pré-processamendo que inclui filtros estatísticos, preenchimento de buracos, intervenção do usuário e finalmente binarização, facilitando assim a definição da iso-superfície. Os autores propõem ainda o emprego de um esquema de avanço de fronteira para gerar malhas volumétricas simpliciais adequadas para simulação numérica.

As abordagens apresentadas por Zhang et al. (2003) e Berti (2004) são baseadas em octrees, considerando também etapas prévias de pré-processamendo. Zhang et al. aplicam primeiro um passo de eliminação de ruído no volume de entrada. Depois, duas iso-superfícies são selecionadas e o espaço existente entre elas é considerado como a região onde será criada a malha. A partir deste intervalo, uma octree é construída para guiar a criação de uma malha adaptativa de tetraedros ou octaedros. Finalmente a qualidade da malha é melhorada com operações de contração de aresta. A técnica de Berti começa realizando um processo de segmentação na imagem de entrada, restringido-se a gerar somente duas regiões. Uma octree é criada a partir da fronteira definida pela segmentação, e uma malha tetraedral é criada utilizando-se a octree e uma variação volumétrica do algoritmo marching cubes. Por fim, o modelo é melhorado aplicando-se operações de eliminação, movimento e suavização nos vértices da malha.

Além das abordagens descritas acima, existem ainda métodos voltados para a reconstrução visual dos objetos, como as técnicas de visualização volumétrica Guthe et al. (2002); Kaufman (1998). Reconstrução visual tem sido largamente empregada em problemas onde a representação geométrica e topológica dos objetos não é de fundamental importância.

Uma abordagem não diretamente relacionada ao problema de geração de malhas a partir de imagens, mas que possui uma fundamentação teórica relevante para este contexto é o trabalho proposto por Boissonnat e Oudot (2005). Embora trate do problema de geração de superfícies a partir de nuvens de pontos, esse trabalho apresenta uma fundamentação teórica de garantia de reconstrução correta que pode ser adaptada a problemas de geração de malhas a partir de imagens. 


\subsection{Contextualização da proposta}

A partir do levantamento apresentado acima, podemos observar que métodos capazes de criar modelos apropriados para fins de simulações numéricas utilizam uma etapa de pré-processamendo na imagem de entrada. Na maioria dos casos esta etapa de préprocessamendo chega ao extremo de binarizar a imagem de entrada antes do processo de geração de malha. Por outro lado, algoritmos que não dependem muito de uma etapa de pré-processamendo visam representar a imagem de entrada utilizando a malha, sem distinguir objetos contidos na imagem.

A diferença entre os algoritmos reportados na literatura e a estratégia Imesh, proposta neste trabalho, é que a técnica Imesh assume como entrada uma imagem não pré-processada. A partir dessa imagem, o processo é dividido em três passos principais: Construção de malha, Particionamento de malha e Refinamento de qualidade. O primeiro passo, Construção de malha, visa aproximar a imagem de entrada utilizando uma malha Delaunay criada a partir de diferentes critérios de erro. O segundo passo do algoritmo, Particionamento de malha, tem como meta detectar objetos contidos na malha. Este é um processo equivalente ao processo de segmentação de imagens, porém, a malha criada apresenta um novo contexto de segmentação, com características particulares. Neste processo de particionamento são utilizados, além dos padrões de cor obtidos da imagem, critérios e relações geométricas oriundas da malha. Finalmente o terceiro passo, Refinamento de qualidade de malha, objetiva inserir critérios de qualidade na malha gerada, respeitando as fronteiras e a rotulação de simplexos definidas pelo processo de Particionamento de malha. Para este último passo utiliza-se uma adaptação do marco teórico proporcionado por algoritmos de refinamento de malha Delaunay.

Embora o Imesh adote uma abordagem iterativa semelhante as empregadas pelos métodos de modelagem de imagem, este disponibiliza um arcabouço mais flexível, possibilitando que diferentes decomposições simpliciais sejam obtidas dependendo da função de erro escolhida. De fato, a aproximação da imagem pela malha é guiada por uma função não necessariamente voltada para minimização do erro de interpolação, o que possibilita a adequação do esquema de refinamento para fins de reconstrução 3D. Mais ainda, os critérios utilizados para o processo de Construção de malha podem ser direcionados para facilitar as operações das etapas seguintes (Particionamento e Refinamento). Outra característica importante do algoritmo Imesh é que a maioria das operações utilizadas no processo podem ser 
direta e facilmente estendidas de duas para três dimensões, especialmente nas duas primeiras etapas (Construção e Particionamento). De fato, apresentaremos resultados bidimensionais (algoritmo Imesh-2) e tridimensionais (algoritmo Imesh-3). Parte dos resultados descritos neste trabalho podem se encontrados em: Cuadros-Vargas e Nonato (2004); Cuadros-Vargas et al. (2005); Cuadros-Vargas e Nonato (2006); Cuadros-Vargas et al. (2006b,a). 


\section{Capítulo}

\section{Conceitos relacionados}

Apresentamos neste capítulo os conceitos básicos e notação empregados no seguintes capítulos. Definições específicas envolvendo particularidades de cada tema serão apresentadas oportunamente. A Seção 3.1 apresenta os conceitos relacionados a Complexos Simpliciais, Triangulação de Delaunay e Diagrama de Voronoi. Seguidamente, a Seção 3.2 discute sobre algoritmos de refinamento de malhas Delaunay com critérios de qualidade. Finalmente, a Seção 3.3 descreve uma estratégia de segmentação de imagens baseada em campos aleatórios Markovianos. Todos esses conceitos são importantes pois serão utilizados durante a descrição do algoritmo Imesh (Capítulo 4) proposto nesta tese de doutorado.

\subsection{Complexos Simpliciais, Triangulação de Delaunay e Diagrama de Voronoi}

Seja $S=\left\{p_{0}, p_{1}, \ldots, p_{n}\right\}$ um conjunto de pontos em $\mathbb{R}^{d}, d=2$ ou 3 . $S$ é dito linearmente independente se os vetores $p_{i}-p_{0}, i=1, \ldots, n$ são linearmente independentes. Além disso, $S$ é dito estar em posição geral se não existe nenhum sub-espaço afim de $\mathbb{R}^{d}$ contendo $S$ e não existe nenhuma esfera de dimensão $d-1$ passando por $d+2$ pontos de $S$.

O fecho convexo de $S$, denotado conv $(S)$, é o conjunto formado por todas as combinações convexas dos pontos de $S$, isto é, $\operatorname{conv}(S)=\left\{p \in \mathbb{R}^{d} \mid p=\sum_{i=0}^{n} \lambda_{i} p_{i}, \sum_{i=0}^{n} \lambda_{i}=\right.$ $\left.1, \lambda_{i} \geq 0\right\}$. 
Dado um conjunto $V=\left\{p_{0}, \ldots, p_{m}\right\}, m \leq d$, com $m+1$ pontos linearmente independentes em $\mathbb{R}^{d}$, o $m$-simplexo $\sigma=\left[p_{0}, \ldots, p_{m}\right]$ gerado por $V$ é definido como o fecho convexo de $V$, sendo $m$ a dimensão de $\sigma$. Vale notar que em $\mathbb{R}^{d}$, o número máximo de pontos linearmente independentes é $d+1$, logo, os simplexos em $\mathbb{R}^{d}$ podem ser de dimensão $0,1, \ldots, d$. Simplexos de dimensão 0, 1, 2 e 3 são chamados vértice, aresta, triângulo e tetraedro, respectivamente. O fecho convexo de subconjuntos não vazios de $V$ também são, por definição, simplexos, chamados faces de $\sigma$. Dessa forma, um $m$-simplexo possui $2^{m+1}-2$ faces.

Um complexo simplicial $\mathcal{K}$ é uma coleção finita de simplexos satisfazendo as seguintes relações:

1. Se um simplexo $\sigma$ pertence a $\mathcal{K}$ então todas as faces de $\sigma$ também pertencem a $\mathcal{K}$.

2. Se os simplexos $\sigma$ e $\tau$ pertencem a $\mathcal{K}$ então $\sigma \cap \tau=\emptyset$ ou $\sigma \cap \tau$ é uma face de $\sigma$ e $\tau$.

A dimensão de um complexo simplicial $\mathcal{K}$ é dada pela maior dimensão dos simplexos pertencentes a $\mathcal{K}$. Se um subconjunto $\mathcal{K}^{\prime}$ de $\mathcal{K}$ é também um complexo simplicial então $\mathcal{K}^{\prime}$ é denominado um sub-complexo de $\mathcal{K}$.

A estrela de um simplexo $\sigma \in \mathcal{K}$, denotada $s t(\sigma)$, é o conjunto de todos os simplexos em $\mathcal{K}$ (incluindo suas faces) que possuem $\sigma$ como face. O laço (link) de um simplexo $\sigma$, denotado $l k(\sigma)$, é o conjunto dos simplexos em $s t(\sigma)$ que não interceptam $\sigma$.

Um complexo simplicial $\mathcal{K}$ de dimensão $m$ em $\mathbb{R}^{d}$ é uma $m$-triangulação se todo $k$ simplexo de $\mathcal{K}, k<m$, é face de algum $m$-simplexo de $\mathcal{K}$. Uma $m$-triangulação $\mathcal{T}$ é uma variedade linear por partes se o laço de todo vértice em $\mathcal{T}$ é homeomorfo a uma esfera ou semi-esfera de dimensão $m-1$. Vértices cujo laço não é homeomorfo a uma esfera ou semi-esfera é chamado de singular.

A partir dos conceitos apresentados acima, pode-se definir a Triangulação de Delaunay e o Diagrama de Voronoi, da seguinte maneira:

Seja $S \subset \mathbb{R}^{d}$ um conjunto de pontos em posição geral. A triangulação de Delaunay de $S$ é uma $d$-triangulação $\mathcal{D} \mathcal{T}$ (ver Figura 3.1 (a)), satisfazendo as seguintes condições:

1. Os vértices de $\mathcal{D} \mathcal{T}$ são os pontos de $S$.

2. A união de todos os simplexos de $\mathcal{D} \mathcal{T}$ forma o fecho convexo de $S$. 


\subsection{COMPLEXOS SIMPLICIAIS, TRIANGULAÇÃO DE DELAUNAY E DIAGRAMA DE VORONC}

3. A circunesfera (esfera circunscrita) de todo $d$-simplexo não contém pontos de $S$ em seu interior.

Pode-se mostrar que, devido a hipótese de posição geral dos pontos em $S$, existe apenas uma triangulação satisfazendo as três condições acima.

O diagrama de Voronoi é o dual geométrico da triangulação de Delaunay e pode ser definido associando a cada simplexo de dimensão $k$ em $\mathcal{D} \mathcal{T}$ uma célula ${ }^{1}$ de dimensão $d-k$ (ver Figura 3.1 (b)). Tal associação é feita da seguinte forma: Seja $\sigma \in \mathcal{D} \mathcal{T}$ um simplexo de dimensão $k \leq d$. O conjunto dos pontos de $\mathbb{R}^{d}$ que são equidistantes dos vértices de $\sigma$, e que estão mais próximos desses vértices do que de qualquer outro vértice de $\mathcal{D} \mathcal{T}$, formam a célula associada a $\sigma$. Pode-se mostrar que a união de todas as células associadas aos simplexos de $\mathcal{D} \mathcal{T}$ é uma decomposição celular de $\mathbb{R}^{d}$, denominada diagrama de Voronoi (Fortune, 1994). Em particular, as células de dimensão zero do diagrama de Voronoi (duais aos $d$-simplexos) são chamadas de vértices de Voronoi e as 1-células de arestas de Voronoi.

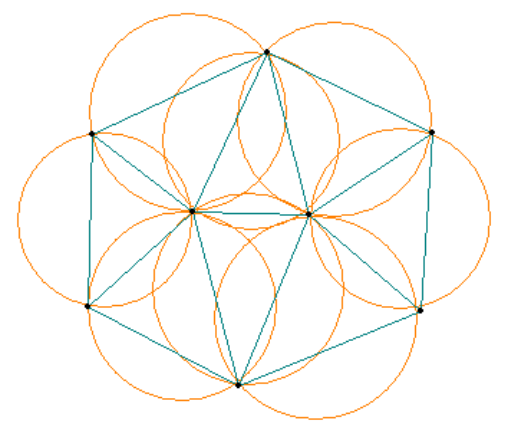

(a) Triangulação de Delaunay e circunesferas vazias.

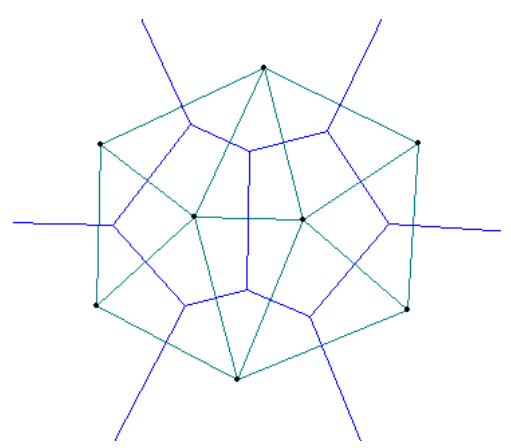

(b) Triangulação de Delaunay e seu dual Diagrama de Voronoi

Figura 3.1: Triangulação de Delaunay e Diagrama de Voronoi (Exemplo bidimensional).

Esta relação entre a triangulação de Delaunay e o diagrama de Voronoi, chamada dualidade, é explorada por vários algoritmos de reconstrução tridimensional (Boissonnat (1988); Geiger (1993b); Bajaj et al. (1996); Amenta et al. (2001); Nonato et al. (2005)).

\footnotetext{
${ }^{1}$ No contexto desta dualidade, uma célula deve ser entendida como a interseção de um número finito de semi-espaços afins de $\mathbb{R}^{d}$. A dimensão da célula é dada pela dimensão do menor subespaço afim que a contém.
} 
Por outro lado, a triangulação de Delaunay possui propriedades muito interessantes. Por exemplo, em $\mathbb{R}^{2}$, dentre todas as possíveis triangulações de um conjunto de pontos, a triangulação de Delaunay maximiza o ângulo mínimo da triangulação e minimiza o maior circuncírculo (Fortune, 1992), sendo ainda a triangulação que minimiza os limitantes de erro de interpolação linear (Shewchuk, 2002b). outras propriedades podem ser encontradas em: Fortune (1994); Aurenhammer. (1991); Edelsbrunner (2001).

Diversos algoritmos têm sido propostos para o cálculo, tanto da triangulação de $D e$ launay (Shewchuk, 2000a), quando do Diagrama de Voronoi (Aurenhammer., 1991). Tais algoritmos têm sido implementados em diversas bibliotecas de geometria computacional, sendo a biblioteca $C G A L$ (Fabri et al., 2000) uma das mais relevantes. Todas as implementações realizadas neste trabalho tiveram como base a biblioteca $C G A L$.

\subsection{Refinamento de malhas Delaunay}

Nesta seção apresentamos uma descrição de aspectos relacionados aos principais algoritmos de geração de malhas Delaunay com critérios de qualidade, em duas e três dimensões. Começaremos definindo o problema clássico de geração de malha na Seção 3.2.1. A seguir a Seção 3.2.2 apresenta alguns critérios de qualidade utilizados no caso do refinamento Delaunay bidimensional. As Seções 3.2.3 e 3.2.4 descrevem dois algoritmos clássicos de refinamento Delaunay em duas dimensões, os algoritmos de Chew e de Ruppert. A Seção 3.2.5 apresenta algumas considerações adicionais sobre algoritmos de refinamento Delaunay bidimensionais. A Seção 3.2.6 discute sobre critérios de qualidade aplicados para tetraedros. A Seção 3.2.7 descreve o algoritmo de refinamento Delaunay tridimensional criado por Shewchuk. Finalmente, a Seção 3.2.8 apresenta algumas considerações finais sobre algoritmos de refinamento Delaunay tridimensionais.

\subsubsection{O problema clássico de gerar malhas}

O problema de gerar uma malha triangular em 2D ou 3D pode ser definido de forma simplificada como: dado um grafo $G=(S, L)$, onde $S$ é um conjunto de pontos e $L$ é um conjunto de simplexos, uma malha de $G$ é uma triangulação $K$ de $S$ restrita por $L$. Isto é, o conjunto de simplexos $L$ que deve estar contido em $K$. Em geral $G$ é um conjunto de 
polígonos ou poliedros. Alguns algoritmos buscam gerar uma triangulação onde os elementos do polígono (ou poliedro) de entrada estejam contidos na união de células da malha gerada, ou seja, não é exigido que uma face do polígono seja também uma face da malha. Esse é o caso dos algoritmos de refinamento Delaunay apresentados neste trabalho. Dois aspectos importantes devem ser observados durante o processo de geração de malha: o número de simplexos gerados e a razão de aspecto desses simplexos, isto é, deve-se evitar um número excessivo de simplexos mantendo a boa qualidade dos mesmos.

\subsubsection{Qualidade de triângulos}

A qualidade, ou aspecto, de um triângulo pode ser medida utilizando diferentes critérios. Uma comparação entre as medidas de qualidade existentes na literatura pode ser encontrada em Pébay e Baker (2001). Uma medida bastante utilizada é baseada no menor ângulo do triângulo. Pode ser mostrado que o menor ângulo de um triângulo está relacionado com a razão base/altura e com a razão circunraio/menoraresta (Edelsbrunner, 2000).

Considere o triângulo $a b c$ da Figura 3.2. Repare que limitar inferiormente o menor ângulo de um triângulo implica em limitar também o maior ângulo e a razão $|b-x| /|a-c|$. O maior ângulo é no máximo $\pi-2 \theta$, onde $\theta$ é o menor ângulo, portanto, se o menor ângulo é limitado não próximo a zero, o maior ângulo também estará distante de $\pi$. Ou seja, se o menor ângulo ocorre no vértice $a$ e o maior em $b$, então temos que: $|b-x|=|b-a| \operatorname{sen}(\theta)$. Temos ainda que $|a-b| \geq|b-c|, \operatorname{logo}|b-a| \geq|c-a| / 2$, portanto temos que:

$$
\frac{1}{\sin (\theta)} \leq \frac{|c-a|}{|b-x|} \leq \frac{2}{\sin (\theta)}
$$

O que implica que um limite em $\theta$ também limita a razão: $|c-a| /|b-x|$, garantindo a boa qualidade do triângulo. Também pode ser mostrado que:

$$
\frac{\text { circumraio }}{\text { menoraresta }}=\frac{1}{2 \sin (\theta)}
$$

De fato, a Figura 3.3 mostra o desenho de uma propriedade conhecida da geometria euclidiana relacionando os ângulos $i k j$ e $i c j$ da Figura. A partir deste fato e utilizando a 


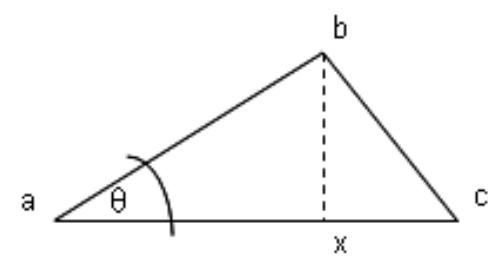

Figura 3.2: Triângulo e medidas de qualidade

propriedade dos senos tem-se:

$$
\sin (\theta)=\frac{d}{2 r}=\frac{\text { menoraresta }}{2 \text { circunraio }}
$$

logo:

$$
\frac{\text { circunraio }}{\text { menoraresta }}=\frac{r}{d}=\frac{1}{2 \sin (\theta)}
$$

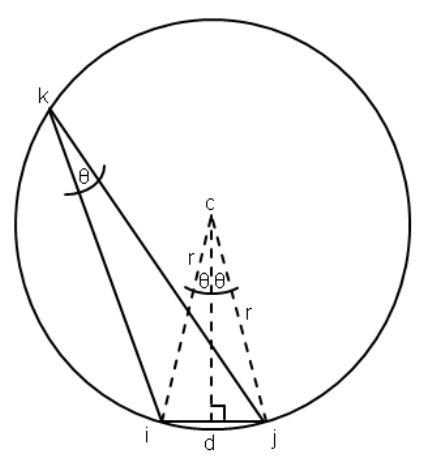

Figura 3.3: Imagem que demonstra $d=2 * r * \sin (\theta)$

Por tanto, limitando $\frac{r}{d}$ também limitamos o menor ângulo. Denominaremos à razão $\frac{r}{d}$ como o critério $\rho$, para medir a qualidade de um triângulo. Os algoritmos de refinamento Delaunay em geral buscam triangulações com boa qualidade e um número não muito grande de triângulos (Shewchuk, 1999; Edelsbrunner, 2000). Estes algoritmos procuram eliminar triângulos onde a razão $\rho$ é grande. Desta forma, garante-se que os triângulos com ângulos pequenos também serão eliminados. Esse processo é feito geralmente por meio da inclusão do circuncentro de um triângulo ruim na triangulação ${ }^{2}$.

\footnotetext{
${ }^{2}$ Existe outro tipo de ponto, que pode ser utilizado no lugar do circuncentro, chamado Off-Center (Üngör, 2004). Este tipo de centro toma como base o ponto circuncentro para calcular uma melhor posição de inserção.
} 
Aspectos importantes dos algoritmos de refinamento Delaunay 2D são:

- Um triângulo ruim $(\rho \geq B)$ é eliminado com a inserção do seu circuncentro.

- Como o circuncírculo de um triângulo ruim não contém nenhum ponto da triangulação no seu interior (exceto $p$ ), toda nova aresta tem comprimento pelo menos $r$, onde $r$ é o raio do círculo.

- Se o limite para $\rho$ é $B$, temos que para um triângulo ruim $\rho \geq B$, as novas arestas criadas com a inserção do circuncentro têm comprimento pelo menos $B d$.

- Se $B \geq 1$, o processo de inserção de pontos irá sempre terminar pois não será possível incluir arestas menores do que a menor aresta já existente na triangulação, e todos os triângulos terão o menor ângulo limitado por $\rho$.

\subsubsection{Algoritmo de Chew}

O algoritmo criado por Paul Chew (Chew, 1993; Shewchuk, 1999) gera malhas bidimensionais a partir de um polígono de entrada. Chew descreve seu algoritmo da seguinte maneira: Seja uma triangulação de Delaunay cuja menor aresta é hmin. O algoritmo introduz o circuncentro de todo triângulo cujo circunraio é maior que hmin. Observe que:

- O algoritmo nunca introduz arestas menores que $\mathrm{hmin}$.

- Todos os vértices são separados por uma distancia mínima hmin.

O problema com o algoritmo de Chew é que arestas de bordo podem impossibilitar a inclusão do circuncentro no interior do polígono de entrada. Para resolver este problema Chew propõe que as arestas do polígono de entrada sejam subdivididas em sub-segmentos de comprimento $l$ onde $h \leq l \leq \sqrt{3} h$. O valor $h$ deve ser um parâmetro definido pelo usuário de tal forma que:

- $h$ seja pequeno suficiente para possibilitar a subdivisão dos segmentos.

- $h$ seja menor que a menor distância entre dois pontos quaisquer do polígono de entrada.

Inserindo Off-Centers pode-se obter uma redução boa da quantidade de pontos no processo de refinamento Delaunay. 
A subdivisão do polígono de entrada de acordo com $h$ garante que as arestas de tais polígonos estarão na triangulação e os circuncentros sempre serão inseridos no interior do polígono de entrada.

No final do refinamento não existirá na malha triângulos com ângulos menores que $30^{\circ}$. Ou seja, supongo que o polígono de entrada não possua um ângulo pequeno entre duas arestas consecutivas, não é possível garantir que $\rho$ seja menor que uma constante préestabelecida. De fato, no final do refinamento tem-se que $\rho=r / d \leq r / h m i n \leq 1$, logo com respeito ao ângulo mínimo temos que:

$$
\frac{1}{2 \sin (\theta)} \leq 1 \Rightarrow \sin (\theta) \geq \frac{1}{2} \Rightarrow \theta \geq \arctan \left(\frac{1}{2}\right)=30^{\circ}
$$

A partir de um polígono de entrada (Figura 3.4 (a)), este algoritmo produz sempre malhas com densidades uniformes (veja Figura 3.4 (b)) e isto pode ser uma vantagem ou desvantagem segundo a finalidade do processo de geração de malha.

\subsubsection{Algoritmo de Ruppert}

O algoritmo de Jim Ruppert (Ruppert, 1995; Shewchuk, 1999) é provavelmente o primeiro algoritmo teoricamente fundamentado com resultados satisfatórios na prática. Este algoritmo é um aprimoramento do algoritmo de Chew, permitindo que a triangulação varie de densidade em diferentes regiões do domínio. O algoritmo de Ruppert possui dois passos básicos:

1. Recuperar todas as arestas do polígono de entrada que não apareçam na triangulação de Delaunay inicial. Suponha $a b$, um segmento não contido na triangulação de Delaunay dos vértices do polígono de entrada. Neste caso, o círculo diametral de $a b$ (círculo com centro em $(a+b) / 2$ e raio $|a-b| / 2)$, contém algum vértice do polígono de entrada em seu interior. Um ponto dentro do círculo diametral de um segmento do polígono de entrada é chamado ponto invasor desse segmento. Neste caso o ponto médio do segmento (centro do círculo diametral) é inserido na triangulação. Este passo é repetido enquanto existirem segmentos invadidos.

2. Suponha $a b c$ um triângulo ruim, ou seja, seu ângulo mínimo é menor que um limite inferior, então, o circuncentro deste triângulo será incluído se este circuncentro não 
invadir um segmento do polígono de entrada. No caso do circuncentro invadir um segmento, ele não é inserido e o ponto médio do segmento é adicionado à triangulação. Este passo é repetido enquanto existirem triângulos ruins.

Pode ser provado que este algoritmo termina quando o polígono de entrada não possui ângulos menores que $30^{\circ}$ entre arestas consecutivas (Shewchuk, 1999). Este algoritmo produz uma quantidade menor de triângulos que o algoritmo de Chew, descrito anteriormente (veja Figura $3.4(\mathrm{c}))$.

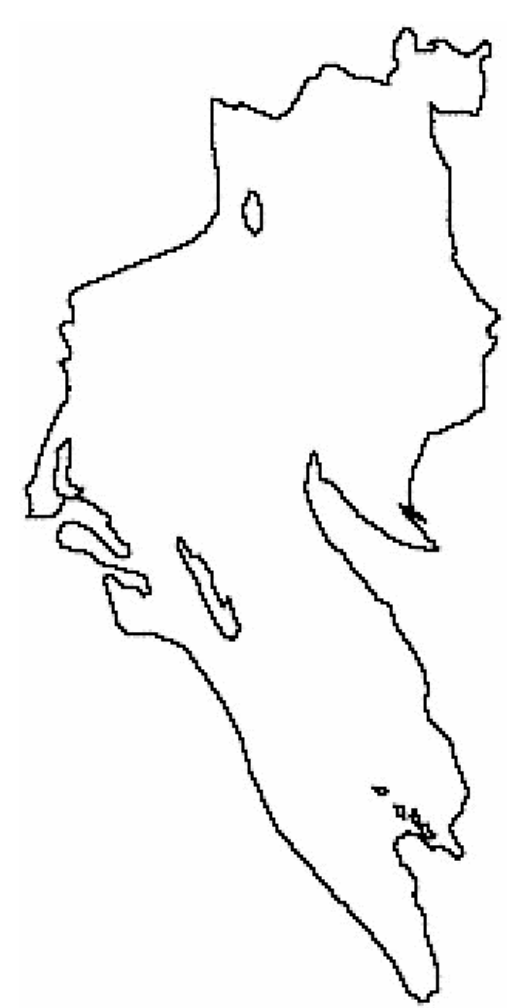

(a) Polígono de entrada que representa a Lago Superior da América do Norte

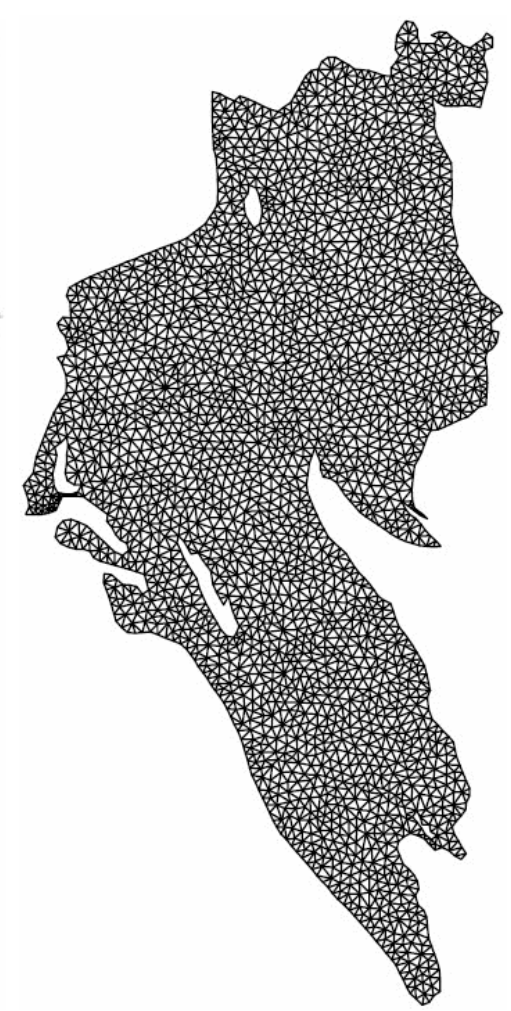

(b) Resultado do algoritmo de Chew

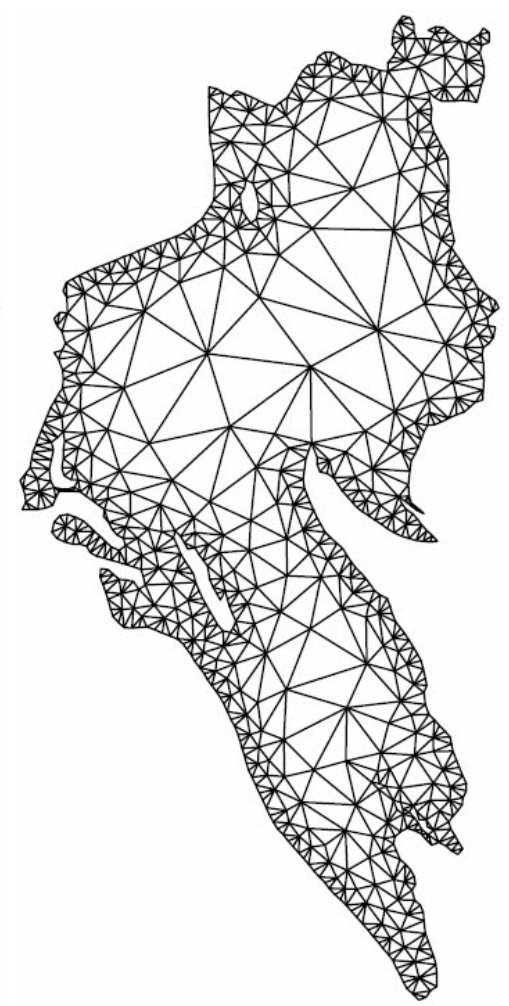

(c) Resultado do algoritmo de Ruppert

Figura 3.4: Resultados de exemplo dos algoritmos de refinamento Delaunay de Chew e Ruppert (Malhas geradas pelo Bern Eppstein Gilbert) 


\subsubsection{Considerações sobre algoritmos de refinamento Delaunay bidimensionais}

Quando no domínio de entrada, para um algoritmo de refinamento, existirem ângulos menores que $30^{\circ}$, não se tem garantia de termino para o algoritmo. Sendo assim, estes elementos devem ser tratados de forma especial. A Figura 3.5 (a) mostra um exemplo deste fato para o caso bidimensional, com um domínio de entrada que contem duas regiões $r 1$ e $r 2$, onde $r 2$ apresenta um ângulo considerado pequeno. Inicialmente o vértice $a$ viola o círculo diametral da aresta $b c$, devendo esta ser dividida inserindo-se o vértice $v_{0}$. Logo a inserção de $v_{0}$ provoca a violação da aresta $b a$ provocando a inserção de $v_{1}$. Este fenômeno se repete e uma seqüência infinita $\left(v_{0} \ldots v_{\infty}\right)$ de inserções é provocada, fazendo com que o algoritmo nunca termine. Existem na literatura algumas estratégias para tratar e isolar este tipo de problema durante o processo de refinamento.

A abordagem descrita no trabalho de Shewchuk (2002a), sugere que segmentos formando um ângulo pequeno sejam divididos em círculos concêntricos, cujos raios sejam potências de 2. Utilizando tal abordagem pode ser provado que o algoritmo termina. Esta idéia pode ser apreciada na Figura 3.5 (b).

Uma outra forma de tratar ângulos pequenos durante o refinamento é apresentada no trabalho realizado por Miller et al. (2003). Nessa abordagem, novos vértices são inseridos dois a dois também em círculos concêntricos. Neste caso, a idéia para garantir que o algoritmo termine, é fazer como que, durante o processo de refinamento, triângulos que contém ângulos pequenos ou vértices que foram inseridos em círculos concêntricos sejam mantidos. A Figura 3.5 (c), mostra uma representação desta abordagem.

Utilizando abordagens como as descritas acima, pode-se conseguir que o algoritmo termine garantindo a qualidade dos elementos da malha exceto nos triângulos próximos aos ângulos pequenos do domino de entrada.

\subsubsection{Qualidade de tetraedros}

Podemos classificar tetraedros de má qualidade em dois grupos: os que possuem vértices próximos a uma reta e os que possuem vértices próximos à um plano (Shewchuk, 2000a; Edelsbrunner, 2000). O primeiro grupo, com vértices próximos a uma aresta é chamado de tetraedros magros (skinny), sendo possível distinguir cinco tipos, como mostra a Figura 3.6 


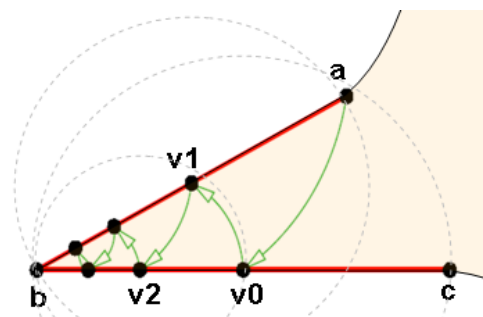

(a) O problema do refinamento infinito causado por um ângulo pequeno

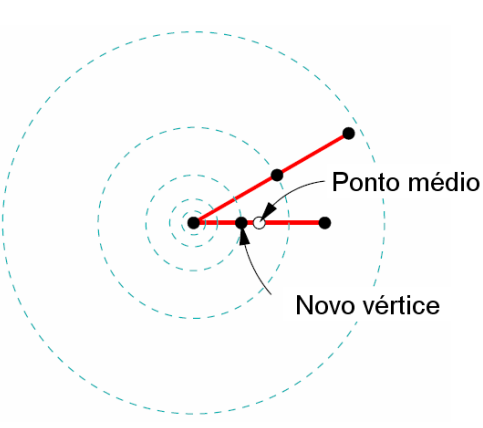

(b) Tratamento de ângulo pequeno com a abordagem de Shewchuk (2002a)

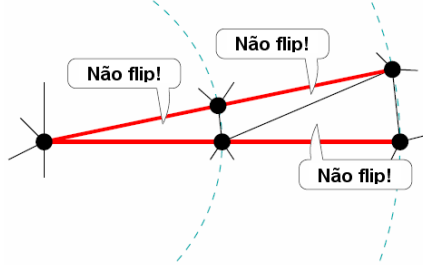

(c) Tratamento de ângulo pequeno com a abordagem de Miller et al. (2003)

Figura 3.5: O problema de ângulos pequenos e seu tratamento. (Imagens extraídas do trabalho de Shewchuk (2005))

(a). O segundo grupo, com vértices próximos a um plano, são chamados de tetraedros chatos (flat), nesse caso é posível identificar quatro tipos, como apresentado na Figura 3.6 (b).

De forma similar aos triângulos, uma medida de qualidade definida pela razão $\rho$, ou seja, $\rho=\frac{\text { circunraio }}{\text { menoraresta }}$, pode ser estabelecida. Um tetraedro minimiza a razão $\rho$ se ele for regular. Isto é, por exemplo, se as seis arestas do tetraedro têm comprimento igual a 1, então $\rho=\sqrt{6} / 4$. Observe na Figura 3.6 que um tetraedro magro tem uma área pequena e possui, ou uma aresta curta ou um grande circunraio. Da mesma forma, um tetraedro chato que não é sliver possui, ou uma aresta pequena, ou um circunraio grande. O problema com o critério de qualidade $\rho$ é que ele não é capaz de detectar tetraedros do tipo sliver, pois este possui arestas e circunraio de tamanho similar. Embora o critério da razão $\rho$ não reconheça tetraedros sliver, ele introduz outras boas qualidades no processo de refinamento de uma malha. Por exemplo, esta medida é uma extensão direta de duas para três dimensões. Este fato é particularmente importante no contexto do algoritmo Imesh (Capítulo 4) proposto neste trabalho, pois uma das características importantes deste algoritmo é que muitos dos passos utilizados não dependem da dimensão. Outras medidas de qualidade bastante investigadas na literatura são o critério do ângulo sólido e o critério da razão entre raios (A. Liu, 1994). Utilizando tais critérios é possível detectar tetraedros ruins do tipo sliver. 

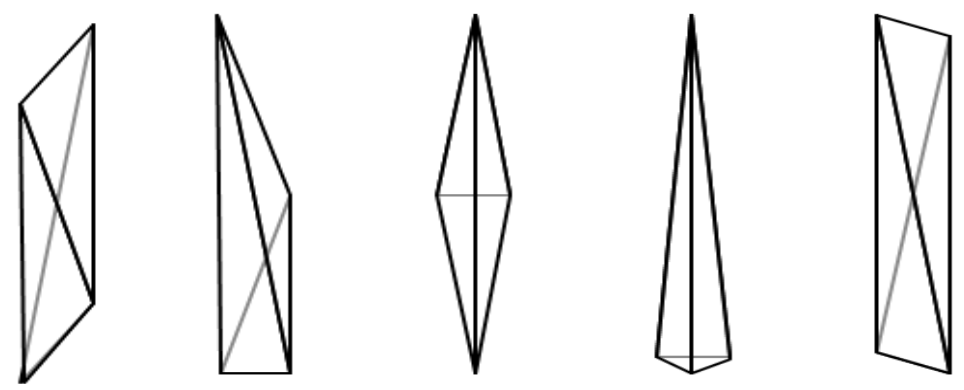

(a) Tetraedros magros (skinny).
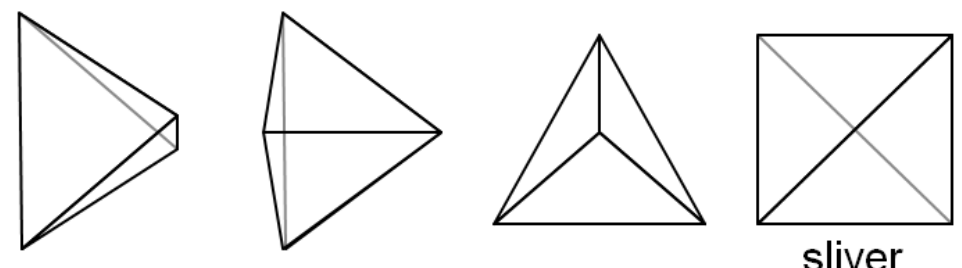

sliver

(b) Tetraedros chatos (flat).

Figura 3.6: Tetraedros considerados ruins.

\subsubsection{Algoritmo de Shewchuk}

Este algoritmo foi criado no trabalho de Shewchuk (1997) e tem a mesma idéia do algoritmo de Ruppert estendida para três dimensões. Shewchuk define o algoritmo como segue: Seja P um poliedro onde o ângulo entre dois de seus segmentos, ou entre um segmento e uma face, ou entre duas faces não é menor que $\pi / 2$. O objetivo do refinamento 3D é construir uma triangulação de Delaunay $\mathcal{D} \mathcal{T}$ que contenha $P$ (possivelmente subdividido) e possua a propriedade da razão $\rho$. Subdividir $P$ significa que toda face de $P$ pode ser recuperada pela união de faces em $\mathcal{D} \mathcal{T}$. O mesmo vale para as arestas, ou seja, uma aresta de $P$ pode ser obtida pela união de arestas de $\mathcal{D} \mathcal{T}$. Desta forma, uma aresta de $P$ é constituída de sub-segmentos com vértices em $\mathcal{D} \mathcal{T}$ e uma face é constituída de sub-faces (triângulos) com vértices e arestas em $\mathcal{D} \mathcal{T}$.

O algoritmo de refinamento insere vértices de acordo com 3 regras:

1. Se um segmento é invadido, ele é dividido inserindo-se o ponto médio deste segmento 
como novo vértice na triangulação de Delaunay. Um vértice invade o segmento se a esfera diametral do segmento possui o vértice em seu interior. Um vértice invade uma face se a esfera equatorial da face possui o vértice em seu interior. Os novos segmentos criados podem ou não ser invadidos. A sub-divisão continua até que todos os segmentos sejam não invadidos.

2. Se uma sub-face de $P$ é invadida então o circuncentro de sua esfera equatorial é inserida na triangulação de Delaunay. Se o circuncentro invade um ou mais sub-segmentos então ele não é inserido e os segmentos invadidos são sub-divididos como na regra 1.

3. Se um tetraedro dentro de $P$ não têm a propriedade da razão $\rho$, então o circuncentro deste tetraedro é adicionado na triangulação de Delaunay. Se tal circuncentro invade uma face ou segmento, então ele não é adicionado e as faces e segmentos invadidos são subdivididos como nas regras 1 e 2 .

A primeira regra tem prioridade sobre as demais. A segunda tem prioridade sobre a terceira. Segmentos invadidos podem ou não estar na triangulação de Delaunay, isto é, se um segmento do poliedro de entrada não aparece na triangulação então ele certamente está invadido. Após a recuperação dos segmentos e faces pelas regras 1 e 2, os tetraedros externos devem ser eliminados. Rotulando os vértices inseridos como 1,2 ou 3 de acordo com a regra que os inseriu temos: As regras 1 e 2 garantem que não existem segmentos e faces invadidos. Este fato também garante que o circuncentro de todo tetraedro está no interior da triangulação após a eliminação dos tetraedros externos.

Uma das formas para verificar as faces de $P$ em $\mathcal{D} \mathcal{T}$ é manter uma triangulação 2D para cada face de $P$ e verificar que as triangulações das faces em $2 \mathrm{D}$ e $3 \mathrm{D}$ são as mesmas. Caso não sejam, o circuncentro dos triângulos inexistentes são inseridos. No final deste algoritmo temos uma malha bem comportada e com tetraedros com uma razão $\rho \geq 2$. No entanto, esta malha ainda pode conter tetraedros do tipo sliver que devem ser tratados.

\subsubsection{Considerações sobre algoritmos de refinamento Delaunay tridimensionais}

Segundo Shewchuk, um tetraedro sliver pode sempre ser eliminado inserindo um vértice no seu circuncentro, mas não se tem uma garantia clara de que o refinamento termine. 


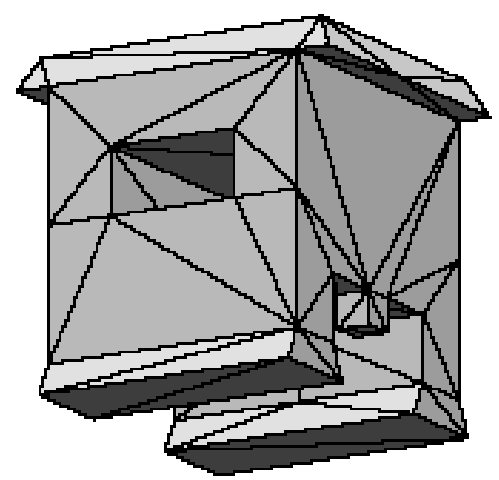

(a) Malha de entrada de exemplo.

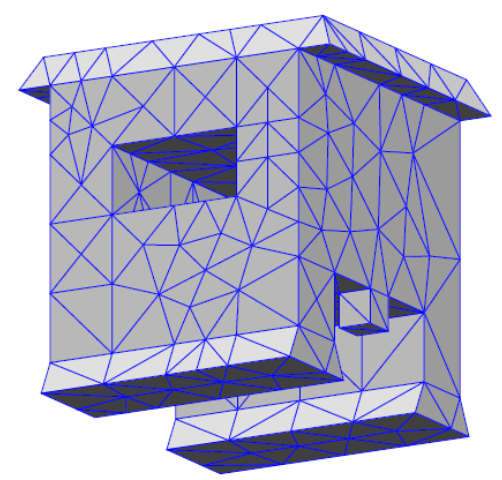

(b) Refinamento com $B=$ $1.2, \quad 334$ vértices, 1009 tetraedros.

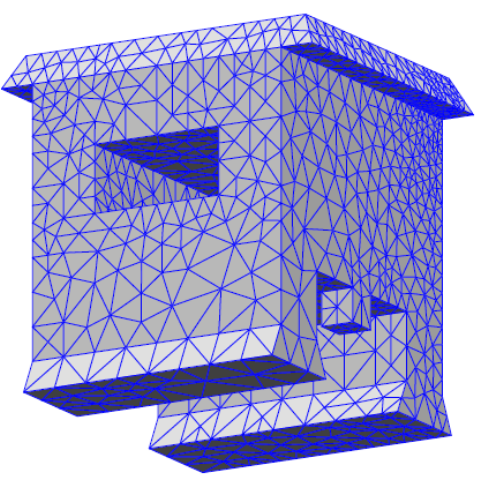

(c) Refinamento com $B=$ 1.041, 3144 vértices, 13969 tetraedros.

Figura 3.7: Resultados de exemplo do algoritmo de refinamento de Shewchuk (Imagens extraídas do trabalho de Shewchuk (2005).

Outras técnicas para o tratamento de tetraedros sliver podem ser encontradas nos trabalhos de Cheng et al. (2000); Shewchuk (1998); Chew (1997); Edelsbrunner e Guoy (2001).

Por outro lado, quando o poliedro de entrada possui ângulos pequenos, a tarefa de refinamento Delaunay se torna mais complexa. Por exemplo, a inserção de um vértice em uma face do poliedro pode eliminar outras faces adjacentes que formam parte do bordo. Este fato pode ocasionar que o algoritmo não termine. Técnicas para tratar ângulos pequenos durante o refinamento Delaunay podem ser encontradas nos trabalhos de Shewchuk (2000b); Cheng et al. (2004); Pav e Walkington (2004).

\subsection{Estratégia de segmentação EM/MPM}

Abordagens de segmentação de imagens baseadas em campos aleatórios Markovianos (MRF-Markov random field) objetivam rotular cada elemento da imagem considerando a dependência desse elemento com relação a seus vizinhos. Essa dependência é expressa em termos de uma probabilidade condicional calculada em cada elemento, sendo que, qualquer decomposição espacial que possua relação de vizinhança bem definida pode ser segmentada via $\mathrm{MRF}$, como é o caso de triangulações.

A estratégia EM/MPM para segmentação de imagens, proposta originalmente por 
Comer e Delp (2000), é constituída de dois procedimentos principais: estimativa de parâmetros (EM - Expectation Maximization) e otimização estatística (MPM - Maximizer of the Posterior Marginals), os quais podem ser descritos como segue.

Sejam $\mathbf{X}=\left(X_{1}, X_{2}, \ldots, X_{N}\right)$ e $\mathbf{Y}=\left(Y_{1}, Y_{2}, \ldots, Y_{N}\right)$ um campo de rótulos e a imagem observada, respectivamente, sendo $N$ o número total de elementos (pixels ou voxels) na imagem. Um elemento $X_{i}, i \in D$ de $\mathbf{X}$, onde $D$ é o domínio da imagem, é considerado uma variável aleatória, o mesmo valendo para os elementos $Y_{j}$ de $\mathbf{Y}$. Realizações amostrais de $\mathbf{X}$ e $\mathbf{Y}$ são denotadas por $\mathbf{x}=\left(x_{1}, x_{2}, \ldots, x_{N}\right)$ e $\mathbf{y}=\left(y_{1}, y_{2}, \ldots, y_{N}\right)$.

O campo de rótulos $\mathbf{X}$ é assumido como um campo aleatório de Markov, logo sua função densidade de probabilidade pode ser descrita como

$$
p_{\mathbf{X}}=\frac{1}{z} \exp \left(-\sum_{(i, j) \in \mathcal{C}} \alpha t\left(x_{i}, x_{j}\right)\right)
$$

onde $\mathcal{C}$ é o conjunto de todos os pares de elementos vizinhos (em geral chamados de cliques) e $\alpha$ é um parâmetro que define o peso da função $t$. A função $t$, chamada de função penalidade, é definida como:

$$
t\left(x_{i}, x_{j}\right)= \begin{cases}0 & \text { se } x_{i}=x_{j} \\ 1 & \text { se } x_{i} \neq x_{j}\end{cases}
$$

Nota-se, a partir da definição da função $t$ (3.7), que elementos vizinhos com rótulos diferentes penalizam a probabilidade de uma dada configuração de rótulos. O peso dessa penalidade é dado pelo parâmetro $\alpha$.

A estratégia EM/MPM assume que as variáveis aleatórias $Y_{i}$ são condicionalmente independentes dado o campo de rótulos $\mathbf{X}$. Além disso, é assumido que a função densidade de probabilidade condicional de $Y_{i}$, dado $\mathbf{X}$, depende somente do valor assumido em $X_{i}$. Assume-se ainda que as variáveis aleatórias em $\mathbf{Y}$ que caracterizam um determinado rótulo $r=1, \ldots, k$ ( $k$ sendo o número de rótulos ou regiões a serem segmentadas) são gaussianas independentes e identicamente distribuídas com média $\mu_{r}$ e variância $\delta_{r}^{2}$. O conjunto de médias e variâncias $\mu_{r}$ e $\delta_{r}^{2}$ associados aos elementos da imagem que caracterizam o rótulo $r$ constituem o vetor de parâmetros $\theta=\left(\mu_{1}, \delta_{1}^{2}, \mu_{2}, \delta_{2}^{2}, \ldots, \mu_{k}, \delta_{k}^{2}\right)$ que deve ser estimado no procedimento EM.

Com as hipóteses acima e assumindo $\theta$ conhecido, a função densidade de probabilidade 
condicional de $\mathbf{Y}$ dado $\mathbf{X}$ é descrita como:

$$
f_{\mathbf{Y} \mid \mathbf{X}}(\mathbf{y} \mid \mathbf{x}, \theta)=\prod_{s=1}^{N} \frac{1}{\sqrt{2 \pi \delta_{x_{i}}^{2}}} \exp \left(-\frac{\left(y_{i}-\mu_{x_{i}}\right)^{2}}{2 \delta_{x_{i}}^{2}}\right) .
$$

Pelo teorema de Bayes, pode-se escrever

$$
p_{\mathbf{X} \mid \mathbf{Y}}(\mathbf{x} \mid \mathbf{y}, \theta)=\frac{f_{\mathbf{Y} \mid \mathbf{X}}(\mathbf{y} \mid \mathbf{x}, \theta) p_{\mathbf{X}}(\mathbf{x})}{f_{\mathbf{Y}}(\mathbf{y} \mid \theta)}
$$

Como $f_{\mathbf{Y}}(\mathbf{y} \mid \theta)$ não depende de $\mathbf{x}$ este termo não é considerado na otimização. Na verdade, o procedimento MPM procura encontrar, para cada elemento $i$, o valor de rótulo $r$ que maximiza

$$
P\left(X_{i}=r \mid \mathbf{Y}=\mathbf{y}\right)=p_{X_{i} \mid \mathbf{Y}}(r \mid \mathbf{y}, \theta)=\sum_{\mathbf{x} \in \Omega_{r, i}} p_{\mathbf{X} \mid \mathbf{Y}}(\mathbf{x} \mid \mathbf{y}, \theta)
$$

onde $\Omega_{r, i}=\left\{\mathbf{x} \mid x_{i}=r\right\}$.

Note que o cálculo exato da probabilidade marginal (3.10) é computacionalmente inviável, pois considera o conjunto de todas as possíveis configurações de rótulos para os elementos da imagem quando um dado elemento $i$ assume o rótulo $r$.

A fim de viabilizar o cálculo acima, Marroquin et al. (1987) propuseram um esquema para aproximar $P\left(X_{i}=r \mid \mathbf{Y}=\mathbf{y}\right)$ em cada elemento $i=1, \ldots, N$ e para cada rótulo $r=1, \ldots, k$. A idéia é usar um amostrador de Gibbs (German e German (1984)) para gerar uma cadeia de Markov discreta $\mathbf{X}(t)$ que converge em distribuição para $p_{\mathbf{X} \mid \mathbf{Y}}(\mathbf{x} \mid \mathbf{y}, \theta)$, dada na Equação ??. As probabilidades condicionais marginais $p_{X_{i} \mid \mathbf{Y}}(r \mid \mathbf{y}, \theta)$ que devem ser maximizadas são aproximadas como a fração de tempo que a cadeia de Markov permanece no estado $r$ para o elemento $i$, para cada $r$ e $i$. Dessa forma, se $T_{i}$ é o número de vezes que o amostrador de Gibbs visita o elemento $i$, então

$$
p_{X_{i} \mid \mathbf{Y}}(r \mid \mathbf{y}, \theta) \approx \frac{1}{T_{i}} \sum_{t=1}^{T_{i}} u_{r, i}(t)
$$

onde a função $u_{r, i}(t)$ é dada por

$$
u_{r, i}(t)= \begin{cases}1 & \text { se } X_{i}(t)=r \\ 0 & \text { se } X_{i}(t) \neq r\end{cases}
$$


Uma vez estimadas as probabilidades marginais no passo $n$, a etapa EM calcula os novos parâmetros como:

$$
\begin{gathered}
\mu_{r}(n+1)=\frac{1}{N_{r}(n+1)} \sum_{i=1}^{N} y_{i} p_{X_{i} \mid \mathbf{Y}}(r \mid \mathbf{y}, \theta(n)) \\
\delta_{r}^{2}(n+1)=\frac{1}{N_{r}(n+1)} \sum_{i=1}^{N}\left(y_{i}-\mu_{r}(n+1)\right)^{2} p_{X_{i} \mid \mathbf{Y}}(r \mid \mathbf{y}, \theta(n))
\end{gathered}
$$

onde

$$
N_{r}(n+1)=\sum_{i=1}^{N} p_{X_{i} \mid \mathbf{Y}}(r \mid \mathbf{y}, \theta(n)) \quad \forall r=1, \ldots, k
$$




\section{Capítulo}

\section{O algoritmo Imesh}

Este capítulo descreve o algoritmo Imesh criado neste projeto de doutorado. O algoritmo foi concebido para criar malhas diretamente a partir de imagens de entrada não pré-processadas. Os conceitos deste algoritmo podem ser aplicados para os casos de imagens de entrada bidimensionais ou tridimensionais, produzindo malhas triangulares e tetraedrais respectivamente. Embora nosso foco de interesse seja o caso tridimensional, resultados interessantes foram obtidos na modelagem de objetos contidos em imagens bidimensionais. Assim, neste projeto foram criadas duas versões do algoritmo: Imesh-2 para o caso bidimensional e Imesh-3 para o caso tridimensional. Embora estes algoritmos possuam diferenças substanciais devido à topologia das estruturas de dados utilizadas e situações geométricas dependentes da dimensão, as idéias principais envolvidas podem ser descritas de forma unificada. Desta forma, sempre que possível, o algoritmo será descrito utilizando termos genéricos para duas e três dimensões. Utilizaremos, durante a descrição do algoritmo, uma nomenclatura especial para expressar idéias em duas e três dimensões. A palavra célula ${ }_{d}$ será utilizada para indicar triângulo ou tetraedro. O conceito face ${ }_{d}$ para se referir à aresta de interseção entre dois

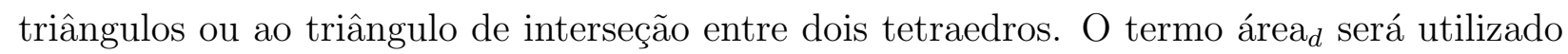
para expressar área ou volume. A expressão pixel $_{d}$ para se referir aos elementos da imagem pixels ou voxels. Porém, exemplos bidimensionais serão utilizados durante a explicação do algoritmo para facilitar o entendimento dos conceitos envolvidos. Exemplos mais complexos, e em três dimensões, serão apresentados no Capítulo 5 de resultados. 
A idéia fundamental do algoritmo Imesh, é dividir o processo em três passos principais:

- Construção de malha - Este primeiro passo, descrito na Seção 4.1, começa a partir de uma imagem de entrada não pré-processada. O objetivo deste processo é representar a imagem de entrada com uma malha Delaunay. Mais especificamente, a imagem e representada utilizando padrões de cor calculados a partir dos pixeis ${ }_{d}$ contidos dentro de cada célula ${ }_{d}$ da malha. Diferentemente das abordagens convencionais, descritas no Capítulo 2, este processo introduz uma maior flexibilidade na escolha da função de erro utilizada para aproximar a malha à imagem. Este fato possibilita a geração de modelos que podem ser adequados para um tipo de aplicação específica, como por exemplo simulação numérica ou modelagem de imagem via triangulações. Outro fator importante é que o cálculo de erro utilizado pode ser naturalmente estendido de duas para três dimensões.

- Particionamento de malha - Este segundo passo do algoritmo, descrito na Seção 4.2, visa decompor a malha gerada pelo processo anterior, possibilitando distinguir diferentes estruturas contidas na malha. O problema tratado neste processo é semelhante ao problema de segmentação de imagens. Porém, a decomposição simplicial apresenta um novo contexto de segmentação. Por exemplo, as células $d$ da malha não são regulares, e seu número é muito inferior ao número de pixeis $_{d}$ contidos na imagem. Esta diminuição de número de elementos é especialmente importante para o caso de particionamento tridimensional. Além disso, a malha disponibiliza informações adicionais que podem ser utilizadas no particionamento, possibilitando assim, resultados mais sofisticados. Por outro lado, o fenômeno do ruído numa imagem, originado pela discretização durante o processo de aquisição da imagem, acontece também ao aproximar uma imagem com uma malha. Este fenômeno é refletido nos padrões de cor que a malha utiliza para representar a imagem, e deve ser considerado nas operações de particionamento.

- Melhoramento de qualidade de malha - Este último passo é utilizado para inserir critérios de qualidade na malha particionada pelo processo anterior. Para isto, utiliza-se idéias de algoritmos de refinamento de qualidade Delaunay. A idéia básica

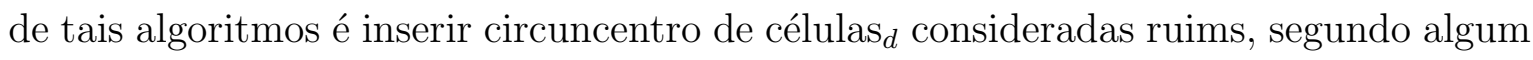
critério. Este processo deve garantir que as fronteiras definidas pelo processo de parti- 
cionamento serão mantidas no final do refinamento. Este processo também deve manter

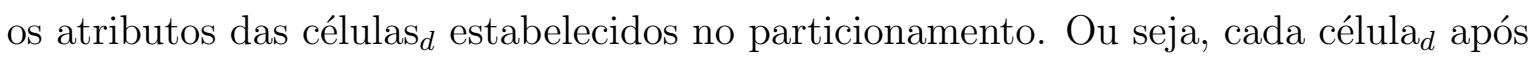
a segmentação pertence a uma sub-malha; assim, se um novo ponto é inserido na tri-

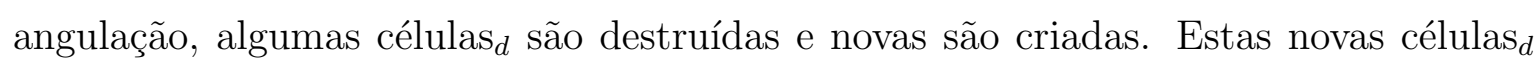
devem ser então re-identificadas para manter o particionamento coerente.

Os detalhes de como as idéias acima são implementadas são discutidos a seguir.

\subsection{Construção de malha}

No contexto deste trabalho, uma imagem é considerada como sendo uma função $I$ definida em um domínio $D \subset \mathbb{N}^{d}$ da forma $D=\left[0, \ldots, N_{1}\right] \times \ldots \times\left[0, \ldots, N_{d}\right], d \in\{2,3\}$, onde $\mathbb{N}$ é o conjunto dos números naturais. A função $I$ atribui a cada elemento do domínio $D$ um número real positivo, isto é, $I: D \rightarrow \mathbb{R}^{+}$. Cada par $(p, I(p)) \in D \times \mathbb{R}^{+}$é denominado pixel ou voxel, dependendo se $D \subset \mathbb{N}^{2}$ ou $D \subset \mathbb{N}^{3}$.

Dada uma imagem $I: D \rightarrow \mathbb{R}^{+}$, a etapa de construção da malha busca selecionar um conjunto de pontos $S \subset D$ tal que as células ${ }_{d}$ contidas na triangulação de Delaunay $\mathcal{D} \mathcal{T}$ de $S$ capturem, de alguma forma, características da imagem. Por exemplo, pode-se desejar que as células ${ }_{d}$ em $\mathcal{D} \mathcal{T}$ estejam contidas em regiões de $D$ onde $I$ não varia bruscamente.

Seja $I: D \rightarrow \mathbb{R}^{+}$uma imagem e $\mathcal{D} \mathcal{T}$ a triangulação de Delaunay gerada a partir de um conjunto de pontos $S_{0}$ na fronteira de $D$, ou seja, cada ponto em $S_{0}$ é da forma $p=\left(p_{1}, \ldots, p_{d}\right), d \in\{2,3\}$, onde pelo menos uma das coordenadas $p_{j}$ seja igual a zero ou igual a $N_{j}$, para algum $j=1, \ldots, d$.

Considere $T$ o conjunto de células ${ }_{d}$ em $\mathcal{D} \mathcal{T}$ e $E: T \rightarrow \mathbb{R}^{+}$uma função que associa, a cada célula $\sigma \in T$, uma medida de erro. O objetivo é que a função $E$ meça se uma dada célula $_{d} \sigma$ se ajusta a uma dada propriedade, possibilitando decidir se $\sigma$ deve ou não permanecer na triangulação.

Como descrito no Capítulo 2, técnicas de modelagem de imagem, em geral, definem a função $E$ como uma medida de erro de interpolação (Garland e Heckbert, 1995). A maioria destes métodos avalia a função $E$ percorrendo, para cada célula $d \sigma$, os elementos da im-

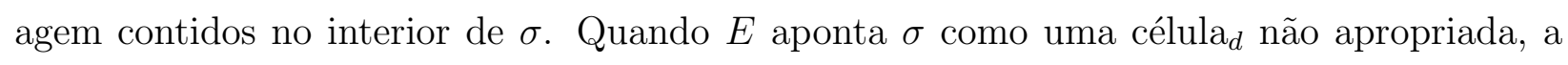
triangulação é atualizada de modo a eliminar $\sigma$. Tal atualização é geralmente conduzida 
por meio de inserção de novos vértices. Embora largamente empregada, tal estratégia apresenta dois principais inconvenientes. O caminhamento pelo interior das células ${ }_{d}$ pode exigir um grande esforço computacional, pois a cada atualização da triangulação, todas as novas

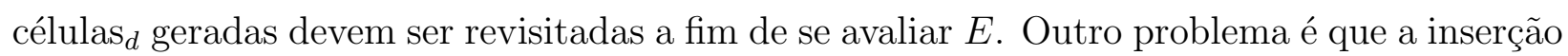
de novos vértices pode provocar um acúmulo de pontos em regiões particulares do domínio, introduzindo um número excessivo de elementos na triangulação.

Visando evitar os problemas mencionados acima, definimos uma função erro $E$

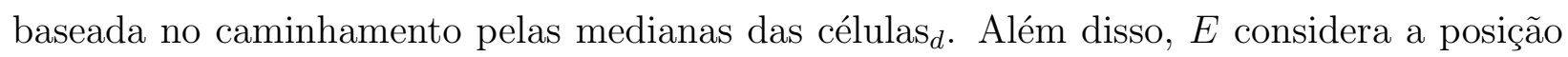
dos vértices já existentes na triangulação, procurando evitar o acúmulo de pontos. Dessa forma, a função erro proposta reduz o esforço computacional, produzindo ainda triangulações com boas propriedades.

Mais especificamente, seja $h_{0}, \ldots, h_{d}$ as $d+1$ medianas de uma célula $\sigma$. Considere o conjunto de pontos $P^{h_{j}}=\left\{p \in h_{j} \mid \mathscr{E}(p) \geq c_{E}\right\}$, onde $\mathscr{E}$ é uma medida de erro pontual em $p$ e $c_{E}$ é um escalar positivo. O conjunto $P^{h_{j}}$ contém os pontos sobre a medianas $h_{j}$ cujo erro pontual $\mathscr{E}$ é maior que um valor desejado, definido por $c_{E}$.

Denotamos por $d_{\mathcal{D} \mathcal{T}}(p)$ o quadrado da distância de $p$ ao vértice mais próximo em $\mathcal{D} \mathcal{T}$, isto é, $d_{\mathcal{D} \mathcal{T}}(p)=\min _{v_{s} \in \mathcal{D} \mathcal{T}}\left\{d_{e}^{2}\left(p, v_{s}\right)\right\}$, onde $d_{e}(\cdot, \cdot)$ é a distância euclidiana e $v_{s}$ um vértice de $\mathcal{D} \mathcal{T}$, seja $p_{h_{j}}$ o ponto de $P^{h_{j}}$ onde $d_{\mathcal{D} \mathcal{T}}$ é máxima. A partir das definições acima, definimos a função erro $E$ como:

$$
E(\sigma)= \begin{cases}\max _{j}\left\{\mathscr{E}\left(p_{h_{j}}\right)\right\} & \text { se } P^{h_{j}} \neq \emptyset \text { para algum } j=0, \ldots, d \\ 0 & \text { caso contrário }\end{cases}
$$

Desta forma, a função $E$ considera, dentre os pontos cujo erro pontual está acima de um limiar, o mais distante, em cada mediana, dos vértices da triangulação. Entre os mais distantes, $E$ assume o valor do maior erro pontual. O ponto $p_{h_{j}}$ escolhido, isto é, o ponto tal que $E(\sigma)=\mathscr{E}\left(p_{h_{j}}\right)$, é inserido na triangulação.

As duas operações principais da função erro $E$, isto é, o cálculo do erro pontual $\mathscr{E}$ e do ponto mais distante, são detalhadas a seguir. 


\subsubsection{Cálculo do erro pontual}

Existem várias possibilidades para a definição da função erro pontual, sendo que diferentes definições de $\mathscr{E}$ originam diferentes conjuntos de pontos e portanto, diferentes triangulações. Apresentamos a seguir três definições distintas para a função $\mathscr{E}$, sendo cada uma delas mais apropriada para um determinado tipo de aplicação.

\section{- $\mathscr{E}$ como erro de interpolação linear}

Uma forma imediata de definir a função de erro pontual é considerar o erro de interpolação linear. Formalmente, considere $\sigma=\left[v_{0}, \ldots, v_{d}\right]$ um célula ${ }_{d}$ e $\alpha_{i}, i=0, \ldots, d$ as coordenadas baricêntricas de um ponto $p \in h_{j}$, sendo $h_{j}$ uma das medianas de $\sigma$. Seja $\tilde{p}=\alpha_{0} I\left(v_{0}\right)+\cdots+\alpha_{d} I\left(v_{d}\right)$, a interpolação em $p$ dos valores de $I$ nos vértices de $\sigma$. Assim, pode-se definir:

$$
\mathscr{E}_{I}(p)=|\tilde{p}-I(p)|
$$

Utilizando como erro pontual $\mathscr{E}_{I}(p)$, a função erro $\mathscr{E}$ busca localizar células ${ }_{d}$ em regiões onde a aproximação linear por partes de $I$ não é boa, considerando ainda a densidade de vértices.

\section{- $\mathscr{E}$ como medida de homogeneidade}

Quando o objetivo é criar malhas apropriadas para distinguir objetos nelas, ou seja, para serem segmentadas, deve-se procurar garantir que as células $d$ da triangulação estejam contidas em regiões homogêneas da imagem. Isto é, uma mesma célula não deve atravessar objetos distintos da imagem. Tal característica é difícil de ser obtida usando a função de erro pontual $\mathscr{E}_{I}(p)$, pois a definição de $\mathscr{E}_{I}(p)$ não assegura tal propriedade.

A fim de obtermos uma triangulação com o comportamento acima, isto é, onde as células $_{d}$ evitam cruzar regiões homogêneas da imagem, definimos uma função de erro pontual que procura novos vértices em regiões de fronteira entre objetos da imagem. Tal função pode ser declarada como segue: 
Seja $A(p)=\min _{i}\left\{\alpha_{i}\right\}, i=0, \ldots, d$, uma função que associa a cada ponto $p$, das medianas de uma célula $_{d} \sigma$, a área $d$ da sua menor coordenada baricêntrica ${ }^{1}$. Considere $H(p)$ uma função binária capaz de determinar se um ponto $p$ corresponde, ou não, a uma coordenada considerada de bordo na imagem $I$. Podemos definir a função $H(p)$ de duas formas distintas, como segue:

\section{- Como um filtro que detecta altas frequências em uma imagem}

Seja $G(p)$ um detector de bordo, como por exemplo, Sobel, Prewitt, Roberts, ou Canny (Zuniga e Haralick, 1987; Jain, 1989), definimos

$$
H_{g}(p)= \begin{cases}0 & \text { se } G(p)<c_{g} \\ 1 & \text { se } G(p) \geq c_{g}\end{cases}
$$

onde $c_{g}$ é um limiar que representa os valores de alta freqüência a serem considerados.

- Como uma função que define fronteiras baseadas em um conjunto de iso-valores

Seja $\Psi=\left\{v_{0}, v_{1}, \ldots, v_{n}\right\}$, um conjunto de iso-valores que definem o bordo de regiões contidas na imagem na imagem $I$. Podemos definir então:

$$
H_{v}(p)= \begin{cases}1 & \text { se } I(p) \in \Psi \\ 1 & \text { se } \left.\exists v_{i} \in \Psi, v_{i} \in\right] I(p), I\left(p_{\text {ant }}\right)[ \\ 0 & \text { caso contrario }\end{cases}
$$

onde $p_{\text {ant }}$ representa o ponto anterior ${ }^{2}$ ao ponto $p$ durante o caminhamento sobre uma mediana de uma célula $\sigma$.

A partir das definições acima, podemos estabelecer as medidas de homogeneidade $\mathscr{E}_{g}(p)$ e $\mathscr{E}_{v}(p)$, baseadas nos conceitos de altas freqüências e conjunto de iso-valores respectivamente, da seguinte maneira:

$$
\mathscr{E}_{g}(p)=A(p) H_{g}(p)
$$

\footnotetext{
${ }^{1}$ Note que as coordenadas baricêntricas de $p$ estão relacionadas com as áreas ${ }_{d}$ das sub-células formadas por $p$ e as faces $d$ de $\sigma$.

${ }^{2}$ Observe que $p_{\text {ant }}$ somente pode ser considerado a partir da segunda coordenada no percurso de um segmento de reta ou mediana
} 


$$
\mathscr{E}_{v}(p)=A(p) H_{v}(p)
$$

As funções $\mathscr{E}_{g}$ e $\mathscr{E}_{v}$ calculam, para cada ponto considerado de bordo sobre as medianas,

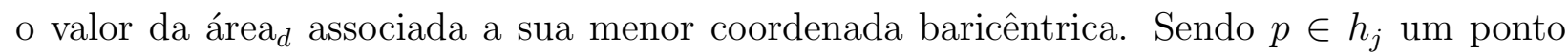
supostamente sobre o bordo de objetos da imagem, as funções $\mathscr{E}_{g}$ e $\mathscr{E}_{v}$, de fato, fornecem uma medida de quanto a célula $\sigma$ invade um objeto da imagem, ou seja, valores pequenos de $\mathscr{E}_{g}$ ou $\mathscr{E}_{v}$ significam que $h_{j}$ intercepta o bordo de um objeto da imagem próximo a uma

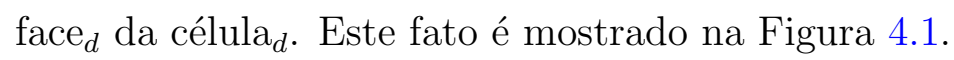

Conseqüentemente, quando $\mathscr{E}_{g}$ e $\mathscr{E}_{v}$ são utilizadas, a função $E$, na verdade, estará medindo se uma dada célula ${ }_{d} \sigma$ está ou não bem "encaixada" em uma região da imagem. Assim, valores pequenos de $E$ indicam células $d$ bem posicionados em uma região.
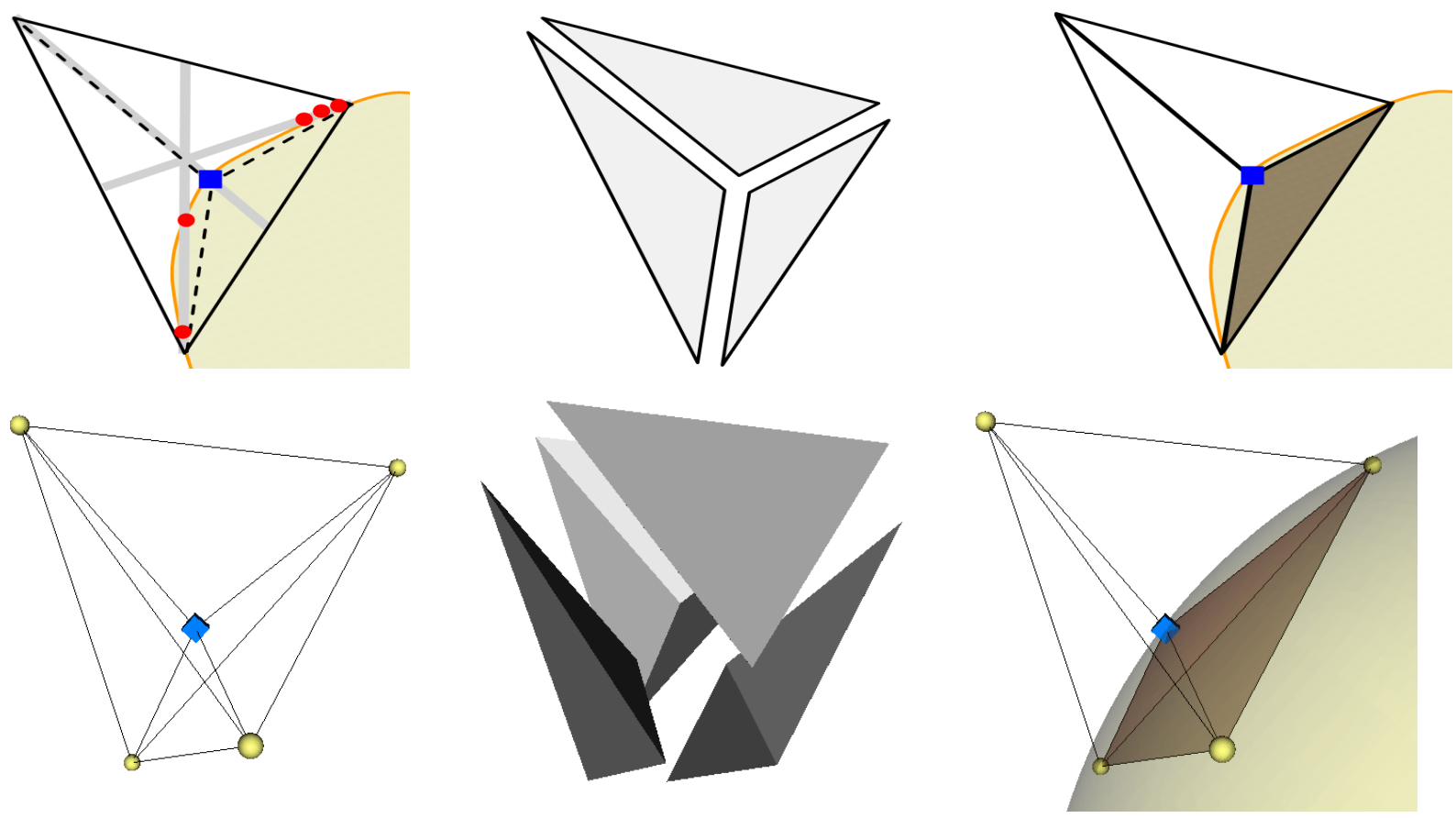

Figura 4.1: Medida de erro pontual de homogeneidade. No exemplo bidimensional (fila de cima), os círculos representam o conjunto $P^{h_{j}}$ de pontos selecionados sobre as medianas de

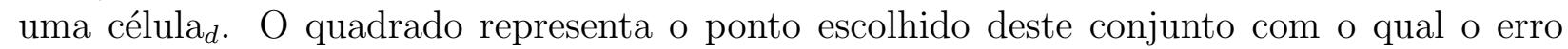
da célula ${ }_{d}$ é calculado. Logo, a idéia é estendida diretamente para o exemplo 3D (fila de abaixo). 


\subsubsection{Cálculo do ponto mais distante dos vértices da triangulação}

O custo computacional envolvido no cálculo dos pontos $p_{h_{j}}$ pode inviabilizar o uso prático do Imesh. Por outro lado, a proposição 4.1 descrita abaixo assegura que tais pontos podem ser calculados de forma bastante eficiente.

Proposição 4.1 Se a mediana $h_{j}$ intercepta $m$ células do diagrama de Voronoi então o ponto $p_{h_{j}}$ pode ser calculado com no máximo $2 m-1$ cálculos de distância.

Prova. Sejam $v$ e $x$ os extremos de $h_{j}$, onde $v$ é um vértice da célula $d \sigma$ (na triangulação)

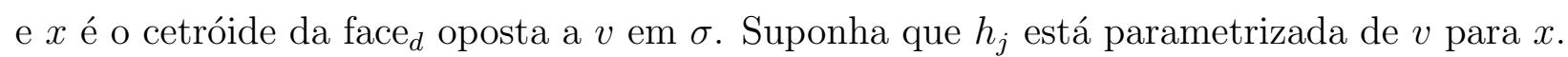
Por hipótese, a mediana $h_{j}$ intercepta $m$ células do diagrama de Voronoi. Desta forma, os pontos em $P^{h_{j}}$ podem ser divididos em $m$ subconjuntos $P_{i}^{h_{j}}$, cada um contendo os pontos de $P^{h_{j}}$ que estão em uma das $m$ células do diagrama de Voronoi. Como ilustrado na Figura 4.2 (para o caso bidimensional), a função $d_{\mathcal{D} \mathcal{T}}$ é quadrática por partes em $h_{j}$. Assim, em cada subconjunto $P_{i}^{h_{j}}$ o máximo de $d_{\mathcal{D} \mathcal{T}}$ ocorre ou no ponto mais a esquerda ou no ponto mais a direita de $P_{i}^{h_{j}}$ (considerando a parametrização), ou seja, apenas dois cálculos de distância são suficientes para encontrar o máximo de $d_{\mathcal{D} \mathcal{T}}$ em cada subconjunto. Um caso particular ocorre no subconjunto $P_{0}^{h_{j}}$, o qual contém os pontos da célula de Voronoi associada a $v$. Como $d_{\mathcal{D} \mathcal{T}}$ é igual a zero em $v$ (veja Figura $4.2(\mathrm{c})$ ), $d_{\mathcal{D} \mathcal{T}}$ precisa ser avaliada somente no ponto mais a direita de $P_{0}^{h_{j}}$.

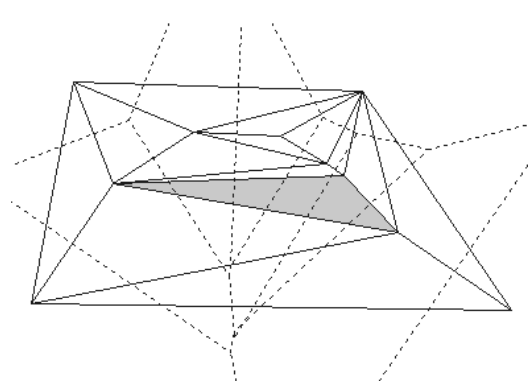

(a) Triangulação de Delaunay e Diagrama de Voronoi.

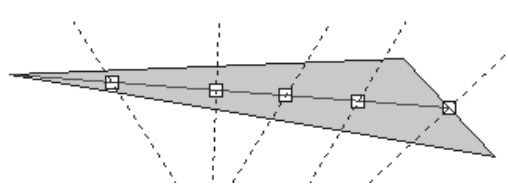

(b) Mediana de um triângulo interceptando as células de Voronoi.

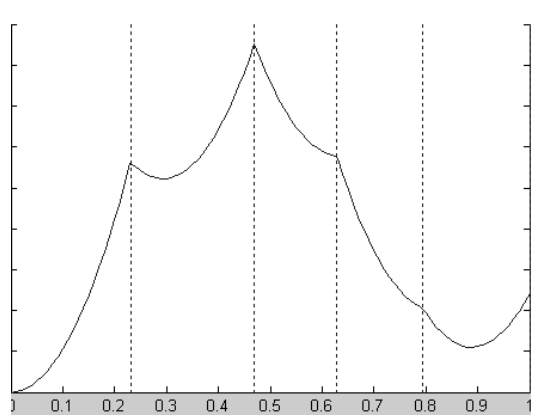

(c) $d_{\mathcal{D} \mathcal{T}}$ é quadrática por partes em $h_{j}$.

Figura 4.2: Comportamento da função $d_{\mathcal{D} \mathcal{T}}$. 
Embora a Proposição 4.1 forneça um mecanismo eficiente para calcular $p_{h_{j}}$, os cálculos de interseção entre $h_{j}$ e as células de Voronoi são inevitáveis, o que pode prejudicar a robustez do algoritmo. Uma alternativa que apresenta bons resultados práticos é calcular $p_{h_{j}}$ como o ponto em $P^{h_{j}}$ mais próximo ao circuncentro de $\sigma$. Nos testes que realizamos, aproximadamente $80 \%$ dos pontos que maximizam $d_{\mathcal{D} \mathcal{T}}$ são também os mais próximos do circuncentro, isto é, ambas as abordagens são equivalentes em aproximadamente $80 \%$ dos casos. A Figura 4.3 ilustra esse fato, sendo que a Figura 4.3(a) apresenta a imagem original. As Figuras 4.3(b) e 4.3(c), apresentam uma comparação entre duas malhas geradas com a estratégia de maior distância e utilizando o ponto próximo ao circuncentro, respectivamente $^{3}$. Em ambos os casos uma função de erro pontual de homogeneidade foi empregada. As Figuras $4.3\left(\right.$ d), (e) e (f), mostram resultados obtidos utilizando as medidas de erro $\mathscr{E}_{v}, \mathscr{E}_{g}$ e $\mathscr{E}_{i}$, respectivamente ${ }^{4}$.

\subsubsection{Detalhes e complexidade do processo de construção de malha}

A fim de simplificarmos a análise de complexidade do processo de construção de malha, assumiremos que a complexidade computacional envolvida no percorrimento das medianas para cada simplexo é dada por uma constante. Na verdade, podemos definir tal constante a partir do pior caso para o percorrimento de uma mediana, que ocorre em uma das diagonais do domínio $D$, ou seja, o custo de percorrer uma mediana é sempre inferior ao custo de percorrer a diagonal do domínio, que é uma constante para cada domínio $D$.

Além do custo de percorrer as medianas, a avaliação da função $E$ envolve o cálculo do ponto mais distante aos vértices já existentes na triangulação. Como mostrado na proposição 4.1, tal cálculo é proporcional ao número de células de Voronoi interceptadas pela mediana. Como pior caso, cada mediana intercepta todas as $n$ células de Voronoi existentes em cada passo ( $n$ o número de vértices).

Para completarmos a estimativa de complexidade do algoritmo, precisamos ter uma

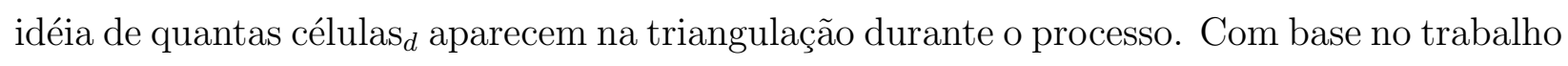
de Guibas et al. (1992), temos que o número esperado de triângulos gerados durante a construção da triangulação de Delaunay 2D pelo algoritmo incremental é $O(n)$. No caso 3D, supondo ainda o uso do algoritmo incremental para construção da triangulação, o número

\footnotetext{
${ }^{3}$ A função $\mathscr{E}_{v}$ foi utilizado, como exemplo, para realizar a comparação.

${ }^{4} \mathrm{O}$ cálculo do circuncentro foi utilizado como exemplo, para comparar os comportamentos.
} 


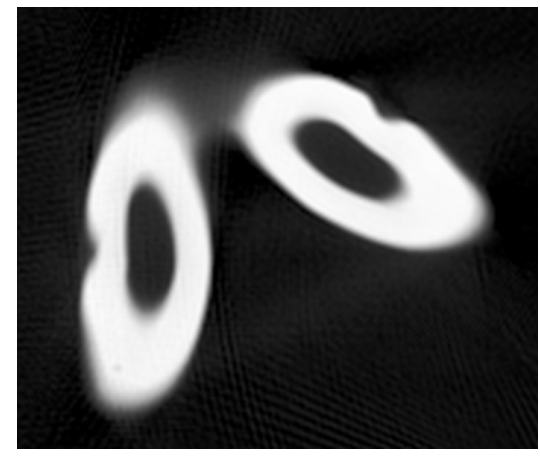

(a) Imagem original

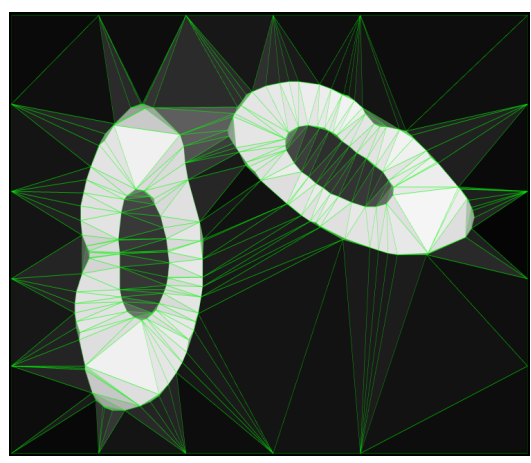

(d) Erro como medida de homogeneidade $\mathscr{E}_{v}, 225$ pontos.

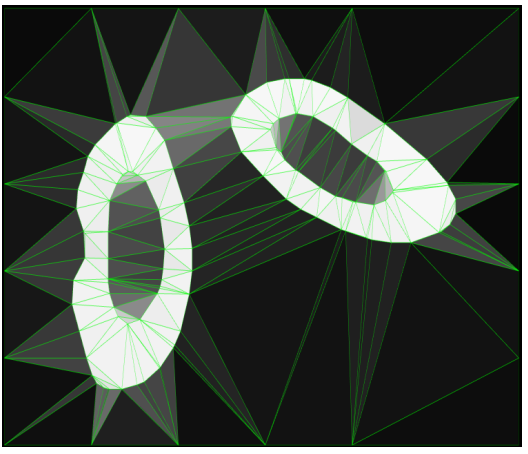

(b) Malha gerada utilizando o ponto mais distante, 146 pontos.

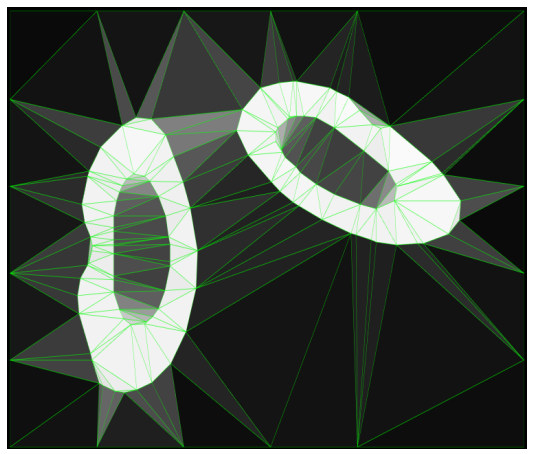

(c) Malha gerada utilizando o ponto mais próximo ao circuncentro, 137 pontos.

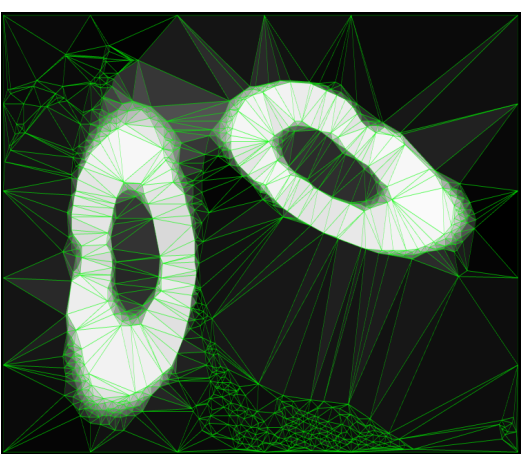

(e) Erro como medida de homogeneidade $\mathscr{E}_{g}, 1070$ pontos.

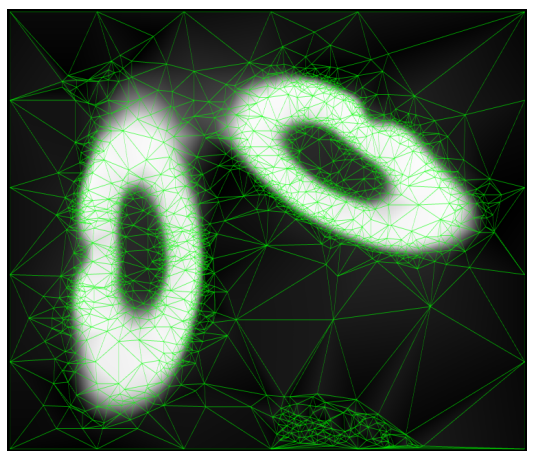

(f) Erro de interpolação linear $\mathscr{E}_{i}, 1936$ pontos.

Figura 4.3: Comparações entre os critérios de cálculos de distância, Figuras (b) e c(); e comparações entre cálculos de erro pontual, Figuras (d) (e) e (f).

de tetraedros esperados passa a ser de ordem quadrática, ou seja, $O\left(n^{2}\right)$. Como para cada célula $_{d}$ são executados, no pior caso, $O(n)$ cálculos de distância, a complexidade esperada do algoritmo é $O\left(n^{2}\right)$ para o caso bidimensional e $O\left(n^{3}\right)$ para o caso tridimensional.

Vale notar que a estimativa acima é bastante pessimista, uma vez que o número de células de Voronoi interceptadas por cada mediana é, em média, bastante inferior a $n$. 


\subsection{Particionamento de malha}

Tradicionalmente, métodos de particionamento de malha operam de forma puramente geométrica, não considerando atributos de imagem no processo (Yan et al., 2005; Zhang e Liu, 2005; Cohen-Steiner et al., 2004; Guillaume et al., 2004). Tais métodos buscam encontrar partições da malha que caracterizam estruturas geométricas de interesse no modelo, como regiões de alta curvatura ou quinas.

Poucos trabalhos tratam realmente do problema de segmentar malhas geradas a partir de imagens, sendo o trabalho de Bertin e colaboradores (Bertin et al., 1994) um dos poucos exemplos descritos na literatura. Diferente da abordagem proposta por Bertin, que utiliza uma subdivisão baseada em diagrama de Voronoi, o processo de particionamento proposto no Imesh visa rotular as células ${ }_{d}$ pertencentes a triangulação de modo a identificar os diferentes objetos contidos na imagem. Na verdade, o particionamento objetiva decompor a triangulação em um conjunto de sub-malhas, cada uma representando um objeto da imagem.

Um aspecto importante que diferencia o particionamento da triangulação de um processo convencional de segmentação é que, além dos atributos da imagem (como tonalidades de cor e texturas), propriedades geométricas obtidas da triangulação podem ser usadas na identificação das sub-malhas. De fato, o uso de propriedades geométricas tem sido muito pouco explorado em problemas de segmentação. Como veremos mais adiante, atributos geométricos podem ser decisivos em alguns casos.

Começaremos descrevendo, na Seção 4.2.1, as características da malha obtida pelo processo anterior de construção de malha. A seguir, duas estratégias distintas de particionamento são apresentadas. A Seção 4.2.2 descreve uma técnica que denominamos particionamento por operadores de agrupamento POpA e a Seção 4.2.3 apresenta uma extensão, para o contexto de malhas, da estratégia de segmentação de imagens baseada em campos aleatórios Markovianos, que denominaremos por PCAM. Consideraremos que as malhas apresentadas nesta Seção foram geradas utilizando as funções $E_{g}$ (Equação 4.5) ou $E_{v}$ (Equação 4.6).

\subsubsection{Características da malha a ser particionada}

Após o processo anterior de construção de malha (Seção 4.1), atributos da imagem contidos em cada célula $d$, como por exemplo cor, textura, histograma, etc., podem ser arran- 
jados em um vetor de características. Denomiramemos esse vetor associado a cada célula $d$ como "padrão de cor". O conceito de padrão de cor pode ser concretizado através de alguma medida estatística calculada com os dados contidos em cada célula ${ }_{d}$, como por exemplo, media, variância, etc.

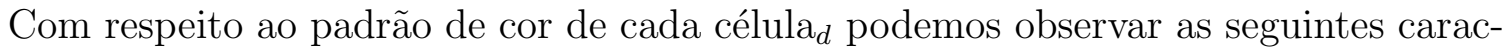
terísticas. Dependendo das características da imagem sendo tratada (ver Figura 4.4 (a)) e da função de erro pontual utilizada no processo de construção de malha (Seção 4.1), aparece na triangulação, em maior ou menor grau, um tipo particular de célula $a_{d}$ caracterizado por uma certa inconsistência no seu padrão de cor calculado a partir da imagem. Denominamos este grupo de célula $_{d}$ como "células $_{d}$ com ruído". Uma célula ${ }_{d}$ da malha se torna ruidosa quando esta está sobreposta entre regiões diferentes da imagem, sendo assim este tipo de célula $d$ pode apresentar um padrão de cor que não representa apropriadamente o seu posicionamento sobre a imagem. Mesmo quando funções de erro pontual de homogeneidade (Seção 4.1.1) são utilizadas, com o objetivo de reduzir a possibilidade de que células ${ }_{d}$ da triangulação atravessem objetos distintos da imagem, este fato não pode ser evitado totalmente, especialmente em

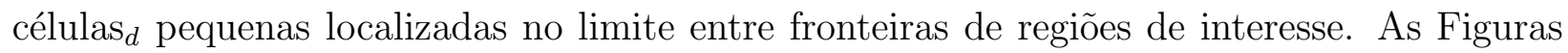
??(b),(c) (d) e (e) apresentam a aparição do fenômeno de células ${ }_{d}$ com ruído. Note que a existência de células ${ }_{d}$ com ruído não está relacionada à falta de aproximação do processo de

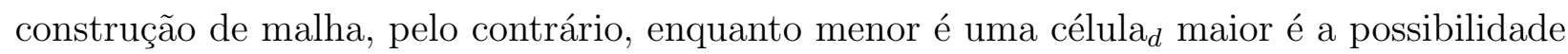
de que se torne ruidosa, já que poucos são os elementos (pixels ou voxels) necessários para desestabilizar o padrão de cor calculado no seu interior. Por exemplo, considere uma célula ${ }_{d}$ encapsulando unicamente quatro pixeis $_{d}$ no seu interior, bastariam somente dois deles para contaminar em 50\% o cálculo do padrão de cor. Vale ressaltar que, em alguns casos, a presença de células ${ }_{d}$ com ruído pode se tornar muito forte e caótica, especialmente para o

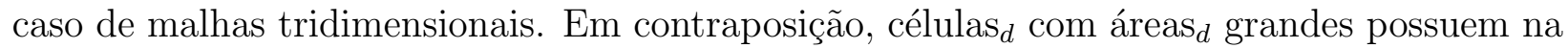
maioria dos casos, padrões de cor melhor definidos. Desta forma, é importante considerar, nos processos de particionamento, a idéia de confiabilidade do padrão de cor de uma célula em relação ao seu tamanho.

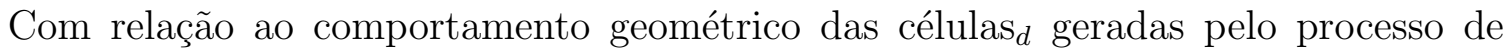
construção de malha, quando funções de erro pontual $E_{g}$ ou $E_{v}$ são empregadas, podemos observar as seguintes características. Em regiões homogêneas e grandes da imagem células $_{d}$ grandes tendem a aparecer. Em tal contexto, podemos dizer que células $_{d}$ dentro de 


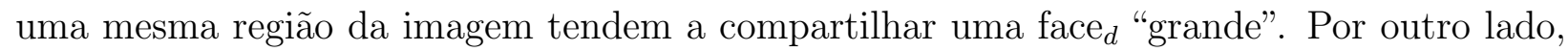
células $_{d}$ vizinhas em regiões distintas tendem a compartilhar uma face $_{d}$ "pequena". Este comportamento pode ser utilizado para criar um critério geométrico de similaridade entre células ${ }_{d}$ vizinhas da malha. Isto é, duas células $d$ poderiam ser consideradas similares quando compar-

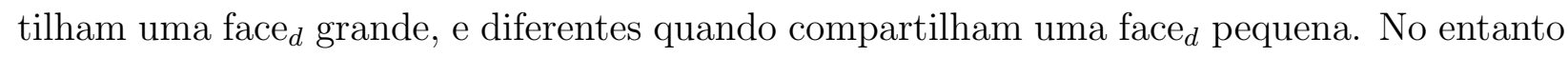

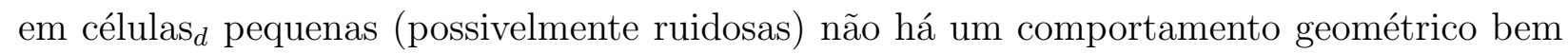
definido (ver Figura 4.4 (c)). Por outro lado, a presença na imagem de ruído, brilho, textura, etc., pode alterar totalmente o comportamento geométrico mencionado acima, como pode ser observado na Figura 4.4 (f).

Finalmente, a definição de célula ${ }_{d}$ ou região grande (ou pequena) é um fator que deve ser estabelecido dentro dos processos de particionamento de malha.

Os processos de particionamento apresentados a seguir aproveitam tanto padrões de cor quanto informações topológicas e geométricas contidos em cada célula ${ }_{d}$, considerando as características da malha discutidas acima.

\subsubsection{Particionamento por operadores de agrupamento (POpA)}

A técnica de particionamento baseada em operadores de agrupamento é uma idéia, criada nessa tese de doutorado, que está direcionada a resolver particularidades no contexto de particionamento de malhas criadas a partir de imagens. A técnica utiliza sub-processos, chamados de operadores, que dividem a malha aproveitando os padrões de cor definidos para cada célula $a_{d}$ e também informações topológicas e geométricas contidas na malha. Tais operadores podem interagir entre eles oferecendo diferentes alternativas de particionamento. A seguir, descrevemos esses sub-processos e suas possíveis combinações.

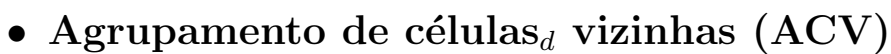

O objetivo deste processo é agrupar as células $d$ da malha $M$, em um conjunto de submalhas $M_{1}, \ldots, M_{s}$. Como mencionado anteriormente, os atributos da imagem contidos em cada célula ${ }_{d}$, ou conjunto de células ${ }_{d}$ (submalha), como por exemplo textura, cor e histograma, podem ser arranjados em um vetor de características denominado padrão de cor. Seja $\mathscr{S}_{I}$ uma função que compara tais vetores a fim de medir a similaridade entre sub-malhas. Denotamos por $C_{M}$ o vetor de características de uma sub-malha $M$, 


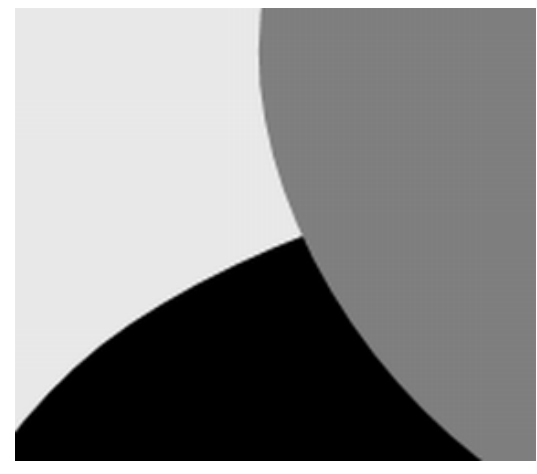

(a) Imagem de entrada com três regiões.

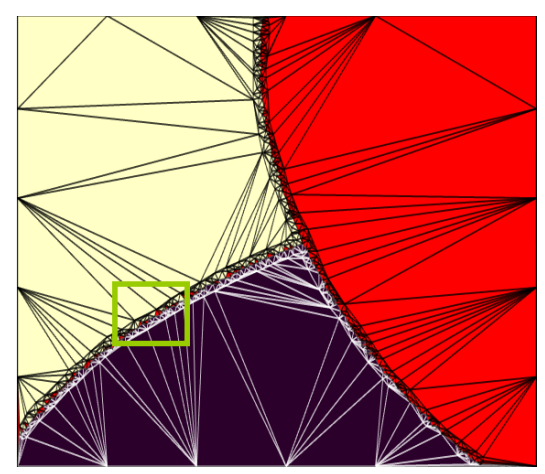

(d) Segmentação baseada somente em similaridade de cores.

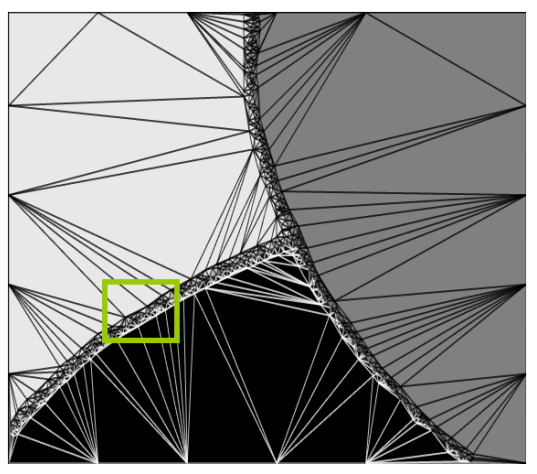

(b) Malha gerada utilizando a função de erro pontual $E_{g}(p)$.

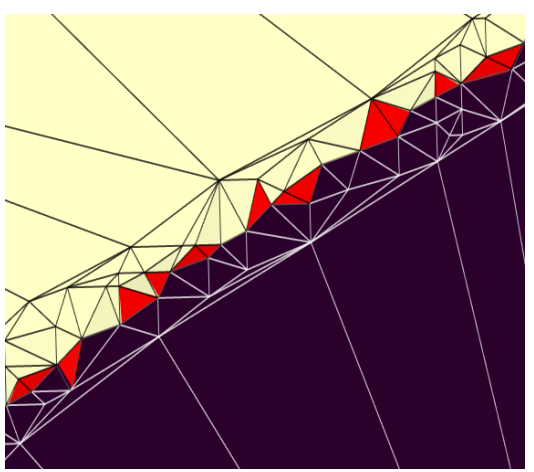

(e) Detalhe da área rectangular marcada na Figura (d)

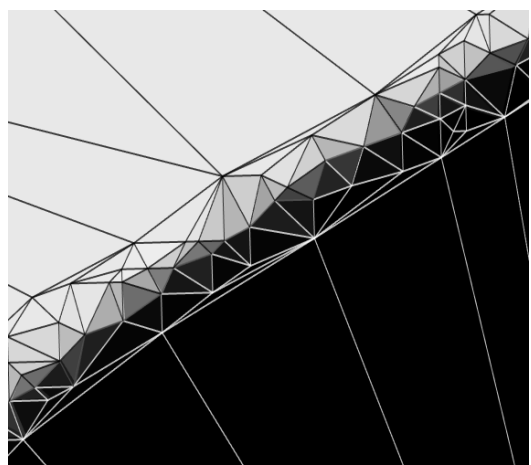

(c) Detalhe da área rectangular marcada na Figura (b)

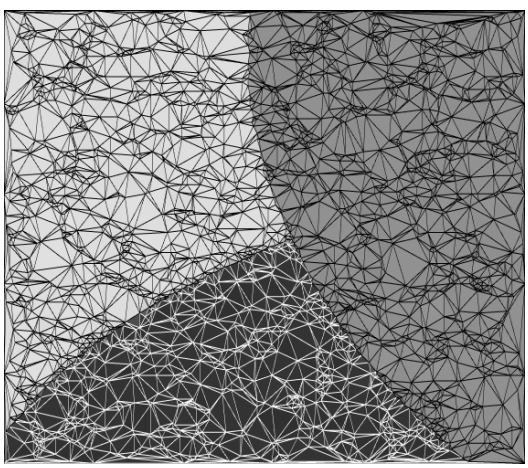

(f) Malha gerada com presença de ruído na imagem.

Figura 4.4: Células com ruído.

a função $\mathscr{S}_{I}$ pode ser definida como:

$$
\mathscr{S}_{I}\left(M_{i}, M_{j}\right)=1-\exp \left(-\frac{\left\|C_{M_{i}}-C_{M_{j}}\right\|^{2}}{\delta_{I}}\right)
$$

onde $\delta_{I}$ é um fator que controla a conduta de $\mathscr{S}_{I}$ em uma vizinhança de zero. Quanto maior o valor de $\delta_{I}$, mais lentamente $\mathscr{S}_{I}$ tende a zero. Note que $\mathscr{S}_{I}$ assume valores no intervalo $[0,1]$, onde valores próximos de zero significam sub-malhas similares. Este comportamento pode ser observado na Figura 4.5.

Fazendo uso da função de similaridade $\mathscr{S}_{I}$, e uma estratégia de segmentação por crescimento de regiões (Zucker, 2001; Hojjatoleslami e Kittler, 1998; Adams e Bischof, 1994), 


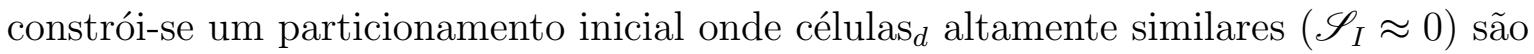
agrupadas em sub-malhas. Para isso, inicia-se uma sub-malha $M_{1}$ com uma célula ${ }_{d}$ $\sigma_{i}$ e mede-se a similaridade de $\sigma_{i}$ com suas células ${ }_{d}$ vizinhas $\sigma_{j}$ na triangulação. Se $\mathscr{S}_{I}\left(\sigma_{i}, \sigma_{j}\right) \approx 0$, então $\sigma_{j}$ é anexado à sub-malha $M_{1}$, e o padrão de cor $C_{M_{1}}$ é atual-

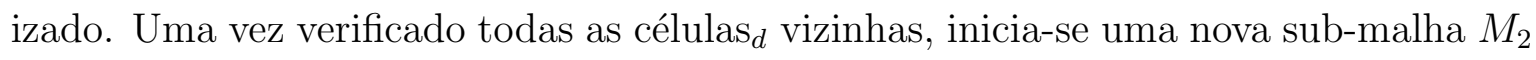
com uma célula $a_{d}$ ainda não agrupada, verificando sua similaridade com suas células ${ }_{d}$ vizinhas não pertencentes a $M_{1}$, anexando as altamente similares a $M_{2}$. O processo se repete até que todas as células ${ }_{d}$ tenham sido adicionadas a alguma sub-malha. Esta etapa de agrupamento é importante pois, em geral, produz um número de sub-malhas bastante inferior ao número de células ${ }_{d}$ da malha, permitindo simplificar operações em etapas posteriores.

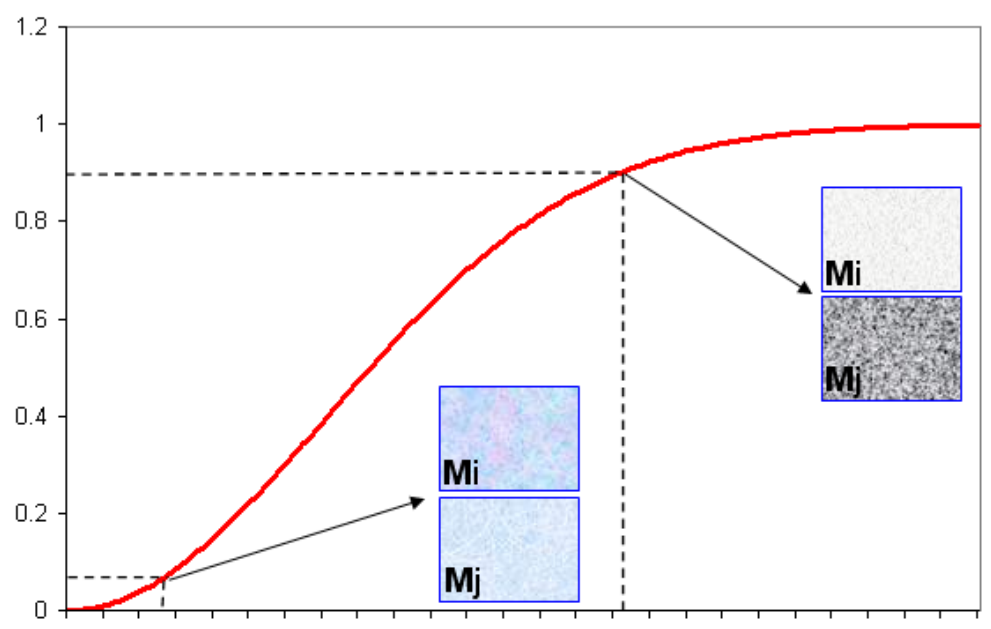

Figura 4.5: Comportamento da função $\mathscr{S}_{I}$ ao comparar pares de sub-malhas com padrões de cor com muita e pouca similaridade.

\section{- Agrupamento de sub-malhas vizinhas (ASmV)}

A partir do conjunto de sub-malhas gerado pelo processo anterior ACV, este passo do particionamento visa agrupar sub-malhas levando em consideração a vizinhança entre elas. Mais especificamente, nesta etapa, unicamente sub-malhas vizinhas serão fusionadas. Para estabelecer a ordem de uniões entre sub-malhas, definimos um critério $\gamma$ com o qual é possível ponderar a relevância de uma sub-malha durante as operações desta etapa de particionamento. O critério $\gamma$ de relevância para uma sub-malha $M_{i}$ pode ser definido a partir de dois fatores, área $d$ e número de células $d$, da seguinte 
maneira:

$$
\gamma\left(M_{i}\right)=\left(\frac{n c\left(M_{i}\right)}{n c(M)}\right)+\left(\frac{a\left(M_{i}\right)}{a(I)}\right)
$$

onde $n c$ e $a$ são funções que retornam o número de células ${ }_{d}$ e a área ${ }_{d}$ da sub-malha $M_{i}$, da malha $M$ e da imagem $I$, respectivamente.

A função $\gamma$ retorna um valor alto quando a sub-malha avaliada possui área grande e muitos elementos. No caso contrário, esta função atribuirá a uma sub-malha um valor baixo quando esta possui poucos elementos e área reduzida. A Figura 4.6, apresenta uma interpretação gráfica do resultado da função $\gamma$. A partir do critério $\gamma$ é possível criar uma fila de prioridade, onde os primeiros elementos são sub-malhas com área e número de células ${ }_{d}$ reduzidas, e os últimos elementos são sub-malhas com muitas

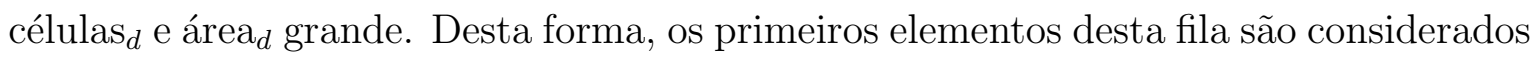
como os menos relevantes do conjunto. Após montada a fila de prioridade, a submalha menos relevante $M_{i}$ é unificada à sub-malha vizinha $M_{j}$ com a qual a função de similaridade $\mathscr{S}_{I}\left(M_{i}, M_{j}\right)$ seja mínima. Após a fusão da sub-malha menos relevante do conjunto, a fila de prioridade é atualizada e o processo é repetido até atingir um número desejado de sub-malhas.

A idéia por trás da ordem de fusões é que, como discutido na Seção 4.2.1, padrões de cor

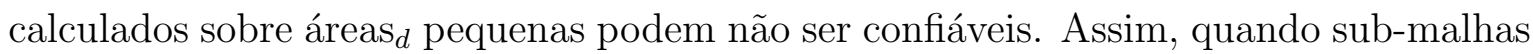
pouco relevantes são eliminadas a tendência é obter um conjunto menor de sub-malhas com padrões de cor mais robustos. Um resultado derivado do comportamento deste operador é que, objetos com o mesmo padrão de cor, mas separados na imagem, podem ser separados no particionamento, como mostra a Figura 4.7. Utilizando, por exemplo ASmV (7), para expressar uma redução do número de sub-malhas até 7 elementos, obtemos o resultado mostrado na Figura 4.7 (d).

\section{- Agrupamento de sub-malhas similares (ASmS)}

Este operador busca reduzir o número de elementos do conjunto de sub-malhas utilizando um critério de similaridade entre padrões de cor, e considerando idéias relacionadas ao algoritmo de agrupamento neighbor-joining (Saitou e Nei, 1987). Este processo de agrupamento pode ser utilizado para reduzir o número de elementos do 


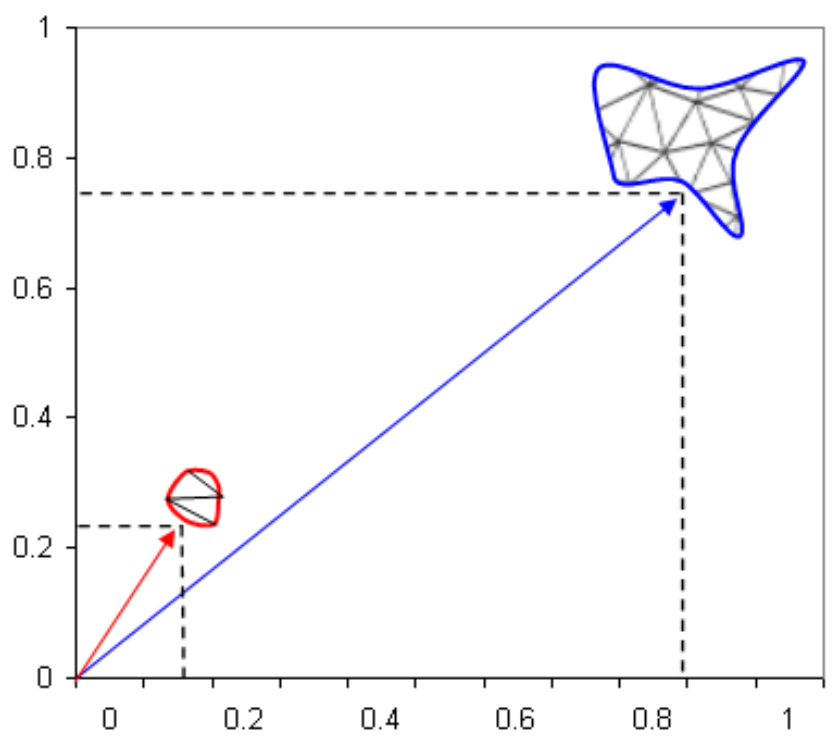

Figura 4.6: Comportamento da função $\gamma$ para estabelecer a relevância de duas sub-malhas, uma grande com várias células ${ }_{d}$ (distância maior) e uma de área pequena com poucas células ${ }_{d}$ (distância menor).

conjunto de sub-malhas gerado pelos processos ACV e ASmV. Para estabelecer a seqüência de uniões entre sub-malhas cria-se uma fila de prioridade, na qual é ordenada a similaridade de padrões de cor entre pares de sub-malhas. Mais claramente, para cada sub-malha $M_{i}$, calcula-se sua similaridade com as demais sub-malhas $M_{j}$, inserindo-as na fila de prioridade ordenada pelo valor da função $\mathscr{S}_{\mathscr{I}}\left(M_{i}, M_{j}\right)$, isto é, as duas submalhas mais similares aparecem no topo da fila. Uma vez montada a fila de prioridade, as duas sub-malhas no topo da fila são unificadas e um padrão de cor é criado para a nova sub-malha. Logo, o padrão de cor da nova sub-malha é confrontado com os padrões de cor das demais sub-malhas, e a fila de prioridades é atualizada. O processo continua até que se obtenha um número desejado de sub-malhas. Denotaremos, por exemplo, ASmS (4) para expressar uma redução até 4 sub-malhas por este operador. A Figura 4.8 apresenta o comportamento deste operador e como pode ser utilizado. As Figuras 4.8(a) e (b) apresentam a imagem de entrada e a malha obtida, respectivamente. A Figura 4.8 (c) apresenta um primeiro passo, utilizando o operador ACV. A Figura 4.8 (d) apresenta a aplicação do operador ASmS, e um mal comportamento por falta de uma etapa prévia de redução de ruído. A seguir, continuação se mostra na Figura 4.8 (e), a etapa de redução de ruído pelo operador ASmV é aplicada. Fi- 


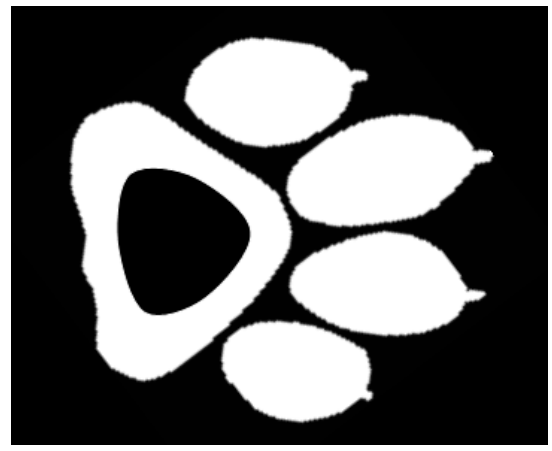

(a) Imagem original

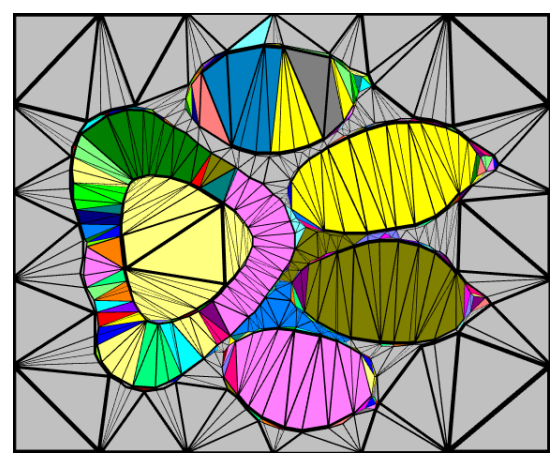

(c) $\mathrm{ACV}$

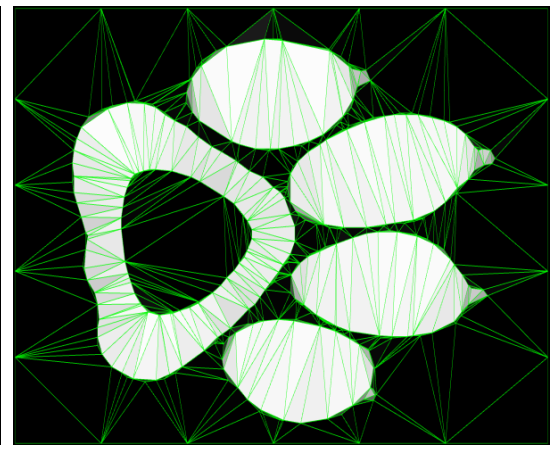

(b) Malha gerada

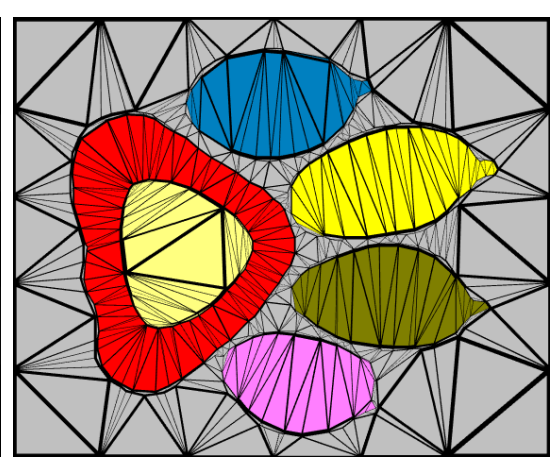

(d) $\mathrm{ACV}+\mathrm{ASmV}(7)$

Figura 4.7: Particionamento utilizando o operador ASmV.

nalmente, a Figura 4.8 (f) mostra a aplicação do operador ASmS após a etapa de tratamento de ruído.

\section{- Agrupamento de células $d$ por critérios geométricos (ACG)}

O objetivo deste oeprador de agrupamento é tirar vantagem da conduta geométrica da malha produzida pela etapa de construção de malha. Quando a malha construída possui um comportamento geométrico bem definido, é possível adotar um critério ge-

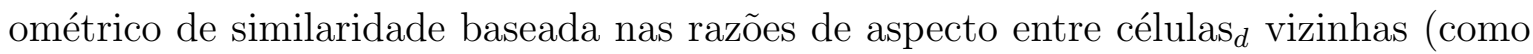
discutido na Seção 4.2.1). Por exemplo, a presença de um grupo de células grandes na malha indica a existência de uma região grande e homogênea na imagem. Em tal contexto, células ${ }_{d}$ dentro de uma mesma região da imagem tendem a compartilhar

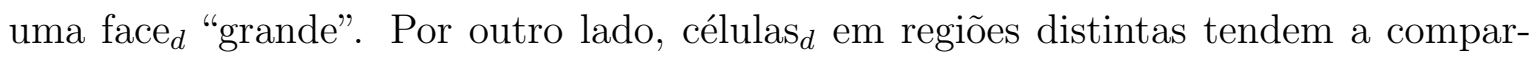




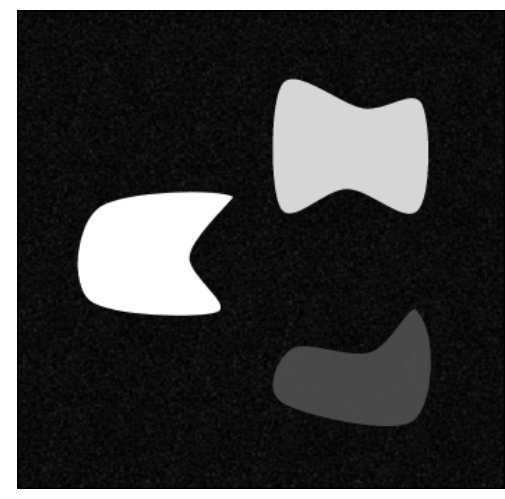

(a) Imagem original

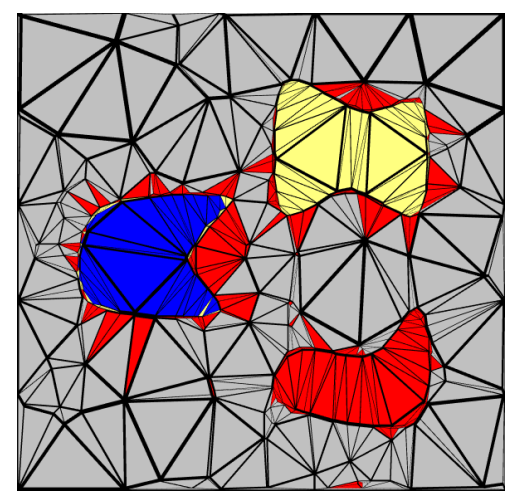

(d) $\mathrm{ACV}+\mathrm{ASmS}(4)$

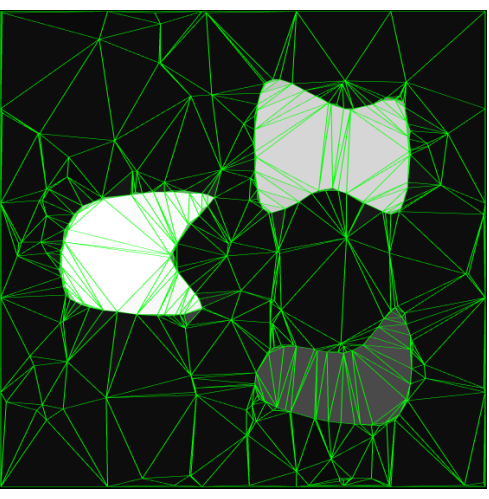

(b) Malha gerada

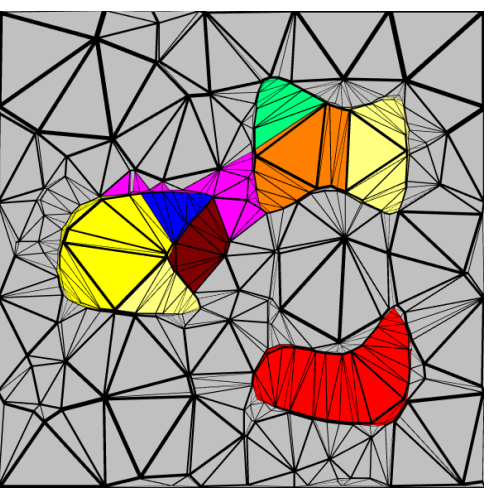

(e) $\mathrm{ACV}+\mathrm{ASmV}(10)$

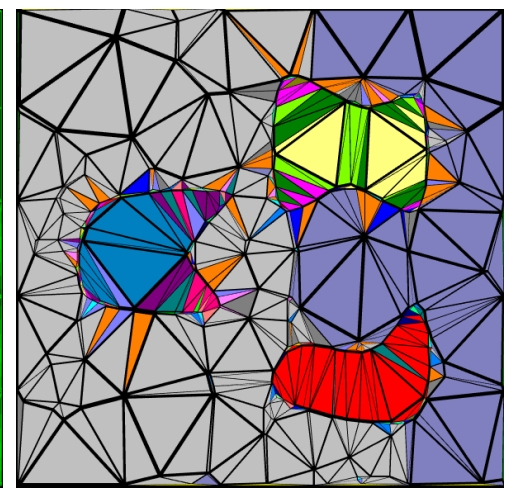

(c) $\mathrm{ACV}$

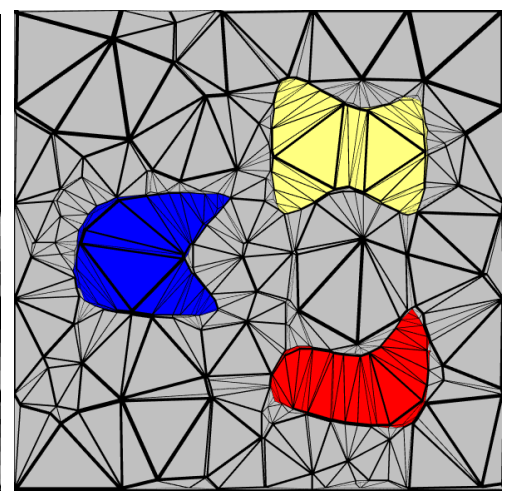

(f) $\mathrm{ACV}+\mathrm{ASmV}$

(10)+ASmS (4)

Figura 4.8: Particionamento utilizando o operador ASmS.

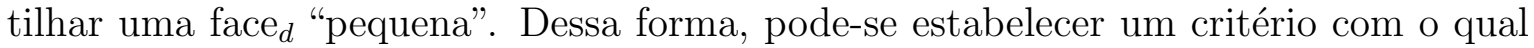

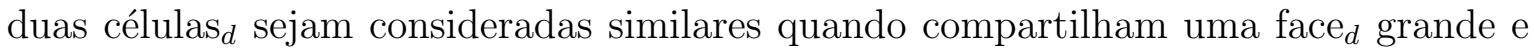
diferentes quando compartilham um face pequena.

O conceito de "grande" e "pequena" é convertido, em termos numéricos, por meio do comprimento de arestas. No caso bidimensional, utiliza-se o comprimento da aresta compartilhada por dois triângulos. No caso tridimensional, o comprimento da maior aresta do triângulo compartilhado por dois tetraedros é usada como medida de tamanho. Com base na discussão acima, definimos $\mathscr{S}_{G}$ como segue:

Sejam $e_{r}$ e $e_{s}$ a maior aresta das células $\sigma_{r}$ e $\sigma_{s}$, respectivamente. Se $\sigma_{r}$ e $\sigma_{s}$ com- 
partilham uma face $f$, denote por $e_{f}$ a maior aresta de $f$ (no caso 2D, $f$ é a própria aresta $\left.e_{f}\right)$ e considere:

$$
\phi(f)=\left\{\begin{array}{cl}
\frac{1}{2}\left(\frac{\left|e_{f}\right|}{\left|e_{r}\right|}+\frac{\left|e_{f}\right|}{\left|e_{s}\right|}\right) & \text { se }\left(\left|e_{r}\right|>e_{\min } \text { ou }\left|e_{s}\right|>e_{\text {min }}\right) \\
-1 & \text { caso contrário }
\end{array}\right.
$$

uma medida de razão de aspecto entre $\sigma_{r}$ e $\sigma_{s}$, onde o comprimento de uma aresta $e$ é denotado como $|e|$, e $e_{\min }$ expressa um limiar, de comprimento de aresta mínima, que é considerado na operação. Para cada par de sub-malhas $M_{i}$ e $M_{j}$, a similaridade geométrica pode ser calculada da seguinte maneira:

$$
\mathscr{S}_{G}\left(M_{i}, M_{j}\right)=\left\{\begin{array}{cl}
1 & \text { se } M_{i} \cap M_{j}=\emptyset \\
-1 & \text { se } \forall f \in M_{i} \cap M_{j}, \phi(f)=-1 \\
\frac{1}{n_{f}} \sum_{\substack{f \in M_{i} \cap M_{j} \\
\phi(f) \neq-1}} \exp \left(-\frac{\phi(f)^{2}}{\delta_{G}}\right) & \text { se } M_{i} \cap M_{j} \neq \emptyset
\end{array}\right.
$$

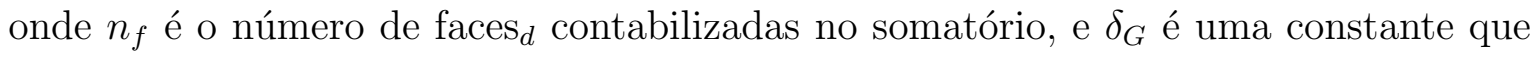
controla a conduta de $\mathscr{S}_{G}$ em na vizinhança de zero. Note que, novamente, duas sub-malhas são consideradas similares (em termos geométricos) se $\mathscr{S}_{G}$ assume valores próximos de zero. A figura Figura 4.9 ilustra a função $\mathscr{S}_{G}$ no caso bidimensional, considerando $M_{i}$ e $M_{j}$ constituídos de um triângulo cada. Observe que a função $\mathscr{S}_{G}$ assume valores próximos de um quando dois triângulos compartilham uma aresta pequena.

A partir da função de similaridade $\mathscr{S}_{G}$, pode-se utilizar uma estratégia similar à aplicada no operador ACV. Isto é, pode-se agrupar células ${ }_{d}$ e sub-malhas utilizando uma abordagem por crescimento de região, onde $\mathscr{S}_{G} \approx 0^{5}$.

Considere agora, uma imagem na qual existam dois objetos com padrões de cor iguais (ou muito similares) e que estejam unidos apenas por uma pequena região de interseção, como o exemplo da Figura 4.10 (a). Utilizando operações de agrupamento baseadas em similaridade de padrões de cor (ACV,ASmS), estes dois objetos não poderiam ser separados. Da mesma forma, já que tais objetos possuem uma região em comum, também não poderiam ser separados utilizando um operador baseado em vizinhança

\footnotetext{
${ }^{5}$ Se para duas sub-malhas $M_{i}$ e $M_{j}, \mathscr{S}_{G}\left(M_{i}, M_{j}\right)=-1$, nenhuma união é realizada
} 


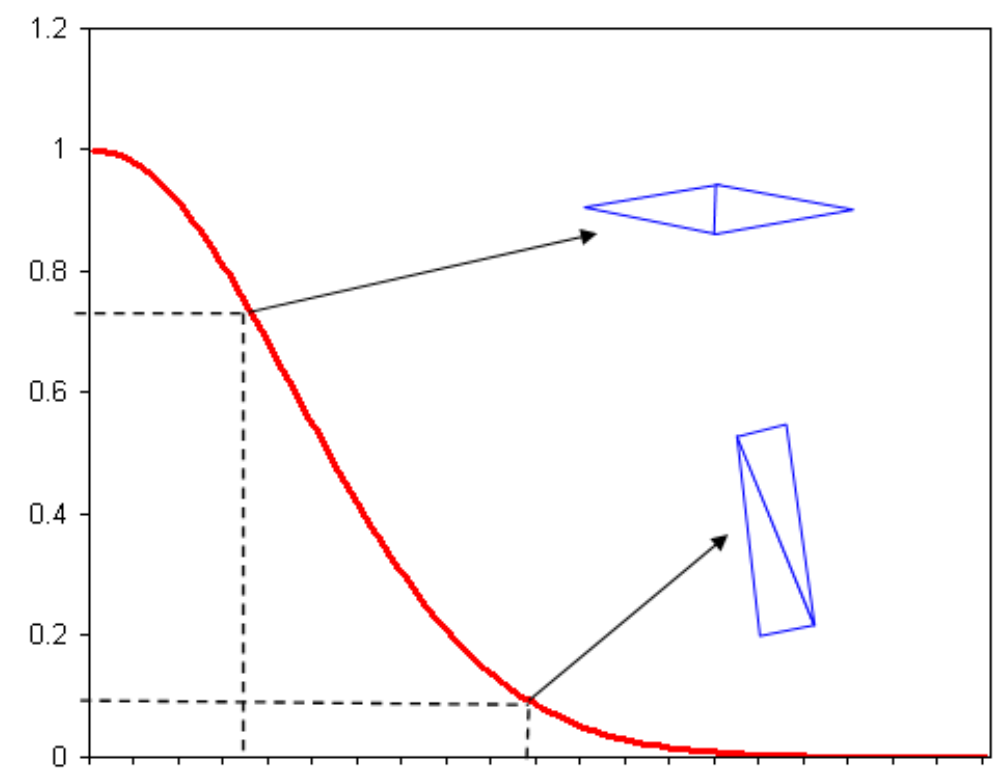

Figura 4.9: Conduta da função $\mathscr{S}_{G}$ ao comparar dois triângulos.

de sub-malhas (ASmV). No entanto pode-se tirar vantagem do comportamento geométrico da malha (ver Figura 4.10 (b)), da seguinte maneira: Primeiro, aplica-se o operador ACG, formando três regiões grandes onde a geometria é bem comportada, como mostra a Figura 4.10 (c), onde ACG (17) representa o processo com $e_{\text {min }}=17$. Os espaços escuros da imagem representam células ${ }_{d}$ ainda não rotuladas. Segundo, as células $_{d}$ ainda não consideradas são rotuladas utilizando o operador ACV, como mostra a Figura $4.10(d)$.

Finalmente, como o critério utilizado no operador ASmV garante a permanência de sub-malhas mais relevantes, sub-malhas menores podem ser eliminadas e podemos obter quatro sub-malhas (os três objetos importantes e o fundo). O resultado final deste particionamento pode ser apreciado na Figura 4.10 (e).

\section{- Agrupamento de células $_{d}$ com barreira de restrição (BR)}

Existem imagens nas quais fronteiras entre objetos podem estar levemente definidas. Este fato pode comprometer o bom desempenho das operações de particionamento. Considere, por exemplo, a imagem da Figura 4.11 (a) e suponha que o objetivo do particionamento seja separar o objeto arredondado da imagem, do objeto da parte inferior da imagem (abjeto alongado). A Figura 4.11 (b) apresenta o resultado do 


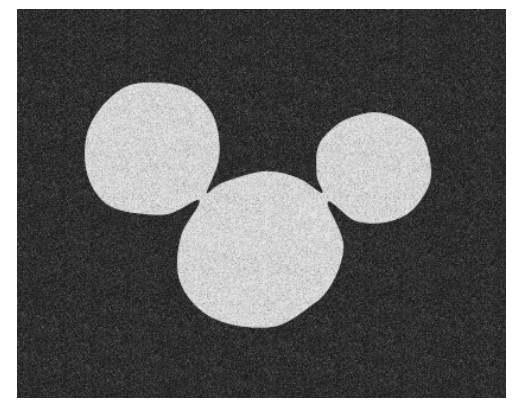

(a) Imagem original

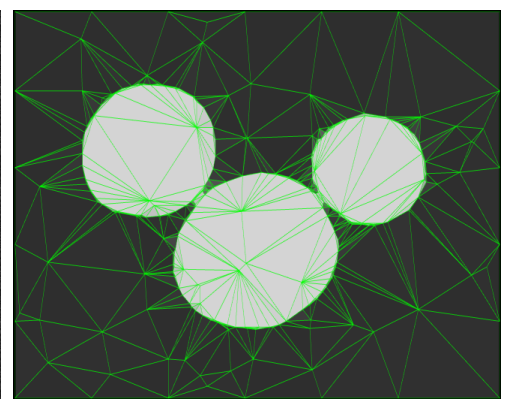

(b) Malha gerada

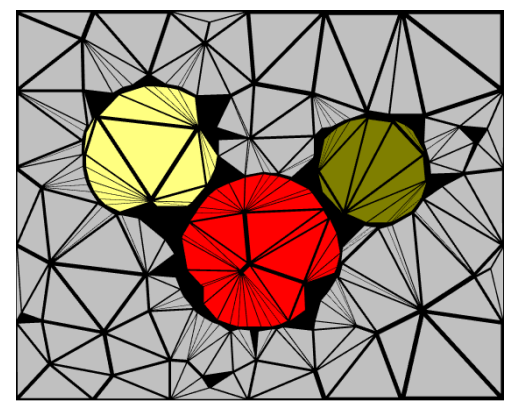

(c) ACG

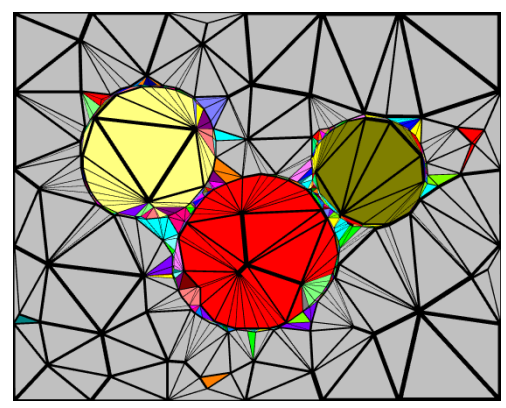

(d) ACG (17)+ACV

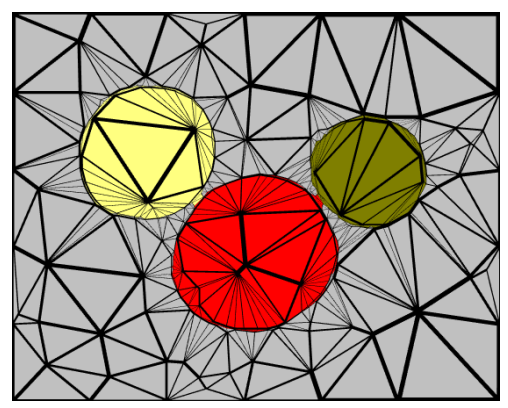

(e) $\mathrm{ACG} \quad(17)+\mathrm{ACV}$ $+\mathrm{ASmV}(4)$

Figura 4.10: Particionamento utilizando o operador ACG.

processo de construção de malha. Como pode-se apreciar na malha gerada, os padrões de cor de células ${ }_{d}$ próximas à fronteira, entre os dois objetos, são muito parecidos, podendo causar confusão para os critérios baseados em similaridade de padrões de cor $(\mathrm{ACV}, \mathrm{ASmS})$. Esta similaridade de padrões de cor, somado à proximidade de células ${ }_{d}$ dos dois objetos em questão, pode também causar confusão para as operações de fusão entre sub-malhas vizinhas $(\mathrm{ASmV})$. Finalmente, as células $d$ próximas às fronteiras e entre os dois objetos não possuem um comportamento geométrico apropriado para determinar a separação entre regiões. A Figura 4.11 (c) apresenta um exemplo do resultado de um particionamento realizado com alguma ${ }^{6}$ combinação dos operadores anteriormente mencionados.

Por outro lado, especialmente quando a operação $H_{v}$ (Equação 4.4) é utilizada dentro

\footnotetext{
${ }^{6}$ Foi utilizado como exemplo:ACV $+\mathrm{ASmV}(13)+\mathrm{ASmS}(3)$
} 
do processo de construção de malha, como neste caso, abre-se uma nova possibilidade para evitar o comportamento não desejado descrito acima. Na Figura 4.11 (d) podemos observar o conjunto de pontos considerados de fronteira pela operação $H_{v}$, para a imagem da Figura 4.11 (a). Tais fronteiras foram utilizadas para posicionar pontos durante o processo de construção de malha. A partir deste conjunto de pontos pode-se notar com mais clareza a separação entre os objetos da imagem. Mais ainda, este conjunto de pontos representa uma barreira que as operações de agrupamento não deveriam ter ultrapassado durante o particionamento, gerando o resultado não desejado apresentado na Figura 4.11 (c). Desta forma, se nós conseguirmos representar esta mesma barreira dentro da malha, esta poderia ser utilizada como uma barreira de restrição, que certas operações de agrupamento poderiam considerar. Assim, poderiase evitar que durante a formação de sub-malhas, fronteiras importantes de objetos presentes na malha sejam atravessadas. Alternativas de como transferir a idéia da barreira de restrição ao contexto da malha são explicadas a seguir.

Considere o mesmo conjunto de iso-valores, $\Psi=\left\{v_{0}, v_{1}, \ldots, v_{n}\right\}$, que foram utilizados pela função $H_{v}$ para definir pontos de fronteira na imagem $I$, durante o processo de construção de malha. Sejam $\sigma_{i}$ e $\sigma_{j}$ duas células $d$ vizinhas na malha $M$, que compartilham uma face $f_{i j}$. No contexto da malha, denominaremos por $H_{M v}$ à operação binária para determinar se uma face $f_{i j}$ é (valor 1 ) ou não é (valor 0) uma iso-face . $_{\text {. }}$ Desta forma, considere a barreira de restrição representada pelo conjunto de iso-faces ${ }_{d}$ $B R=\left\{f_{i j} \in M \mid H_{M v}\left(f_{i j}\right)=1\right\}$. A função $H_{M v}$ pode ser criada a partir de dois critérios, os quais são descritos a seguir.

\section{- Critério da poli-linha}

Sejam $\kappa_{\sigma_{i}}, \kappa_{\sigma_{j}}, \kappa_{f_{i j}}$, os centróides das células $\sigma_{d} \sigma_{i}, \sigma_{j}$, e da face $f_{i j}$, respectivamente. Considere a poli-linha $L$ formada pelos pontos $\kappa_{\sigma_{i}}, \kappa_{f_{i j}}$ e $\kappa_{\sigma_{j}}$; e seja $P=\left\{p_{1}, p_{2}, \ldots, p_{m}\right\}$ o conjunto de pontos da imagem $I$, que formam $L$. O critério $H_{M v}^{p l}$, baseado na poli-linha $L$, determina se a face $f_{i j}$ é ou não uma iso-face ${ }_{d}$, da seguinte maneira:

$$
H_{M v}^{p l}\left(f_{i j}\right)= \begin{cases}1 & \text { se } \exists p \in P \mid I(p) \in \Psi \\ 1 & \text { se } \left.\exists v_{i} \in \Psi ; p_{k}, p_{k-1} \in P \mid v_{i} \in\right] I\left(p_{k}\right), I\left(p_{k-1}\right)[ \\ 0 & \text { caso contrário }\end{cases}
$$




\section{- Critério de padrões de cor}

Este critério pode ser utilizado considerando que o cálculo aplicado, para representar os padrões de cor das células ${ }_{d}$ da malha $M$, é a média de cores contidas

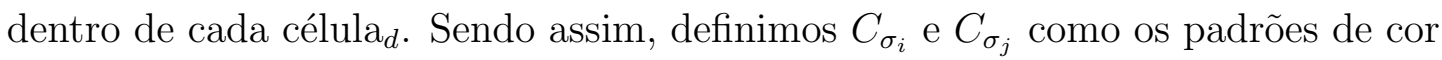
das células $_{d} \sigma_{i}$ e $\sigma_{j}$, respectivamente. Então, a função definida a partir de padrões de cor $H_{M v}^{p c}$, decide se uma face $f_{i j}$ é uma iso-face ${ }_{d}$, como segui:

$$
H_{M v}^{p c}\left(f_{i j}\right)= \begin{cases}1 & \text { se } C_{\sigma_{i}} \in \Psi \text { ou } C_{\sigma_{j}} \in \Psi \\ 1 & \text { se } \left.\exists v_{i} \in \Psi \mid v_{i} \in\right] C_{\sigma_{i}}, C_{\sigma_{j}}[ \\ 0 & \text { caso contrário }\end{cases}
$$

Dependendo das características da imagem e da malha gerada, pode ser mais apropriado utilizar $H_{M v}^{p c}$ ou $H_{M v}^{p l}$. Em termos de precisão na definição da fronteira, o critério $H_{M v}^{p c}$, é mais apropriado quando a imagem possui textura ou ruído, pois este fato pode desestabilizar o cálculo pelo critério $H_{M v}^{p l}$. No entanto, $H_{M v}^{p c}$ se torna instável dependendo da quantidade de ruído presente nas células $d$ da malha. Já o cálculo $H_{M v}^{p l}$ é mais estável ao tratar células ${ }_{d}$ com ruído, pois este considera fronteiras na imagem e não o padrão de cor das células ${ }_{d}$. Com respeito à velocidade, o cálculo $H_{M v}^{p c}$, baseado unicamente na comparação de dois valores numéricos, é um cálculo mais leve do que $H_{M v}^{p l}$, que considera um conjunto de valores no percurso de uma poli-linha. Normalmente, a diferença entre $H_{M v}^{p l}$ e $H_{M v}^{p c}$, para o caso bidimensional pode ser mínima, em tempo e precisão. No caso tridimensional a diferença de tempo é maior, e a precisão pode ser afetada mais facilmente, pois a presença de ruído nas células ${ }_{d}$ é mais intensa. A Figura 4.11 (e) apresenta a barreira de restrição obtida para a malha da Figura 4.11 (b), utilizando uma função $H_{M v}{ }^{7}$, o resultado da aplicação da função $H_{M v}^{p l}$ é muito parecido.

Depois de ter representado a barreira de restrição na malha, por um conjunto de iso-faces $_{d}$, esta barreira, como dito anteriormente, pode servir como uma referência para evitar que sub-malhas atravessem fronteiras importantes de objetos da malha, durante as operações do particionamento. Particularmente, é muito útil restringir as

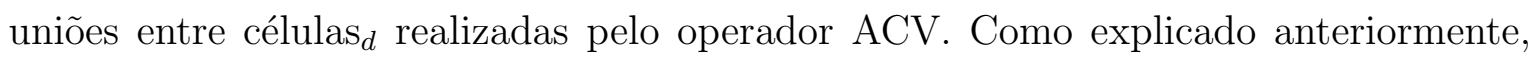
o operador ACV realiza uniões entre células $d$ utilizando a função de similaridade $\mathscr{S}_{I}$ (Equação 4.7). Lembre que a função $\mathscr{S}_{I}$ retorna valores próximos a zero quando os

\footnotetext{
${ }^{7}$ Neste caso foi utilizado a função $H_{M v}^{p c}$
} 
elementos comparados possuem padrões de cor considerados similares. Com base em $\mathscr{S}_{I}$, é possível criar uma nova operação, que denominaremos $\mathscr{S}_{B R}$, que considere a barreira de restrição para determinar se duas células ${ }_{d}$ devem ou não ser unificadas, da seguinte maneira:

$$
\mathscr{S}_{B R}\left(\sigma_{i}, \sigma_{j}\right)= \begin{cases}1 & \text { se } H_{M v}\left(f_{i j}\right)=1 \\ \mathscr{S}_{I}\left(\sigma_{i}, \sigma_{j}\right) & \text { caso contrário }\end{cases}
$$

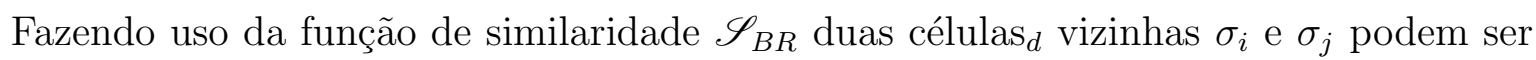
consideradas similares unicamente quando não existe nenhum elemento da barreira de restrição $B R$ passando entre elas. Mais ainda, se atribuirmos um valor alto a $\delta_{I}$, estaremos permitindo que células ${ }_{d}$ distantes, em termos de padrões de cor, sejam consideradas similares. Por outro lado, com $\delta_{I}$ com um valor alto, as uniões entres células $_{d}$ passam a ser controladas principalmente pela barreira $B R$. Desta forma, denominaremos $\mathrm{ACV}_{B R}$ ao operador $\mathrm{ACV}$ que utiliza a função de similaridade $\mathscr{S}_{B R}$ dentro dos seus cálculos.

Repare que, teoricamente, a idéia da barreira de restrição pode ser aplicada também para modificar as operações de agrupamento ACG e ASmV, surgindo as operações $\mathrm{ACG}_{B R}$ e $\mathrm{ASmV}{ }_{B R}$, respectivamente. No entanto, não foram registrados casos nos quais a aplicação de tais operações seja realmente necessária.

A Figura 4.11 (f), apresenta o resultado da aplicação do operador $\mathrm{ACV}_{B R}$, como descrito acima. Observe que a utilização deste operador, como primeiro passo do particionamento, já separa os dois objetos da malha, o arredondado (acima) e o alongado (abaixo). Após deste primeiro passo, o particionamento pode continuar com os operadores de agrupamento descritos anteriormente. A Figura 4.11 (g) apresenta a aplicação do operador ASmV ao resultado mostrado na Figura 4.11 (f). Finalmente, a aplicação do operador ASmS ao resultado da Figura 4.11 (g) é mostrado na Figura 4.11 (h).

\subsubsection{Particionamento por campos aleatórios Markovianos (PCAM)}

Na Seção 3.3 foram descritas as idéias gerais do método EM/MPM para segmentação de imagens. Apresentaremos nesta Seção uma adaptação desse método para o contexto de triangulações. Embora as triangulações geradas a partir de imagens possuam as propriedades 


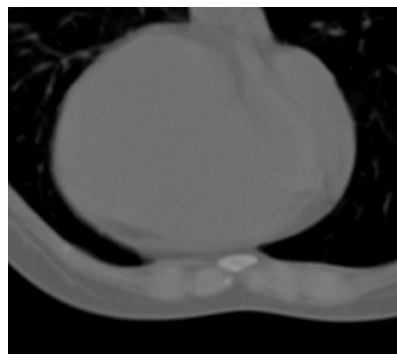

(a) Imagem original

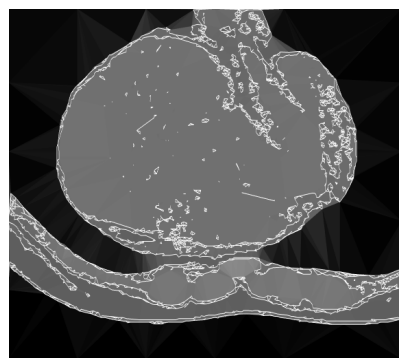

(e) Iso-contornos na malha

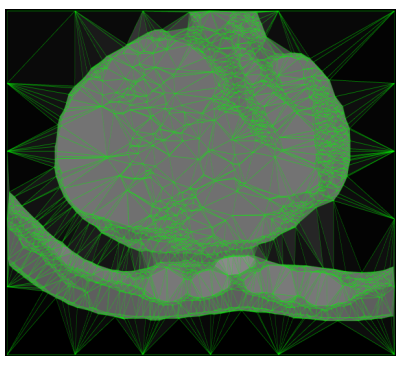

(b) Malha gerada

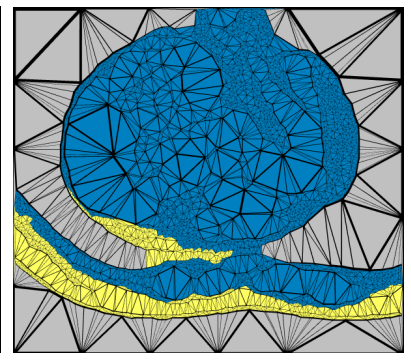

(c) Operadores anteriores

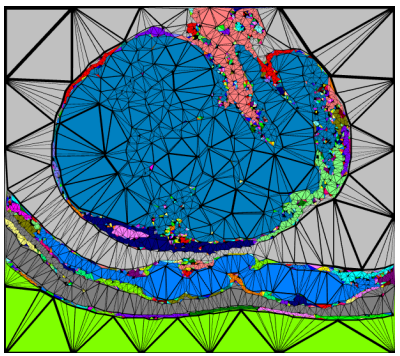

(f) $\mathrm{ACV}_{B R}$

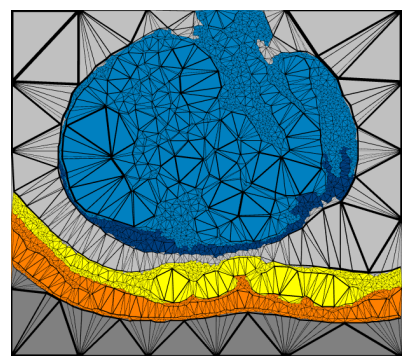

(g) $\mathrm{ACV}+\mathrm{ASmV}(6)$

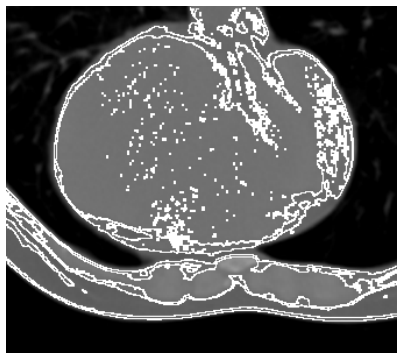

(d) Iso-contornos na imagem

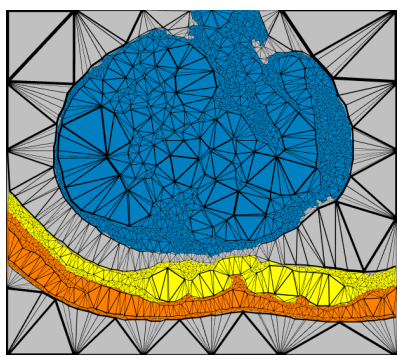

(h) $\mathrm{ACV}+\mathrm{ASmV}$ (6) $+\mathrm{ASmS}(4)$

Figura 4.11: Particionamento utilizando barreira de restrição.

necessárias para o emprego de uma segmentação via EM/MPM, o uso desse esquema de segmentação em triangulações não tem sido descrito na literatura, sendo este (até onde temos conhecimento) o primeiro trabalho a empregar tal abordagem.

\section{Adaptação do EM/MPM para triangulações}

No caso de triangulações pode-se empregar a estratégia EM/MPM de forma direta, apenas redefinindo quem são os elementos adjacentes (vizinhos). Porém, como visto anteriormente, na Seção 4.2.2, informações geométricas podem ser de grande importância no contexto de triangulações. Assim, propomos uma modificação (Cuadros-Vargas et al., 2006a) no método EM/MPM de modo a considerar, além dos atributos da imagem, informações geométricas oriundas da triangulação.

No procedimento $M P M$, informações geométricas dos elementos da malha são incorporadas na função penalidade de modo a penalizar atribuição de rótulos diferentes para 
células $_{d}$ que compartilham uma aresta "grande".

No caso bidimensional, utiliza-se o comprimento da aresta compartilhada por dois triângulos. No caso tridimensional, o comprimento da maior aresta do triângulo compartilhado por dois tetraedros é usada como medida de tamanho. Com base nessa idéias podemos definir a razão de aspecto entre duas células ${ }_{d}, \sigma_{i}$ e $\sigma_{j}$, como segue:

Sejam $e_{i}$ e $e_{j}$ as maiores arestas das células $\sigma_{i}$ e $\sigma_{j}$. Se $\sigma_{i}$ e $\sigma_{j}$ compartilham uma face $_{d} f$, denote por $e_{f}$ a maior aresta de $f$ (no caso $2 \mathrm{D}, f$ é a própria aresta $e_{f}$ ) e defina a função razão de aspecto $\hat{\phi}(f)$, de forma similar a Equação 4.9, como segue $^{8}$ :

$$
\hat{\phi}(f)=\frac{1}{2}\left(\frac{\left|e_{f}\right|}{\left|e_{i}\right|}+\frac{\left|e_{f}\right|}{\left|e_{j}\right|}\right)
$$

onde $|e|$ é o comprimento da aresta $e$.

Dessa forma, para o caso de triangulações, considerando um par de células ${ }_{d}$ compar- $^{-}$ tilhando uma face ${ }_{d}$, a função penalidade (3.7) pode ser re-escrita como:

$$
\hat{t}\left(x_{\sigma_{i}}, x_{\sigma_{j}}\right)= \begin{cases}0 & \text { se } x_{\sigma_{i}}=x_{\sigma_{j}} \\ \hat{\phi}(f) & \text { se } x_{\sigma_{i}} \neq x_{\sigma_{j}}\end{cases}
$$

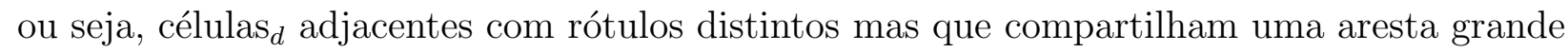
são penalizados.

Informações geométricas são também incorporadas no procedimento $E M$. No caso de imagens, a cor (nível de cinza) de cada elemento tem a mesma importância (peso) no

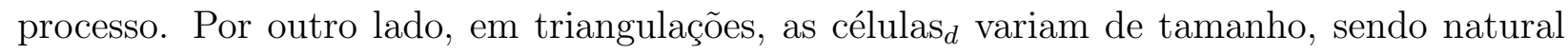
atribuir pesos diferentes para células ${ }_{d}$ com tamanhos diferentes. Assim, para o caso de triangulações, a estimativa dos parâmetros $\theta$ (veja Equação 3.12) torna-se:

$$
\begin{gathered}
\mu_{r}(n+1)=\frac{1}{N_{r_{V o l}}(n+1)} \sum_{i=1}^{N} y_{i} p_{X_{\sigma_{i}} \mid \mathbf{Y}}(r \mid \mathbf{y}, \theta(n)) \operatorname{Vol}\left(\sigma_{i}\right) \\
\delta_{r}^{2}(n+1)=\frac{1}{N_{r}(n+1)} \sum_{i=1}^{N}\left(y_{\sigma_{i}}-\mu_{r}(n+1)\right)^{2} p_{X_{\sigma_{i}} \mid \mathbf{Y}}(r \mid \mathbf{y}, \theta(n))
\end{gathered}
$$

\footnotetext{
${ }^{8}$ No caso da função $\phi$ (Equação 4.9) utilizada pelo operador ACG, era necessário restringir seu funcionamento considerando o tamanho da face $f$, para estabelecer sub-malhas iniciais "grandes". Desta forma e possível conseguir, por exemplo, o comportamento de particionamento mostrado na Figura 4.10
} 
onde $\operatorname{Vol}\left(\sigma_{i}\right)$ é o volume (área em 2D) da célula $\sigma_{i}, N_{r}(n+1)$ é definida como em (3.13) e

$$
N_{r_{V o l}}(n+1)=\sum_{i=1}^{N} p_{X_{i} \mid \mathbf{Y}}(r \mid \mathbf{y}, \theta(n)) \operatorname{Vol}\left(\sigma_{i}\right) \quad \forall r=1, \ldots, k
$$

A Figura 4.12 ilustra o funcionamento da abordagem PCAM. As Figuras Figura 4.12 (a),(b) e (c) apresentam o resultado do processo variando o peso da informação geométrica com valores de $\alpha=1, \alpha=2$ e $\alpha=3$, respectivamente. Como pode ser percebido informações geométricas podem ser de grande importância.

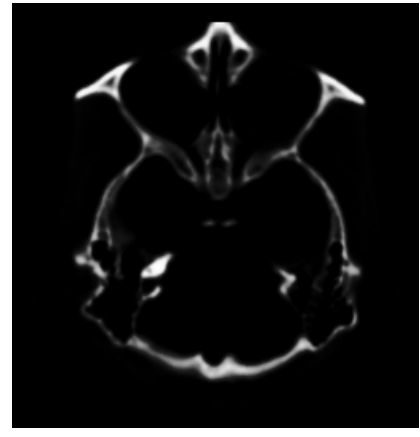

(a) Imagem original

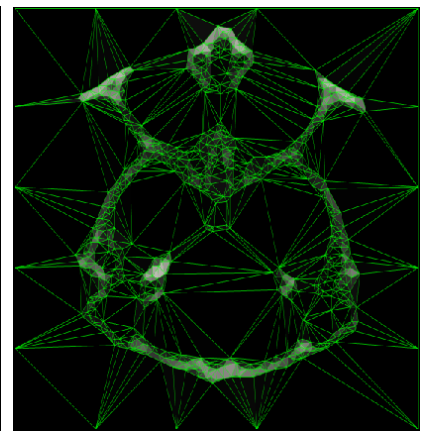

(b) Malha gerada

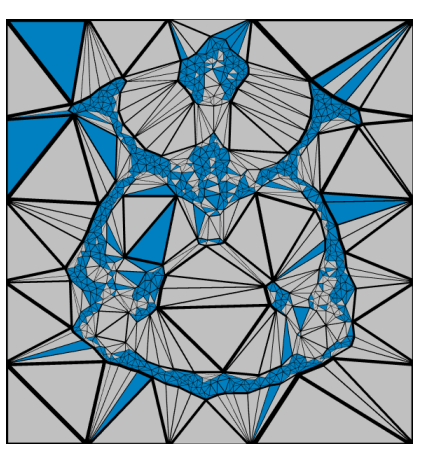

(c) $\alpha=0$

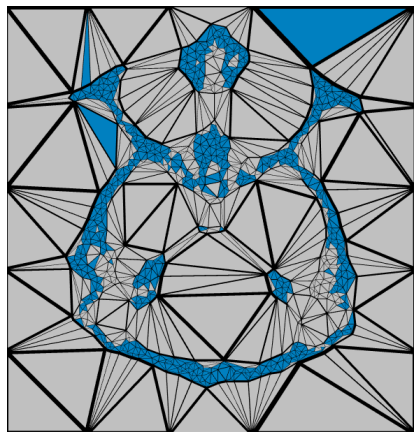

(d) $\alpha=1$

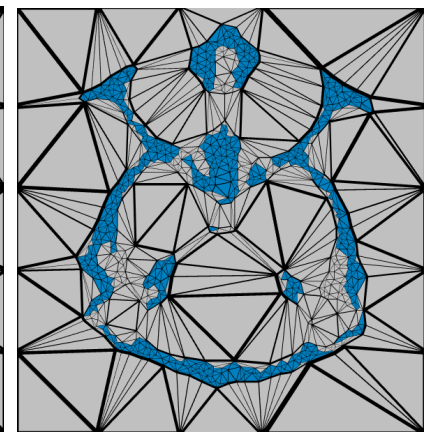

(e) $\alpha=2$ ou $\alpha=3$

Figura 4.12: Particionamento utilizando o operador a abordagem PCAM. Fatia de um crânio de macaco obtida por tomografia computadorizada 


\subsubsection{Detalhes e complexidade do processo de particionamento}

Os algoritmos para percorrer o interior de células $_{d}$, necessários para calcular padrões de cor dos elementos da malha, foram criados e otimizandos para serem robustos e muito flexíveis. Por outro lado, foram implementadas operações que interagem com estes procedimentos, como por exemplo, cálculo de média, histograma, área mais representativa, entre outras. Este fato, abre a possibilidade de testar e combinar novas idéias facilmente.

Sobre a complexidade no processo de segmentação, o maior esforço computacional é durante o cálculo de padrões de cor inicial. O algoritmo para percorrer todos os elementos da imagem (pixeis ou voxeis) contidos dentro de uma células ${ }_{d}$ é de comportamento linear. No entanto, quando realizado seqüencialmente estima-se que este tempo esteja ao redor de 95\% do tempo total do processo de particionamento. Por outro lado, o cálculo de padrão de cor de uma célula ${ }_{d}$ não depende dos cálculos realizados em outras células ${ }_{d}$. Sendo assim, este processo pode ser facilmente paralelizado acelerando fortemente o tempo deste cálculo.

Tendo em mãos os padrões de cor para cada célula $d$ da malha, os passos restantes do particionamento consideram unicamente estes valores numéricos em representação da porção de imagem dentro das células ${ }_{d}$. Este fato torna os cálculos de agrupamento muito mais ligeiros.

Os operadores de agrupamento ACV e ACG, podem ser facilmente implementados para ter um comportamento linear, pois tem o mesmo comportamento de algoritmos baseados em crescimento de região (Zucker, 2001; Hojjatoleslami e Kittler, 1998; Adams e Bischof, 1994). Adicionalmente, um fato importante é que durante as uniões realizadas por estes operadores, relações de vizinhança, primeiro entre células ${ }_{d}$, e logo entre sub-malhas, podem ser construídas e atualizadas. O cálculo de tais relações de vizinhança durante esta etapa é importante, pois acelera as operações realizadas pelo operador de redução de ruído ASmV.

Sobre o operador ASmV, podemos dizer que, a fila de prioridade, criada com a métrica da Equação 4.8, e que determina a relevância de cada sub-malha, pode ser atualizada para cada união em um tempo de $\log (n)$. Isto é, o tempo total, da atualização da fila de prioridade, durante as operações deste operador é proporcional a $n \log (n)$.

Sobre a complexidade associada às operações de consulta de vizinhança e agrupamento de sub-malhas, o cálculo para determinar se uma sub-malha é vizinha de uma outra pode ser realizado armazenando e atualizando estruturas que armazenam valores numéricos 
dentro de cada sub-malha. Pelos testes realizados, o tempo total de criação, consulta e atualização destas estruturas, durante as operações da particionamento, é muito baixo e oferece muita informação adicional. Além disso, a operação de fusão de duas sub-malhas pode ser realizado em tempo quase constante.

O operador ASmS, é um processo de natureza quadrática. No entando, normalmente, é utilizado para tratar um número reduzido de sub-malhas. Isto é, na prática este operador é utilizado para reduzir grupos de sub-malhas que contém, tipicamente, menos de 100 elementos. Ou seja, este operador é utilizado normalmente após uma redução drástica do número de sub-malhas efetuado por outros operadores.

Para determinar os elementos que compõem a barreira de restrição, todas as faces ${ }_{d}$ da malha são testadas, sendo este um processo de comportamento linear. Tendo identificado, na malha, todos os elementos da barreira de restrição, os cálculos realizados pelo operador $\mathrm{ACV}_{B R}$ para determinar se é possível ou não ultrapassar certas regiões pode ser implementado em tempo constante.

Finalmente, o algoritmo de particionamento por campos aleatórios Markovianos, possui um comportamento quadrático. No entanto, existem vários detalhes que poderiam ser otimizados para obter uma maior velocidade. Por exemplo, existe um número considerável de células ${ }_{d}$ que não mudam de rótulo desde as primeiras iterações até o final do algoritmo.

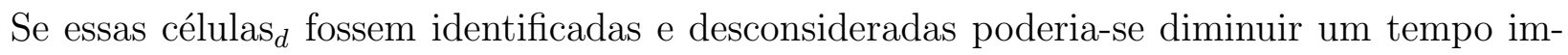
portante ao utilizar esta abordagem. Outro aspecto importante a considerar é que, durante as iterações do algoritmo, o posicionamento das células ${ }_{d}$ que mais mudam tem uma relação forte com os bordos das malhas. Isto é, fronteiras entre sub-malhas poderiam ser identificadas por combinações de operadores de agrupamento. Em tal contexto, a abordagem por campos aleatórios Markovianos poderia ser aplicada unicamente para suavizar fronteiras já definidas. Esse fato poderia acelerar e otimizar a utilização dessa abordagem estatística.

\subsection{Melhoramento de qualidade de malha}

A idéia deste último passo do algoritmo Imesh é incluir critérios de qualidade nos elementos de uma malha Delaunay gerada a partir de uma imagem, respeitando ao mesmo tempo, as fronteiras definidas durante o processo de particionamento de malha. 
Começaremos descrevendo na Seção 4.3.1 os detalhes e critérios utilizados no processo de refinamento de qualidade de malha Delaunay. Seguidamente, a Seção 4.3.2 explica como a rotulação de células ${ }_{d}$, definida durante o particionamento, pode ser mantida no final refinamento. Finalmente a Seção 4.3.3, discute sobre aspectos relacionados à complexidade do processo.

\subsubsection{Refinamento}

Considere $\rho$ como uma função que mede a qualidade de qualquer célula $\sigma_{i} \in M$. Utilizando a função $\rho$ pode-se estabelecer quais células ${ }_{d}$ de $M$ serão consideradas ruins durante o processo de refinamento. Por exemplo, pode-se considerar uma célula $\sigma_{d} \sigma_{i}$ como ruim quando $\rho\left(\sigma_{i}\right)>B$, onde $B$ é um limiar pré-estabelecido. Uma vez identificadas as células ${ }_{d}$ ruins da malha $M$, deve-se determinar em que ordem tais células ${ }_{d}$ serão processadas durante o refinamento. Para isto, cria-se uma lista $\delta$ de prioridade baseada na função $\rho$. Nessa lista as células ${ }_{d}$ com pior qualidade apareceram nos primeiros lugares. Utilizar esta ordem é importante pois, como sugerido no trabalho de Shewchuk (2004), no final do processo de refinamento, uma malha com uma quantidade menor de células ${ }_{d}$ é obtida. Tendo esta ordem em mãos, utilizamos a idéia básica dos algoritmos de refinamento de qualidade Delaunay, como explicado na Seção 3.2. Ou seja, inserir circuncentros de células $d$ ruins para eliminá-las.

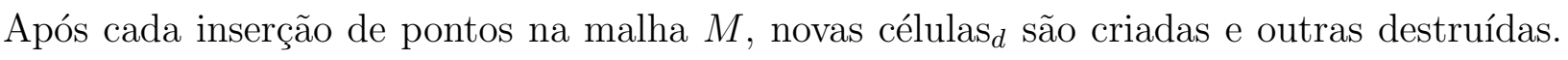
Assim, as células ${ }_{d}$ eliminadas, que estavam presentes na lista $\delta$ devem ser removidas e as

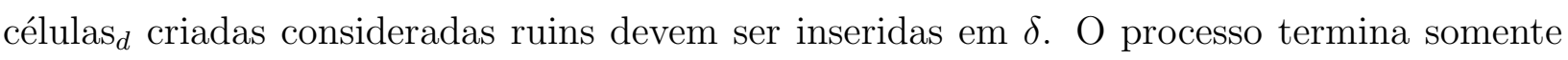
quando todas as células ${ }_{d}$ em $\delta$ forem eliminadas.

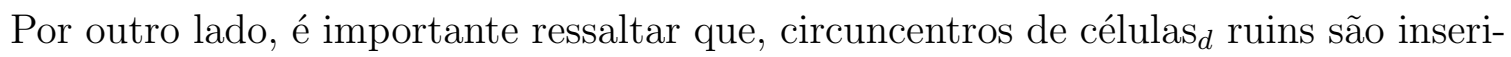

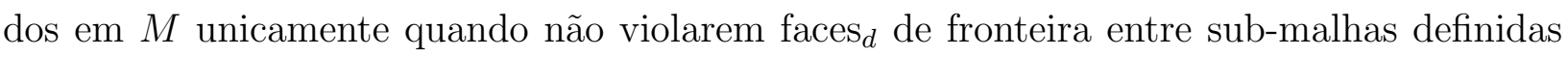
no processo de particionamento. No caso em que um circuncentro viola uma face ${ }_{d}$ de bordo, como sugerido nos algoritmo de refinamento Delaunay, esta pode ser sub-dividida inserindo o centro da mesma. A inserção deste tipo de pontos, quando o domínio de entrada possui ângulos pequenos, pode acarretar que o processo de refinamento não termine, como descrito na Seção 3.2.5. Adicionalmente um processo de tratamento de faces $_{d}$ de bordo deve também

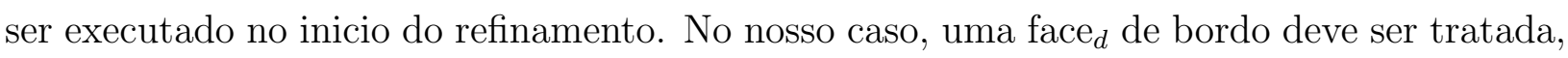
quando esta violada por algum outro ponto da triangulação ${ }^{9}$. Os critérios para determinar

\footnotetext{
${ }^{9}$ No nosso caso, após o particionamento, no existem faces $d$ que não apareçam na triangulação.
} 
quando uma face $_{d}$ de bordo é violada, e os processos aplicados para tratá-las, serão descritos separadamente para os algoritmo de duas e três dimensões. As idéias utilizadas para evitar que os algoritmos de refinamento, bidimensional e tridimensional, não terminem, e o critérios utilizados para determinar quando uma célula ${ }_{d}$ é considerada ruim serão também descritos separadamente, para duas e três dimensões.

Seja $c c_{i}$ o circuncentro de uma célula $\sigma_{i} \in M$ considerada ruim, e considere $f \in M$ como uma face ${ }_{d}$ de bordo na qual será executado o teste de violação. Considere também $r_{i}$, $d_{i}^{\text {min }}$ e $d_{i}^{\text {max }}$ como o circunraio, menor aresta, e a maior aresta de $\sigma_{i}$, respectivamente. Seja $B$ uma constante que expressa um limite inferior para determinar se uma aresta é considerada ruim, e $\Xi$ uma constante que limita o tamanho mínimo de uma aresta.

\section{- No caso bidimensional}

O algoritmo em duas dimensões, utiliza a função $\rho_{2}$ para determinar se uma célula $\sigma_{i}$ é considerada ruim é definida da seguinte maneira:

$$
\rho_{2}\left(\sigma_{i}\right)= \begin{cases}1 & \text { se } \frac{r_{i}}{d_{i}^{m i n}}>B \\ 0 & \text { caso contrário }\end{cases}
$$

Quando $\sigma_{i}$ é considerada ruim pela função $\rho_{2}, \sigma_{i}$ é inserida na lista de prioridade $\delta$, ordenada pelo fator, $\frac{r_{i}}{d_{i}^{\text {min }}}$. Por outro lado, a aresta $f$ é considerada violada pelo ponto $c c_{i}$, se e somente se, $c c_{i}$ está dentro, ou no bordo, do círculo diametral de $f$. Em duas dimensões, para tratar ângulos pequenos e evitar um refinamento infinito, foi adotada a abordagem proposta por Miller et al. (2003), descrita na Seção 3.2.5.

\section{- No caso tridimensional}

Com respeito ao algoritmo de refinamento em três dimensões, determina-se se uma célula $\sigma_{i} \sigma_{i}$ é, ou não, considerada ruim, utilizando a função $\rho_{3}$, definida da seguinte maneira:

$$
\rho_{3}\left(\sigma_{i}\right)= \begin{cases}1 & \text { se }\left|d_{i}^{\max }\right|>\Xi \mathrm{e} \frac{r_{i}}{d_{i}^{m i n}}>B \\ 0 & \text { caso contrário }\end{cases}
$$

Quando uma célula $\sigma_{i}$ é considerada ruim pela função $\rho_{3}, \sigma_{i}$ é inserida na lista $\delta$, ordenada pelo valor do comprimento da aresta máxima $\left|d_{i}^{\max }\right|$. Com respeito ao assunto 
de violação de faces $_{d}$ de bordo, o algoritmo tridimensional considera a face $d f$ como violada como descrito a seguir. Considere $p$ como o vértice em uma das duas células ${ }_{d}$ que contém $f$, oposto ao lado de $f$ onde se encontra o circuncentro $c c_{i}$. Sendo assim, a face $_{d} f$ é considerada violada por pelo ponto $c c_{i}$, se e somente se, $c c_{i}$ estiver localizado dentro, ou no bordo, da esfera formada pelos vértices de $f$ e o ponto $p$. Repare que utilizando tal critério de violação de faces $_{d}$, pode se evitar realizar o processo inicial de recuperação de faces $_{d}$ de bordo, pois pela natureza do critério não existem casos de violação de faces $_{d}$ na malha $M$, no começo do refinamento. Adicionalmente, este critério de violação evita refinamentos até o infinito, pois ângulos pequenos encontramse nos bordos, logo tais ângulos não serão movimentados. Por outro lado, a utilização da aresta máxima $\left|d_{i}^{\max }\right|$, de uma célula ${ }_{d}$ ruim $\sigma_{i}$, como critério de ordenação da lista de

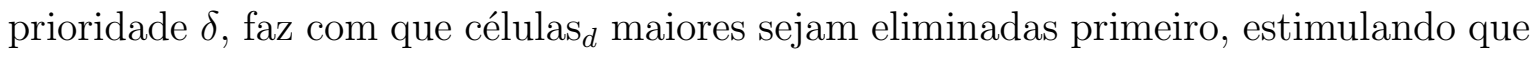
circuncentro sejam inseridos "longe" das fronteiras entre sub-malhas. Como a tendência de um algoritmo de refinamento Delaunay é originar arestas cada vez menores, a função $\rho_{3}$ também garante que o algoritmo termine pois, considera comparações entre arestas máximas com o limite inferior $\Xi$, para determinar se uma célula ${ }_{d}$ é ruim e por tanto inserida na lista $\delta$.

Por outro lado pode-se adaptar a função $\rho_{3}$ para que seja possível detectar tetraedros sliver. Isto é, pode-se substituir o fator $\frac{r_{i}}{d_{i}^{m i n}}$ por uma operação para detectar esse tipo de tetraedro ruim. Idéias de como implementar tal tipo de operação podem ser encontradas nos trabalhos de Shewchuk (2000b); Cheng et al. (2004); Pav e Walkington (2004).

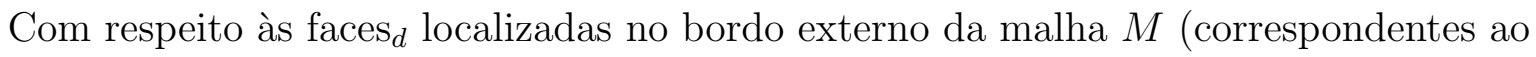
bordo externo da imagem de entrada), estas são tratadas da seguinte maneira. Quando

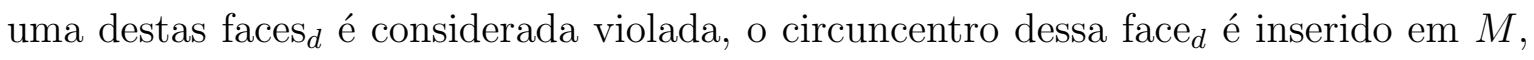
unicamente se este ponto não viola uma aresta do bordo externo da malha. Uma aresta do bordo externo é considerada violada quando sua esfera equatorial é invadida por um ponto. Em tal caso, o ponto médio da aresta de bordo invadida é inserido em $M$.

\subsubsection{Manutenção da rotulação}

A diferença do problema clássico de geração de malhas, descrito na Seção 3.2.1, no contexto do algoritmo Imesh, é que deve-se considerar o problema de como manter, no re- 
finamento, a rotulação estabelecida para as células ${ }_{d}$ durante o processo de particionamento. Isto é, após o processo de particionamento, cada célula ${ }_{d}$ possui um identificador que corresponde a uma sub-malha. Logo, quando um novo ponto é inserido durante o refinamento, células $_{d}$ são destruídas (perdendo informação) e outras são criadas (gerando informação não rotulada). O problema é então como manter esta rotulação atualizada. Este problema pode ser resolvido de uma forma muito simples e eficiente, como descrito a seguir.

Após o particionamento, grupos conexos de células ${ }_{d}$ com o mesmo rótulo são identificados, e para cada um destes grupos, uma semente é guardada com informação representando o grupo. Como o refinamento Delaunay, descrito na Seção anterior, garante que todas as fronteiras serão mantidas, a região compreendendo um grupo conexo inicial permanece intacta no final do processo. Assim, após o refinamento, a informação de cada semente pode ser expandida na componente conexa até atingir as fronteiras.

A Figura 4.13 apresenta exemplos de refinamentos bidimensionais utilizando as idéias descritas acima.

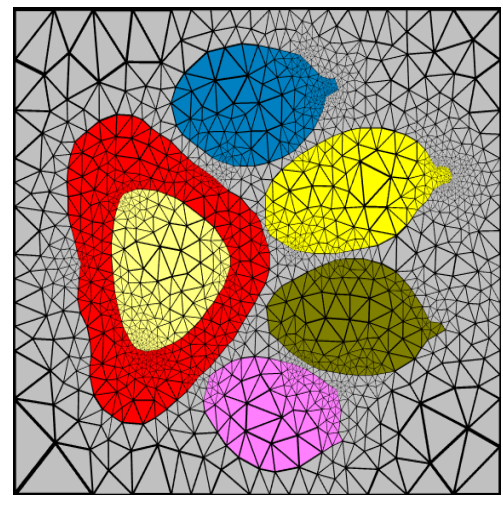

(a) Refinamento obtido a partir do particionamento da Figura 4.7 (d)

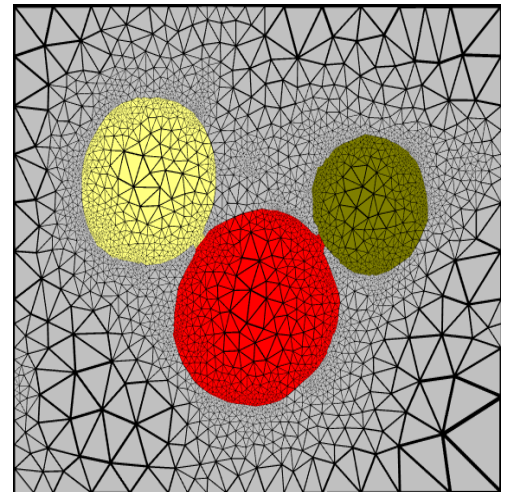

(b) Refinamento obtido a partir do particionamento da Figura 4.10 (d)

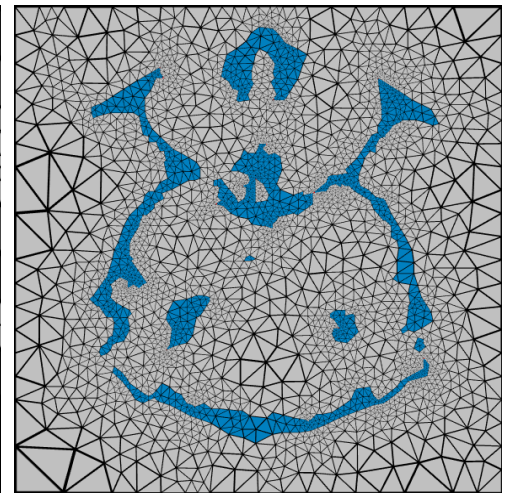

(c) Refinamento obtido a partir do particionamento da Figura 4.12 (e)

Figura 4.13: Etapa de refinamentod qualiade. Malhas obtidas com uma restrição de ângulo mínimo de $30^{\circ}$. 


\subsubsection{Detalhes e complexidade do processo de refinamento de qualidade de malha}

Os processos de refinamento Delaunay como descrito em Shewchuk (2004) e Shewchuk (1997), são de ordem $O\left(n^{2}\right)$, onde $n$ é o número de vértices inseridos da triangulação. Adicionalmente, deve ser considerado o tempo manutenção da lista de prioridade $\delta$. Esta ordenamento realizado na ordem de $m \log (m)$, onde $m$ é o número de células ${ }_{d}$ que foram consideradas com erro.

Os processos de obtenção das sementes iniciais e a expansão das sementes no final do processo, para manter a rotulação do particionamento, podem ser realizados em tempo linear. Além disso, utilizando esta idéia, ganha-se muita rapidez e simplicidade no algoritmo, pois, o refinamento realiza somente cálculos próprios do processo, sem ter que se preocupar em manter atualizada a rotulação do particionamento. Outro fato importante desta idéia é que pode ser estendida facilmente de duas para três dimensões. 


\section{Capítulo}

\section{Resultados}

Neste Capitulo, resultados da aplicação da técnica Imesh são apresentados e discutidos. A implementação do algoritmo Imesh foi realizada na linguagem de programação $C++$ (Stroustrup, 2000) e a biblioteca de geometria computacional CGAL (Fabri, 2001). A visualizações de resultados foi feitas utilizando a biblioteca de visualização cientifica Schroeder et al. (1998) e o software ParaView (2005). As imagens utilizadas, para os resultados do algoritmo bidimensional, foram extraídas dos seguintes lugares, Earth (2006); EMuseum (2006); Geographic (2006); Planet (2006); UT (2006); Bros (2006); Masks (2005). Os dados volumétricos de entrada para o algoritmo Imesh foram obtidos de Roettger (2006); Erlangen (2005); Stanford (2006). Todos os resultados apresentados foram executados em um computador com as seguintes características: Intel(R) Xeon CPU 3.2GHz, 4GB de RAM.

Apresentamos a seguir resultados bidimensionais e tridimensionais, separadamente.

\subsection{Resultados bidimensionais}

Apresentamos a seguir alguns exemplos do uso da técnica Imesh em duas dimensões. Focamos nossas discussões em comparações referentes ao comportamento e as características das triangulações obtidas quando a função erro pontual é alterada. Apresentamos ainda uma aplicação da técnica Imesh em geração de malhas para fins de simulação numérica, sendo essa, outra importante contribuição deste trabalho. 
Antes de começar a descrever os resultados obtidos é importante considerar o seguinte sobre as funções de erro pontual. A Função $\mathscr{E}_{i}$ foi criada para ser mais apropriada para representar imagens, por outro lado a função $\mathscr{E}_{v}$ foi pensada para realizar uma segmentação mais precisa. A função $\mathscr{E}_{g}$ possui características que podem ser utilizadas tanto para representar imagens quanto para criar malhas para fins de segmentação.

- Como mencionado anteriormente, dependendo da escolha da função de erro pontual, diferentes triangulações são obtidas. Fazendo uso de uma função de erro pontual de homogeneidade, por exemplo $\mathscr{E}_{v}$, obtém-se triangulações onde células $d$ permanecem, predominantemente, no interior dos objetos da imagem, possibilitando assim um melhor particionamento. Este fato é ilustrado na Figura 5.1. A Figura 5.1(b) mostra a segmentação manual da Figura 5.1(a), onde folhas, laranja e fundo da imagem foram separados. As Figuras 5.1(c) e 5.1(d) mostram as triangulações geradas pelo Imesh alterando-se apenas a função erro pontual. Na Figura 5.1(c) a função $\mathscr{E}_{v}$ foi empregada, sendo a função $\mathscr{E}_{i}$ utilizada na geração da triangulação em 5.1d). A fim de realizarmos uma comparação mais justa, forçamos o mesmo número de vértices em ambas as triangulações (1083 vértices). As Figuras 5.1(e) e 5.1(g) mostram as partições obtidas pelo método PCAM e POpA, tendo como base a triangulação da Figura 5.1(c). Tais segmentações são visivelmente melhores que as apresentadas nas Figuras 5.1(f) e 5.1(h), sendo que os mesmos algoritmos de particionamento, com os mesmos parâmetros empregados em 5.1(e) e 5.1(g), foram empregados, mas tomando como base a triangulação gerada com a função de erro pontual $\mathscr{E}_{i}$ mostrada na Figura 5.1(d).

A Tabela 5.1 apresenta uma comparação quantitativa do erro cometido nos particionamentos apresentados na Figura 5.1. Cada triangulação foi convertida em uma imagem percorrendo os pixels interiores a cada triângulo, sendo o valor do rótulo do triângulo atribuído como escalar para os pixels. As linhas da Tabela correspondem a porcentagem de pixels classificados errados nos particionamentos mostrados em 5.1(e) a 5.1(h), tendo como base a segmentação manual apresentada na Figura 5.1(b). Como esperado, melhores resultados foram obtidos com a triangulação gerada a partir da função de erro pontual $\mathscr{E}_{g}$, tendo a técnica POpA atingido o menor erro de classificação.

- Apresentamos a seguir uma comparação das malhas geradas com as funções de erro pontual $\mathscr{E}_{g}$ e $\mathscr{E}_{i}$ quando empregadas para modelagem de imagem. Nesse contexto, a 


\begin{tabular}{|l|c|}
\hline Particionamento & Erro de Classificação \\
\hline $\mathscr{E}_{v}$ PCAM & $6.23 \%$ \\
\hline $\mathscr{E}_{v}$ POpA & $5.54 \%$ \\
\hline $\mathscr{E}_{i}$ PCAM & $8.79 \%$ \\
\hline $\mathscr{E}_{i}$ POpA & $20.93 \%$ \\
\hline
\end{tabular}

Tabela 5.1: Porcentagem de pixels classificados errados no particionamento.

idéia é produzir malhas que minimizem o erro de interpolação entre a imagem original e a imagem produzida a partir da malha, interpolando linearmente o nível de cinza atribuído aos vértices de um triângulo para os pixels internos desse triângulo. A Figura 5.2 apresenta os resultados qualitativos desta comparação. As Figuras 5.2(b) e 5.2(c) mostram as malhas geradas a partir da imagem mostrada na Figura 5.2(a) empregando as funções de erro pontual $\mathscr{E}_{g}$ e $\mathscr{E}_{i}$, respectivamente. A Figura 5.2(d) apresenta a malha gerada por uma técnica de modelagem de imagens baseada em quadtree ?. A Técnica de modelagem de imagens baseada em quadtree busca subdividir os nós da árvore onde o erro de interpolação é mais acentuado, realizando uma triangulação dos nós folha no final do processo. As três triangulações apresentadas nas Figuras 5.2(b), 5.2(c) e 5.2(d) foram geradas com aproximadamente o mesmo número de vértices (6180 vértices, correspondendo a aproximadamente $2.36 \%$ dos pixels da imagem original, cuja dimensão é $512 \times 512$ ). As Figuras 5.2(e), 5.2(f) e 5.2(g), apresentam as imagens obtidas por interpolação linear a partir dos dados nos vértices das triangulações mostradas nas Figuras 5.2(b), 5.2(c) e 5.2(d), respectivamente. Pode-se notar, mesmo visualmente, que a malha gerada com a função de erro pontual $\mathscr{E}_{i}$ produz o melhor resultado dentre as demais.

A Tabela 5.2 comprova a observação acima, apresentando medidas quantitativas dos erros obtidos. Note que a técnica Imesh com a função de erro pontual $\mathscr{E}_{i}$ produziu resultados superiores a quadtree, que é uma abordagem bastante difundida e empregada em modelagem de imagens.

\begin{tabular}{|l|c|}
\hline Imagem interpolada & Erro de médio de interpolação \\
\hline Imagem Figura 5.2(e) & 16.50 \\
\hline Imagem Figura 5.2(f) & 8.38 \\
\hline Imagem Figura 5.2(g) & 9.70 \\
\hline
\end{tabular}

Tabela 5.2: Erro médio das interpolações. 
Um fato interessante ocorre quando as imagens são geradas com uma interpolação de ordem zero, ou seja, a média dos níveis de cinza em um triângulo é atribuida a todos os seus pixels interiores. Com esse tipo de interpolação, a imagem produzida a partir da malha gerada com a função de erro pontual $\mathscr{E}_{g}$ torna-se mais nítida que a imagem gerada com a função $\mathscr{E}_{i}$, como pode ser observado na Figura 5.3 (compare os detalhes dos olhos e boca nas Figuras 5.3(a) e 5.3(b)).

- Apresentamos a seguir a interação entre alguns dos operadores apresentados dentro de estratégia de particionamento POpA. A Figura 5.4 (a) apresenta quatro objetos com padrões de cor similares, e a Figura 5.4 (b) apresenta a malha gerada para este exemplo utilizando a função $\mathscr{E}_{v}$. A Figura 5.4 (c) mostra um exemplo que utiliza apenas critérios de similaridade de padrões de cor para realizar o particionamento. Logo, a Figura 5.4 (d) apresenta como a interação dos operadores ACV e ASmV podem isolar objetos separados com padrões de cor similares. Porém, tal interação não é capaz de separar objetos que estão juntos e possuim padrões de cor similares. Finalmente a Figura 5.4 (e), mostra como a inclusão do operador que considera critérios geométricos ACG pode resolver o problema.

- Os seguintes resultados apresentam malhas onde critérios de qualidade são considerados. Todos os exemplos utilizam uma restrição de ângulo mínimo de $30^{\circ}$.

A figura 5.5 apresenta todas as etapas do algoritmo, desde a construção da malha (figura 5.5b)), passando pelo particionamento (figura 5.5c)) até o refinamento com qualidade (figura 5.5d)). As Figuras 5.6(a) e (b) ilustram a aplicação do Imesh, juntamente com a etapa de refinamento, em uma imagem médica. Note que mesmo estruturas finas foram capturadas pelo algoritmo. Outro exemplo desse tipo de aplicação é mostrada nas Figuras 5.6(c) e (d), onde mapeamento de textura e informação de elevação foram incorporados à malha gerada a partir de uma imagem de satélite do Lago Superior.

A tabela 5.3 mostra os tempos de processamento envolvidos na construção das malhas apresentadas nas Figuras 5.5, 5.6. Note que o algoritmo, no caso 2D, é bastante rápido, gastando aproximadamente 2.5 segundos para a maior imagem (Lago Superior).

- Terminamos esta seção com a apresentação na Figura 5.7 de uma aplicação onde uma deformação elástica é realizada sobre a malha gerada a partir da imagem da Figura 5.7 


\begin{tabular}{|c|c|c|c|c|c|}
\hline & $\begin{array}{c}\text { Dimensão } \\
\text { da imagem }\end{array}$ & $\begin{array}{c}\text { Construção } \\
\text { triangulação }\end{array}$ & $\begin{array}{c}\text { Particio- } \\
\text { namento }\end{array}$ & $\begin{array}{c}\text { Refinamento } \\
\text { qualidade }\end{array}$ & Total \\
\hline Peça Motor & $256 \times 256$ & 0.30 & 0.18 & 0.43 & 0.91 \\
\hline Aneurisma & $474 \times 794$ & 0.62 & 0.03 & 1.98 & 2.63 \\
\hline Lago Superior & $1144 \times 570$ & 0.98 & 0.48 & 0.96 & 2.42 \\
\hline
\end{tabular}

Tabela 5.3: Tempos computacionais (em segundos)

(a). A Figura 5.7 (b) apresenta a malha sobreposta à imagem. O modelo elástico é construído considerando os vértices e arestas da malha como partículas com massa e molas respectivamente. Desta forma, a simulação do movimento de malha é realizada através da resolução de equações diferenciais ordinárias de primeira ordem. Foram definidos alguns conjuntos de pontos de controle nos vértices da malha, para obter diferentes deformações da Figura original. Estes pontos podem ser observados na Figura 5.7 (c). Cada mola inicialmente tem um comprimento de repouso. Logo, quando vértices são deslocados surgem forças cujas intensidades são dadas pela Lei de Hook, $F=k . x$, onde $k$ é a constante elástica de mola e $x$ e o deslocamento das partículas em relação ao comprimento de repouso. As Figuras de Figura 5.7 (d) até Figura 5.7 (k) apresentam algumas deformações realizadas, pela movimentação dos conjuntos de pontos de controle.

- Otras malhas obtidas pelo algoritmo Imesh bidimensional podem ser apreciadas na Figura 5.8.

\subsection{Resultados tridimensionais}

Esta seção apresenta como toda a teoria e resultados envolvidos com o algoritmo Imesh bidimensional podem ser naturalmente estendidos para o caso tridimensional.

- Começamos apresentando um exemplo da aplicação de critérios geométricos no particionamento tridimensional. A Figura 5.9 (a) apresenta um corte de um coelho de porcelana contido numa imagem tridimensional. A Figura 5.9 (b) mostra o rendering volumétrico da malha obtida pela função $\mathscr{E}_{g}$. Como pode ser observado, o nariz do coelho esta encostado em outro objeto (com padrões de cor similares) contido na imagem. Este fato dificulta o isolamento do coelho durante o particionamento, como mostra a Figura 5.9 (c). No entanto utilizando critérios geométricos é possível separar os objetos da imagem corretamente. 


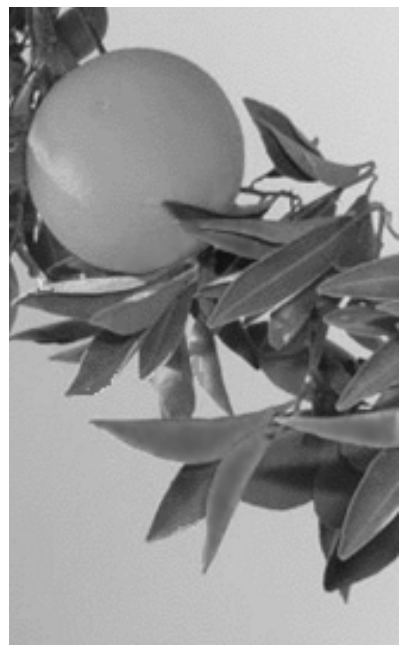

(a) Imagem original

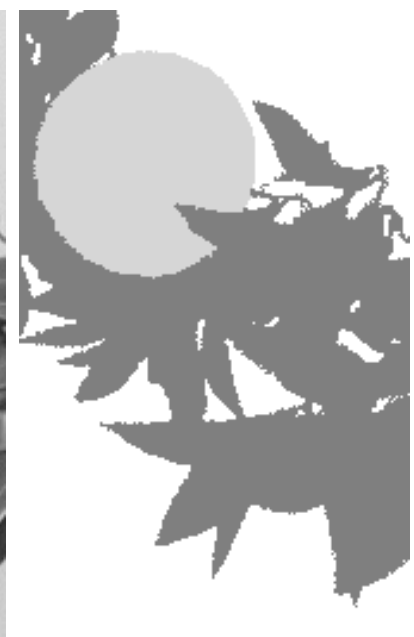

(b) Segmentação manual

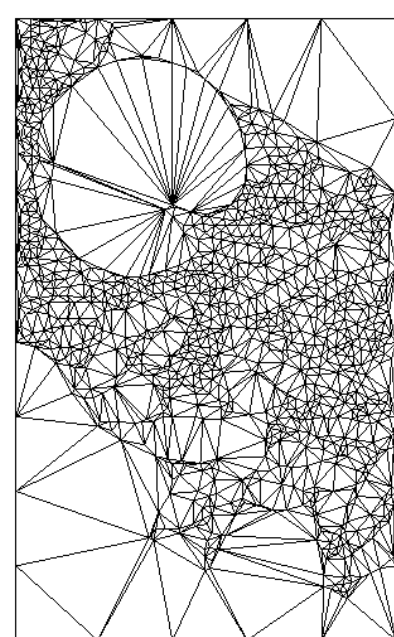

(c) Malha obtida com função de erro pontual $\mathscr{E}_{v}$

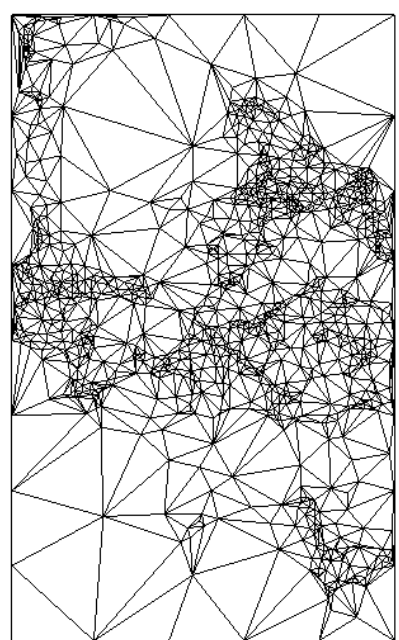

(d) Malha obtida com função de erro pontual $\mathscr{E}_{i}$

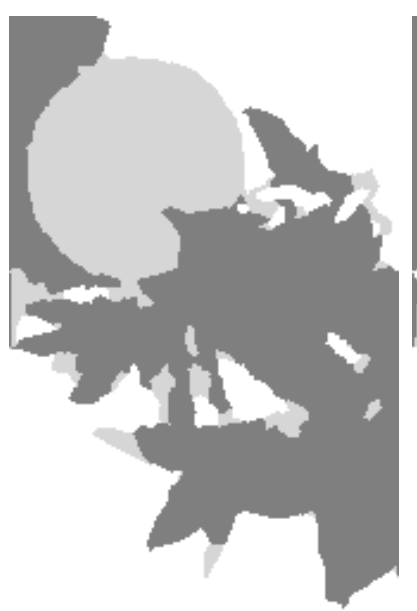

(e) Particionamento por PCAM $\alpha=3$ da malha da Figura (c)

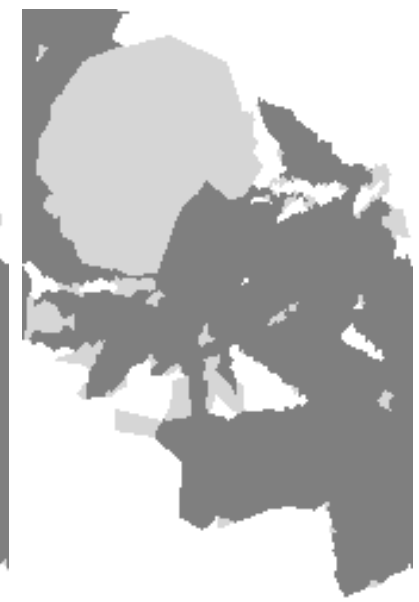

(f) Particionamento por PCAM $\alpha=3$ da malha da Figura (d)

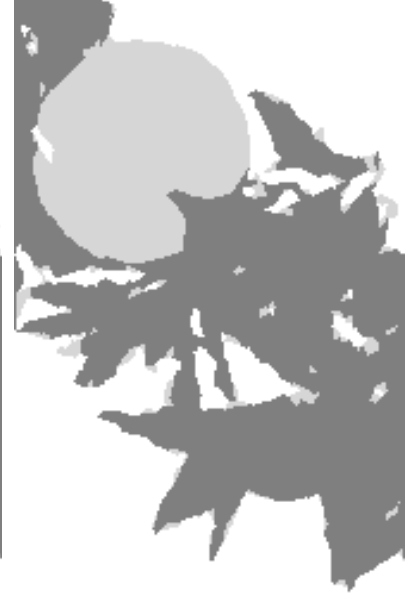

(g) Particionamento por $\mathrm{ACV}+\mathrm{ASmV}$ (50)+ASmS (3), da malha da Figura (c)

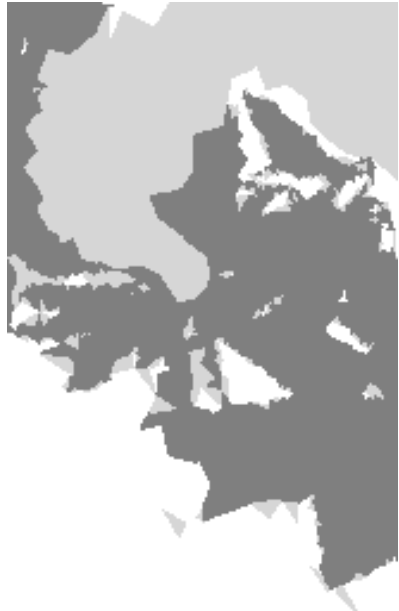

(h) Particionamento por $\mathrm{ACV}+\mathrm{ASmV}$ (50)+ASmS (3), da malha da Figura (d)

Figura 5.1: Comparação dos particionamentos obtidos pelas abordagens PCAM e POpA, em malhas obtidas por funções de erro pontual de interpolação e de homogeneidade.

- O seguinte exemplo mostra um caso onde a não utilização da idéia de barreira de 
restrição, durante o particionamento, pode causar resultados não desejados. Isto é, o ruído presente nas células ${ }_{d}$ da malha, no caso tridimensional, é mais intenso e pode originar situações inesperadas. A Figura 5.10 (a) mostra uma seção planar de uma imagem médica de um aneurisma, e a Figura 5.10 (b) apresenta o rendering volumétrico da malha gerada utilizando a função de erro pontual $\mathscr{E}_{v}$. Logo, a Figura 5.10 (c) mostra o resultado obtido sem considerar uma barreira de restrição. Finalmente, a Figura 5.10 (d) apresenta como este fenômeno pode ser controlado considerando o operador de barreira de restrição BR.

- O seguinte exemplo apresenta a utilidade da abordagem de particionamento PCAM. As Figuras 5.11(a) e (b) mostram um corte do volume de dados e o rendering volumétrico da malha gerada, respectivamente. A Figura 5.11 (c) apresenta um particionamento pela abordagem POpA sem a utilização de barreira de restrição. Logo, a Figura 5.11 (d) mostra como esta situação pode ser melhorada utilizando o particionamento PCAM.

- Apresentamos também a habilidade da técnica Imesh para gerar um particionamento com uma quantidade alta de sub-malhas. A Figura 5.12, mostra dois exemplos desse fato, detalhando para cada caso, um corte do volume de entrada, a visualização da malha via rendering volumétrico e o resultado do processo de particionamento, respectivamente. Repare que muitas das sub-malhas separadas possuem os mesmos padrões de cor na imagem de entrada.

- A seguir, apresentamos exemplos da etapa de refinamento de qualidade tridimensional. A Figura 5.13 (a) mostra o rendering volumétrico da malha gerada pelo operador $\mathscr{E}_{v}$, para o volume de dados chamado Buddha. A Figura 5.13 (b) apresenta o particionamento obtido. Nas Figuras 5.13(b) e (c) podem ser observados detalhes do resultado do processo de refinamento de qualidade. As Figuras 5.14 e 5.15 apresentam outros exemplos de refinamento tridimensional, com a mesma idéia. A Tabela 5.4 apresenta tempos das etapas do algoritmo Imesh para estes três últimos exemplos. A tabela mostra também a quantidade de tetraedros e pontos gerados no processo.

- Finalmelmente as Figuras 5.16 e 5.17 apresentam outros particionamentos obtidos e visualizações via rendering volumétrico, respectivamente. 


\begin{tabular}{|c|c|c|c|c|c|c|c|}
\hline & $\begin{array}{c}\text { Dimensão } \\
\text { da imagem }\end{array}$ & $\begin{array}{c}\text { Número } \\
\text { Pontos }\end{array}$ & $\begin{array}{c}\text { Número } \\
\text { células }_{d}\end{array}$ & $\begin{array}{c}\text { Construção } \\
\text { triangulação }\end{array}$ & $\begin{array}{c}\text { Particio- } \\
\text { namento }\end{array}$ & $\begin{array}{c}\text { Ref. } \\
\text { qual. }\end{array}$ & Total \\
\hline Buddha & $256 \times 512 \times 256$ & 59764 & 361129 & 1391.4 & 12.6 & 921.6 & 2325.6 \\
\hline Engine & $256 \times 256 \times 110$ & 26275 & 147488 & 625.9 & 5.34 & 164 & 795.14 \\
\hline Porsche & $599 \times 1023 \times 347$ & 286708 & 1856221 & 5122 & 51.68 & 4440 & 9613.76 \\
\hline
\end{tabular}

Tabela 5.4: Tempos computacionais para o algoritmo tridimensional(em segundos) 


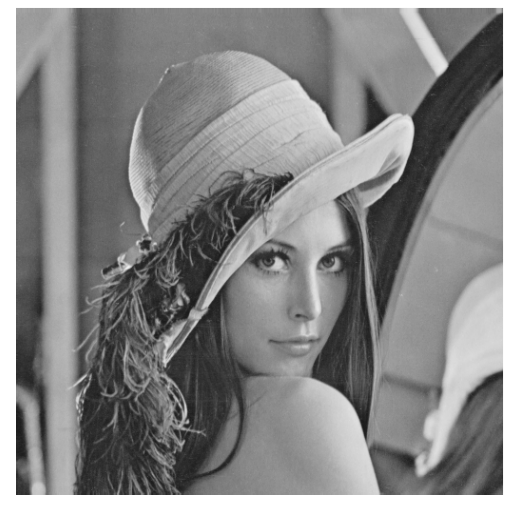

(a) Imagem original

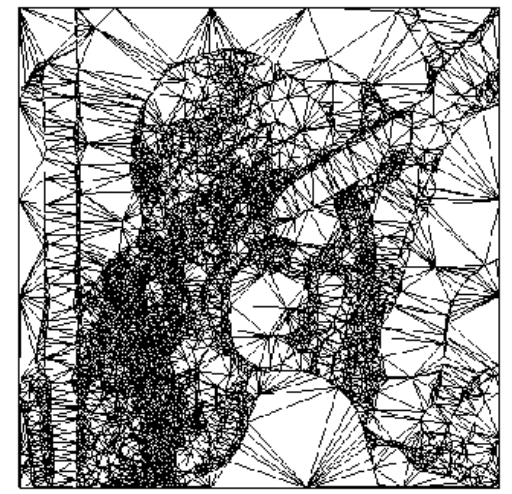

(b) Malha obtida com função de erro pontual $\mathscr{E}_{g}$

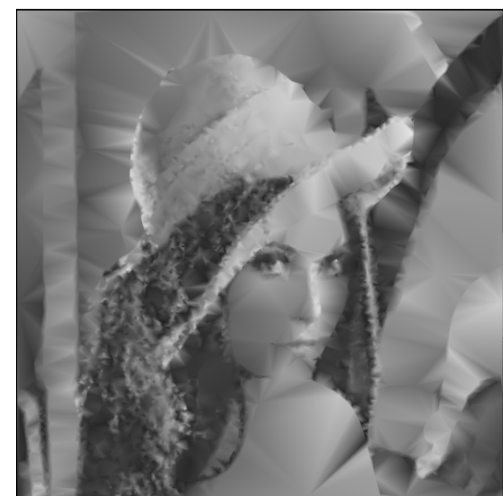

(e) Imagem interpolada a partir da malha (b)

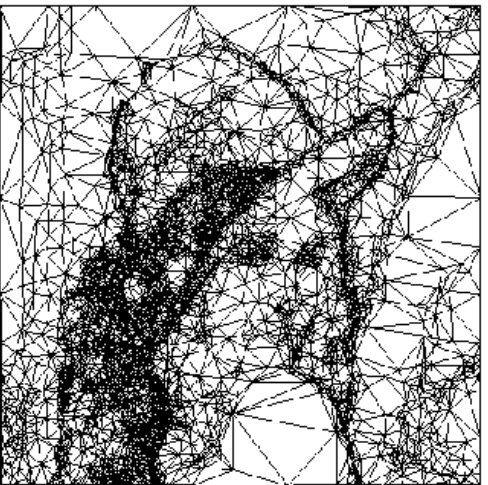

(c) Malha obtida com função de erro pontual $\mathscr{E}_{i}$

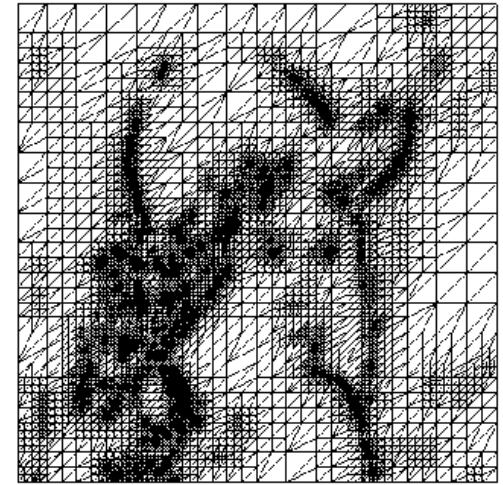

(d) Malha obtida por quadtree

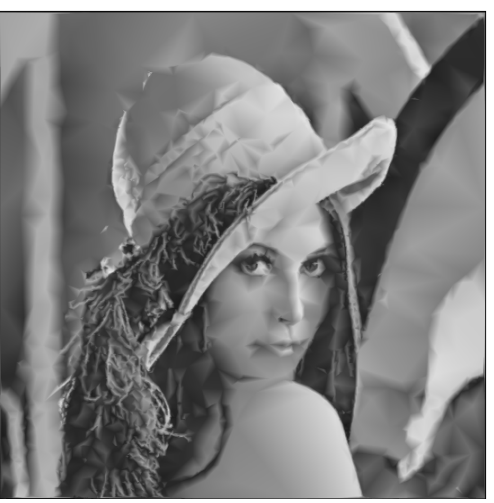

(f) Imagem interpolada a partir da malha (c)

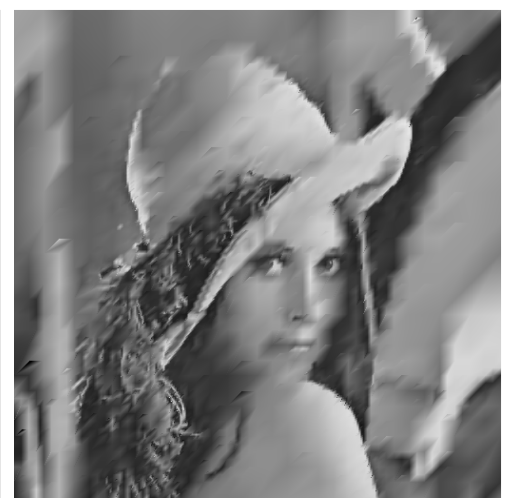

(g) Imagem interpolada a partir da malha $(\mathrm{d})$

Figura 5.2: Comparação dos erros de interpolação. 

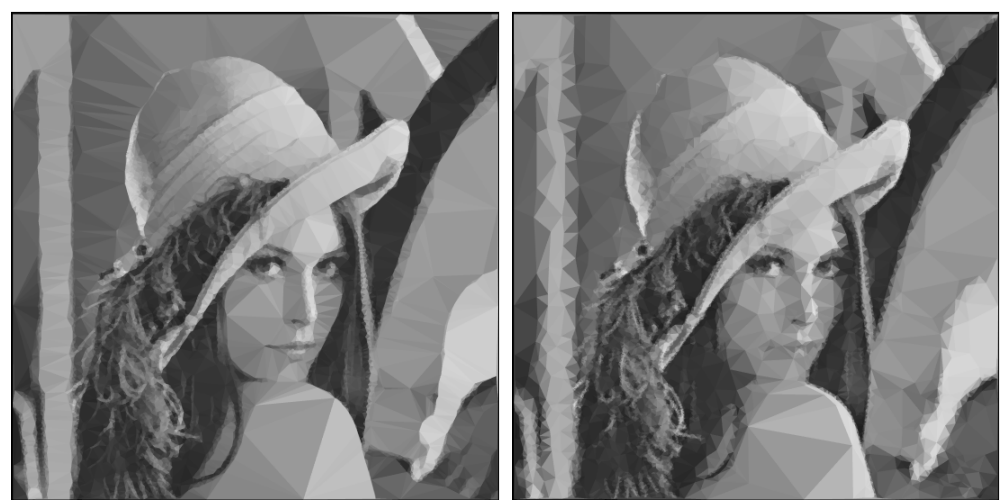

(a) Imagem interpolada

(b) Imagem interpolada a partir da malha da Figura 5.2(b)

a partir da malha da Figura 5.2(c)

Figura 5.3: Imagens geradas com interpolação de ordem zero. 


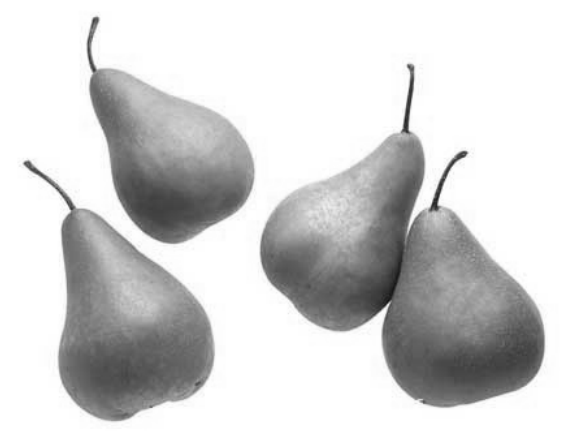

(a) Imagem original

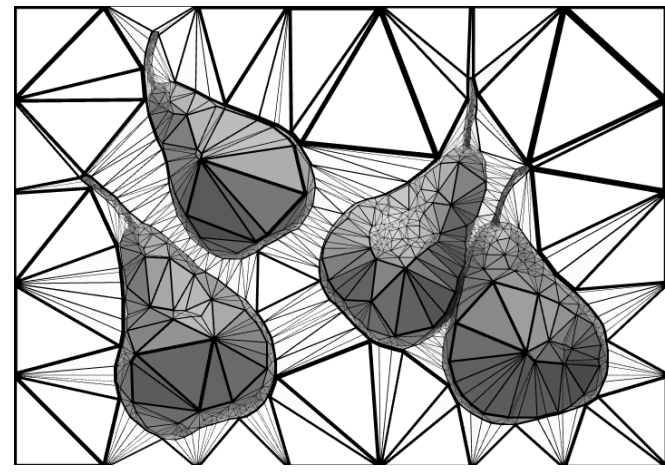

(b) Malha gerada, com $\mathscr{E}_{v}$, representada por padrões de cor (media)

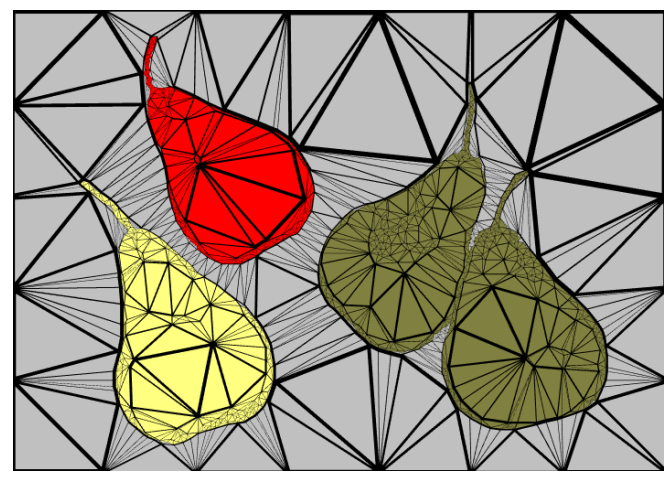

(d) Particionamento ACV + ASmV (4)

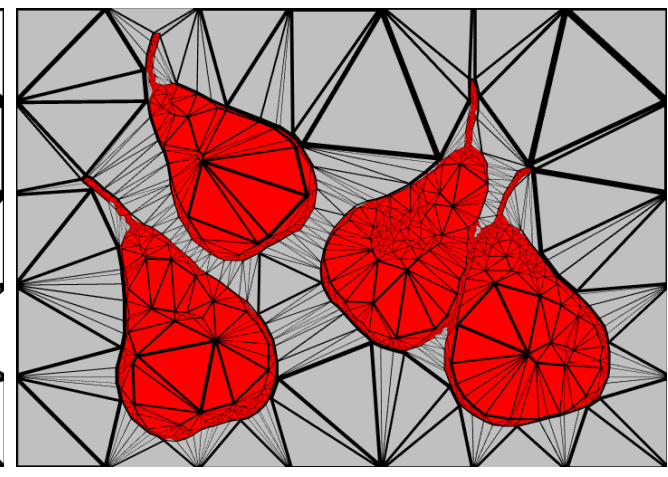

(c) Particionamento baseado somente em similaridade de padrões de cor.

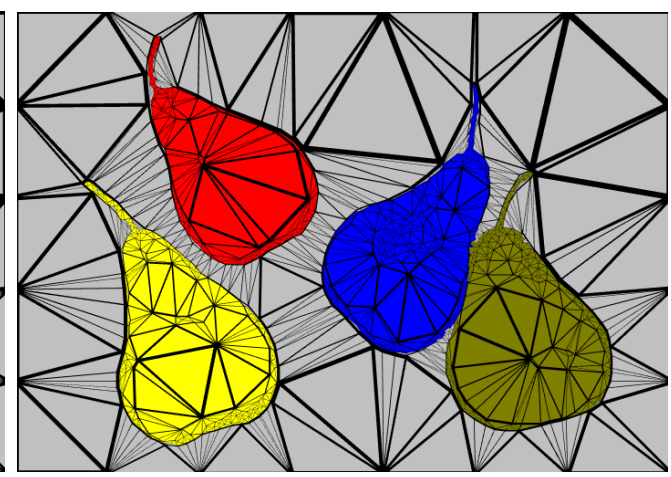

(e) Particionamento ACG (13) + ACV $+\mathrm{ASmV}(4)$

Figura 5.4: Interação entre alguns dos operadores da estratégia POpA e importância da geometria como critério de particionamento. 


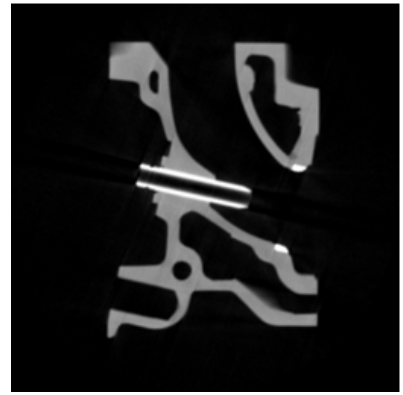

(a) Imagem de um corte em uma peça mecânica

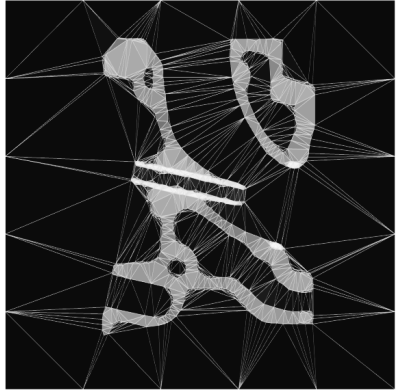

(b) Construção da malha, $\mathscr{E}_{g}$

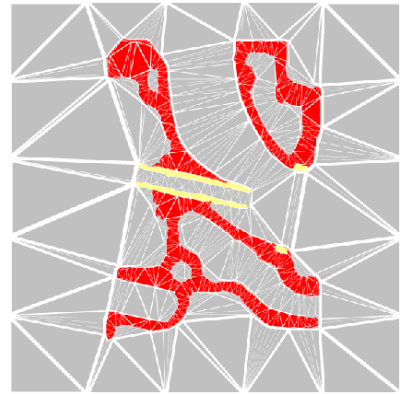

(c) Particionamento em 3 submalhas, $\mathrm{ACV}+\mathrm{ASmV}$ $(30)+\operatorname{ASmS}(3)$

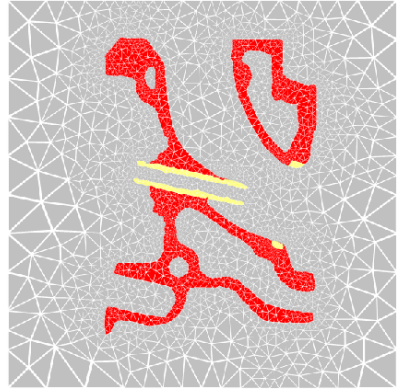

(d) Refinamento com qualidade

Figura 5.5: Processo completo do algoritmo Imesh bidimensional. 


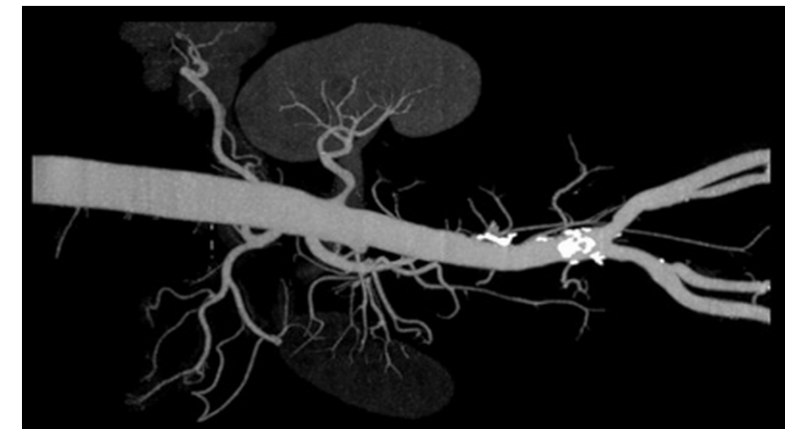

(a) Aneurisma

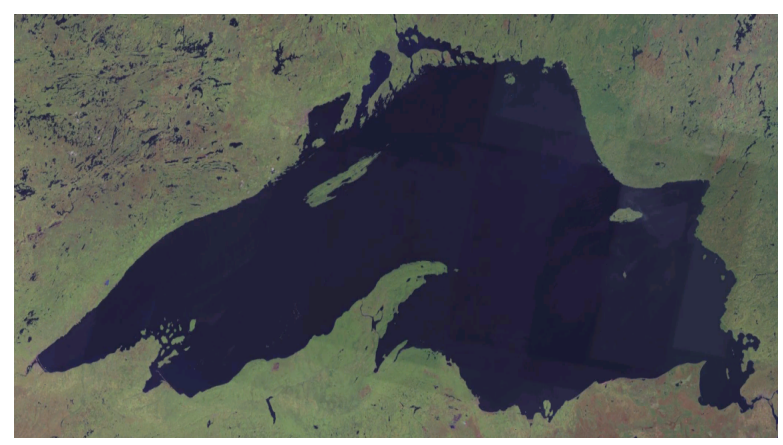

(c) Lago Superior

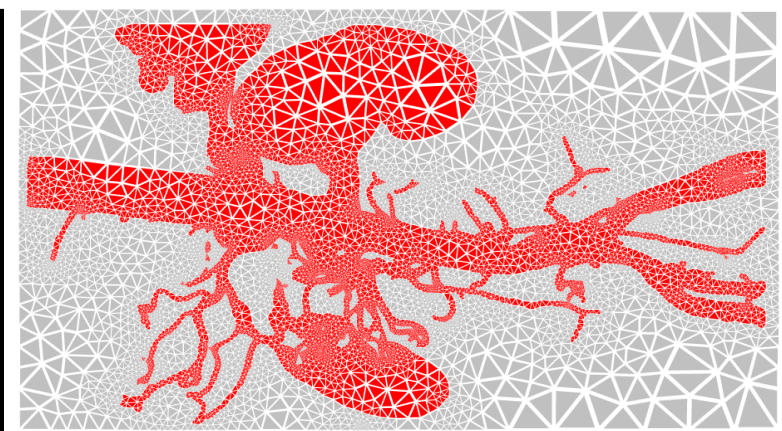

(b) Malha final, $\mathscr{E}_{g}+\mathrm{ACV}+\mathrm{ASmV}$ (17) + ASmS (2)

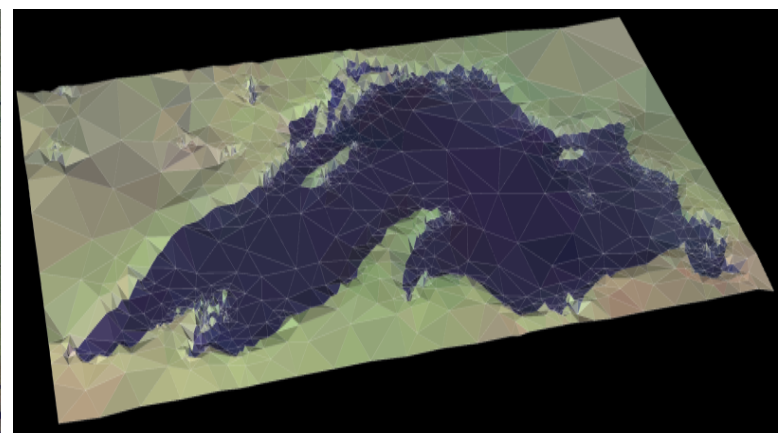
(d) Malha final, $\mathscr{E}_{g}+\mathrm{ACV}+\mathrm{ASmV}$
$(30)+\mathrm{ASmS}(2)$

Figura 5.6: Gerações de malha de qualidade. 


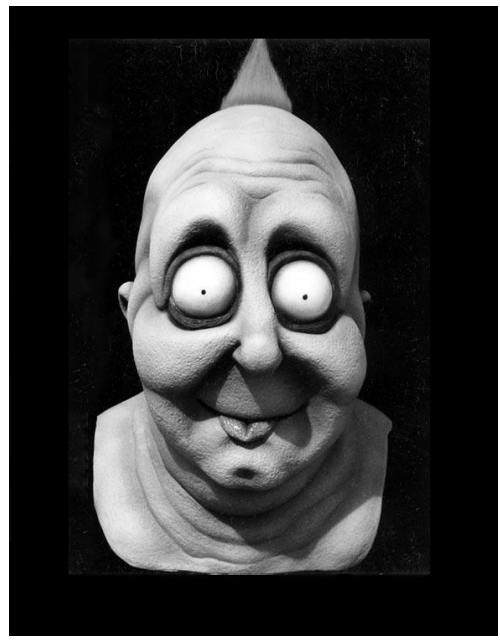

(a) Imagem original

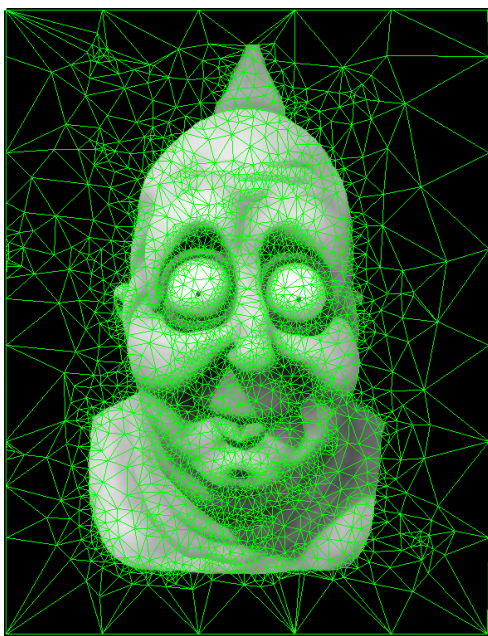

(b) Malha gerada com a função $\mathscr{E}_{g}$, Número de pontos $=3569$.

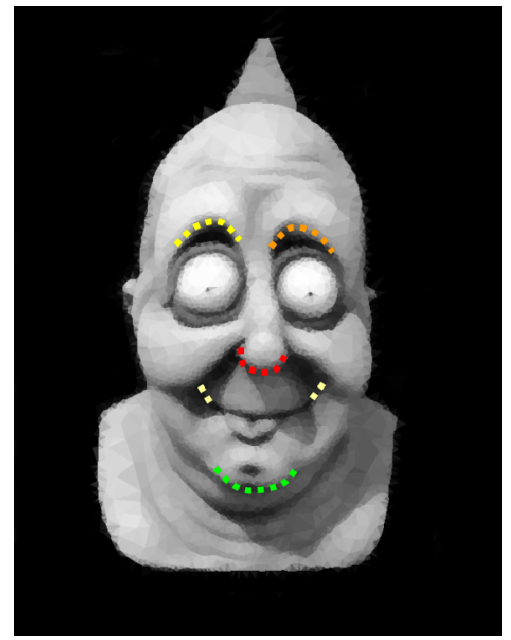

(c) Malha representado a imagem, com alguns conjuntos de pontos de controle.

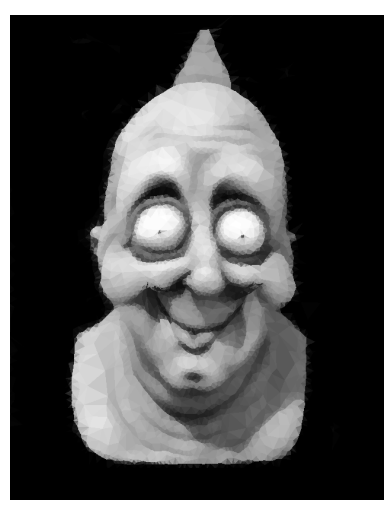

(d)

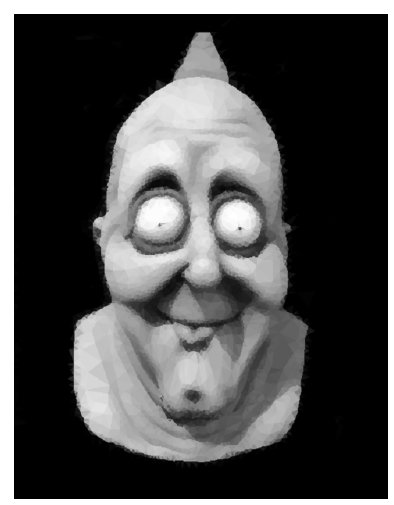

(h)

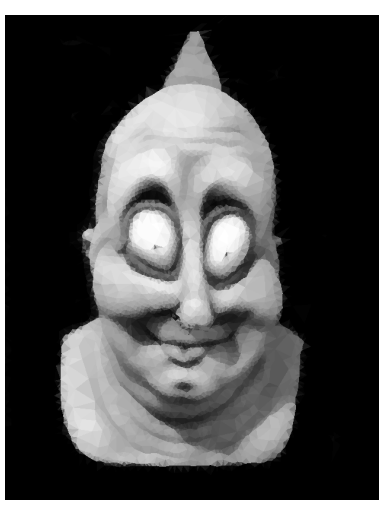

(e)

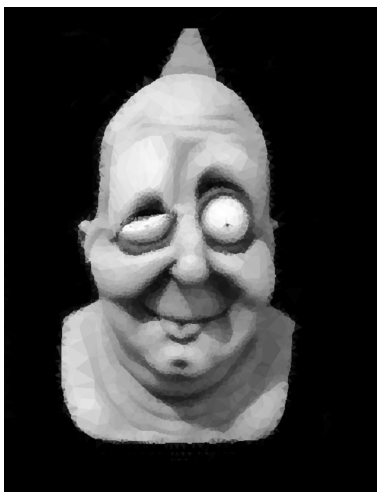

(i)

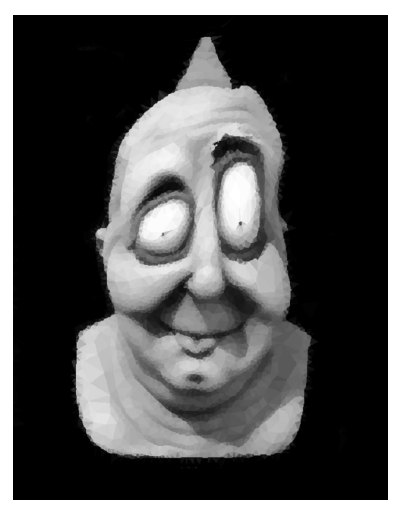

(f)

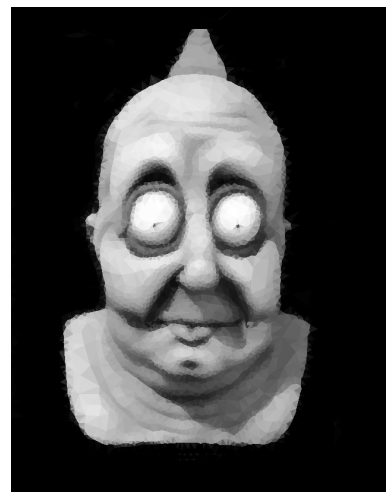

(j)

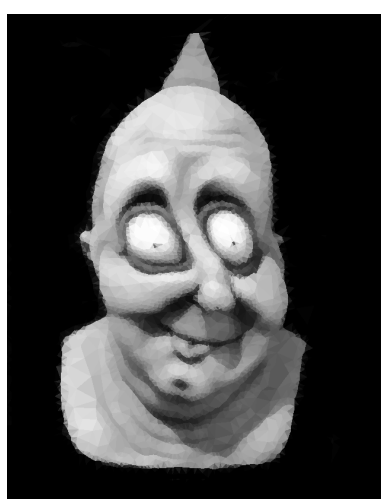

(g)

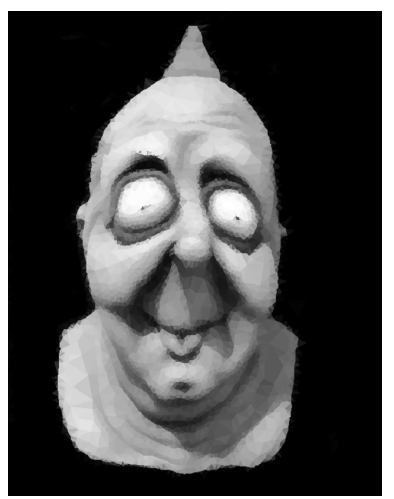

(k)

Figura 5.7: Teste de deformação utilizando malhas elásticas, a partir de uma imagem bidimensional. 


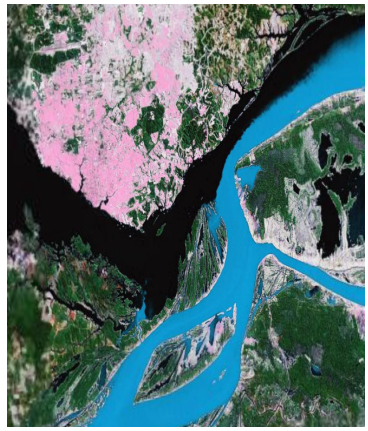

(a) Rio negro e solimões

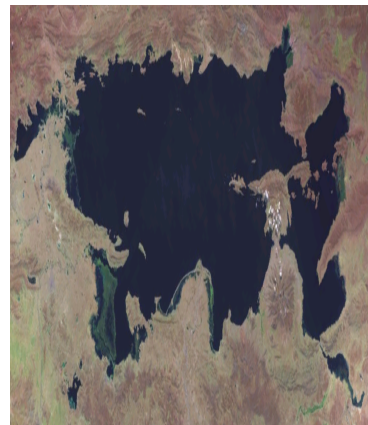

(e) Lago titicaca

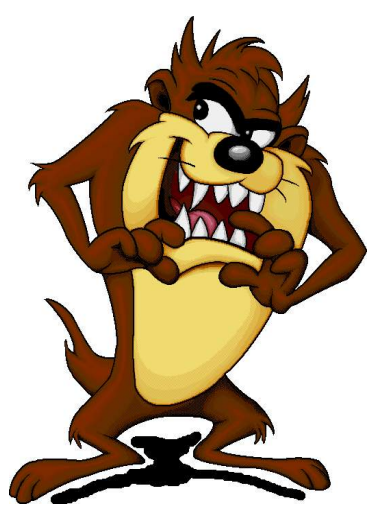

(i) Taz

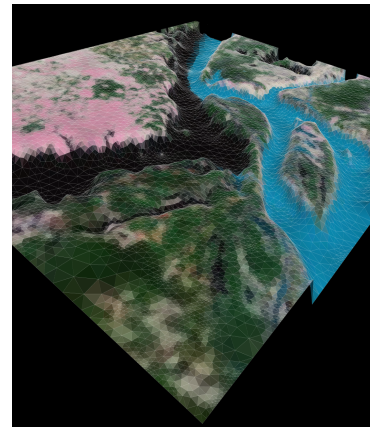

(b) Malha gerada

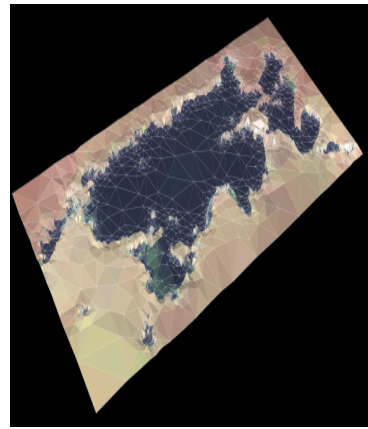

(f) Malha gerada

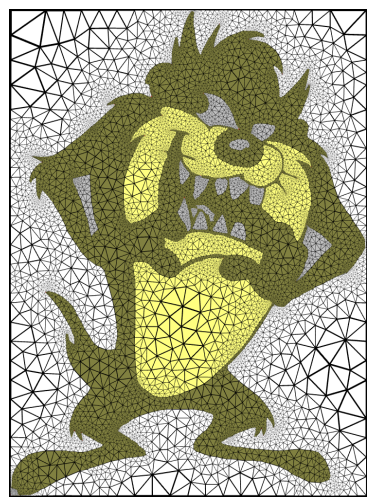

(j) Malha gerada

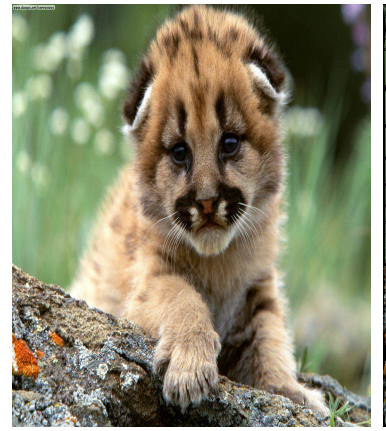

(c) Leõncinho

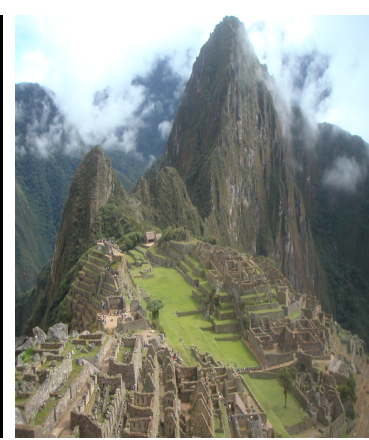

(g) Machu Picchu

(h) Malha gerada

(d) Malha gerada
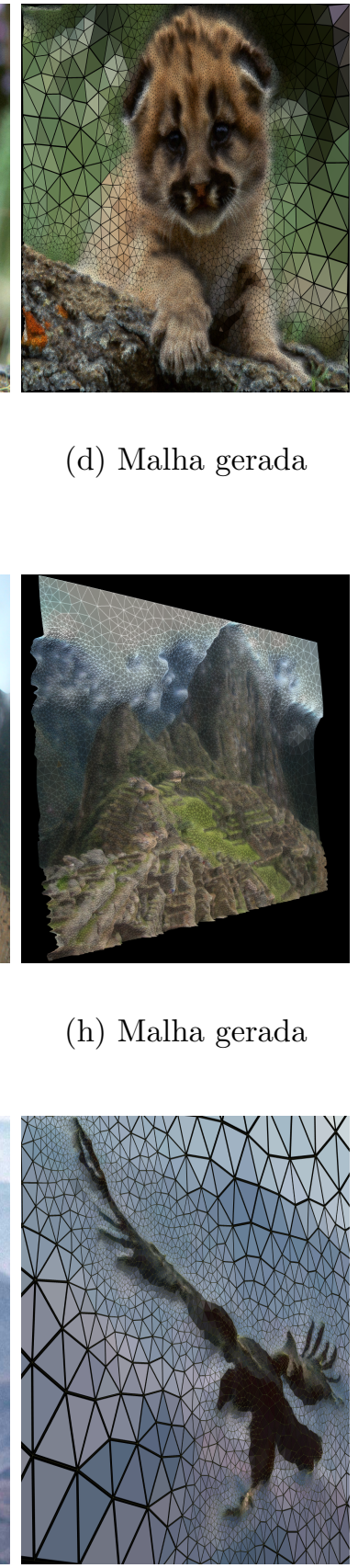

(k) Condor

(1) Malha gerada

Figura 5.8: Outros resultados bidimensionais obtidos. 


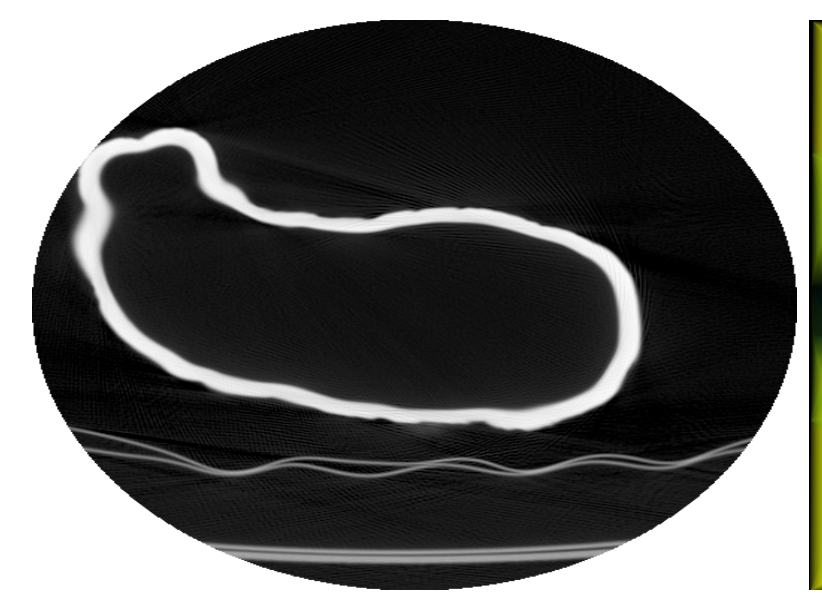

(a) Corte da imagem original

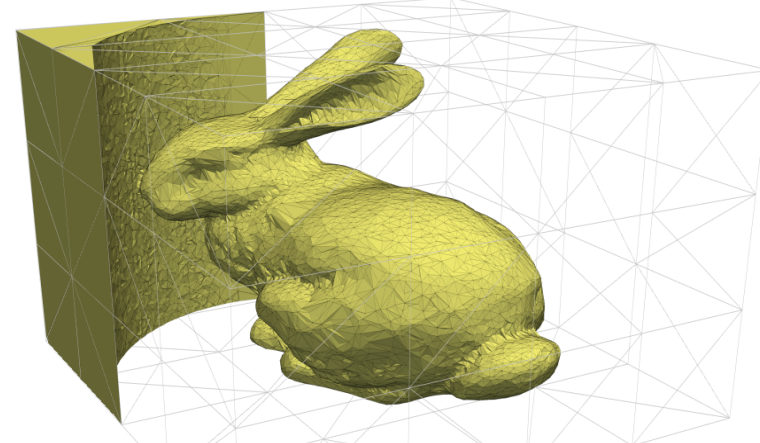

(c) Particionamento ACV + ASmV (2)

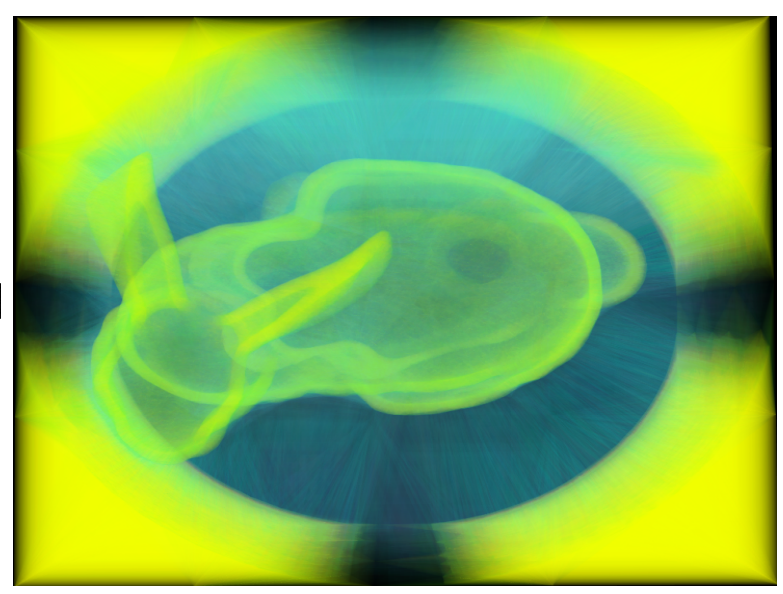

(b) rendering volumétrico da malha

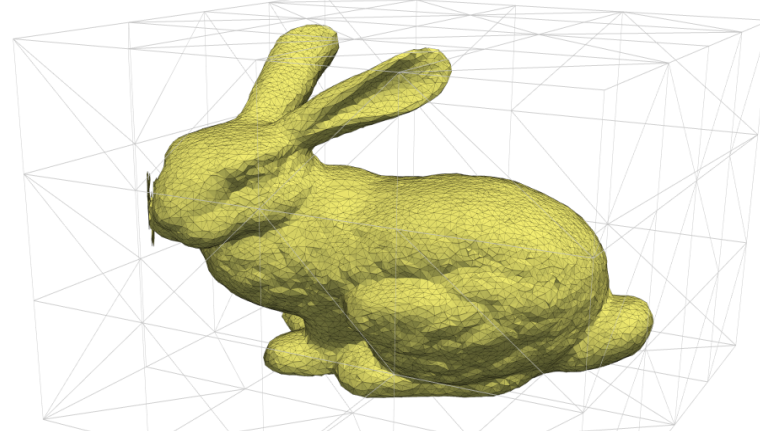

(d) Particionamento ACG (30)+ACV + ASmV

(2)

Figura 5.9: Particionamento tridimensional com critérios geométricos. 


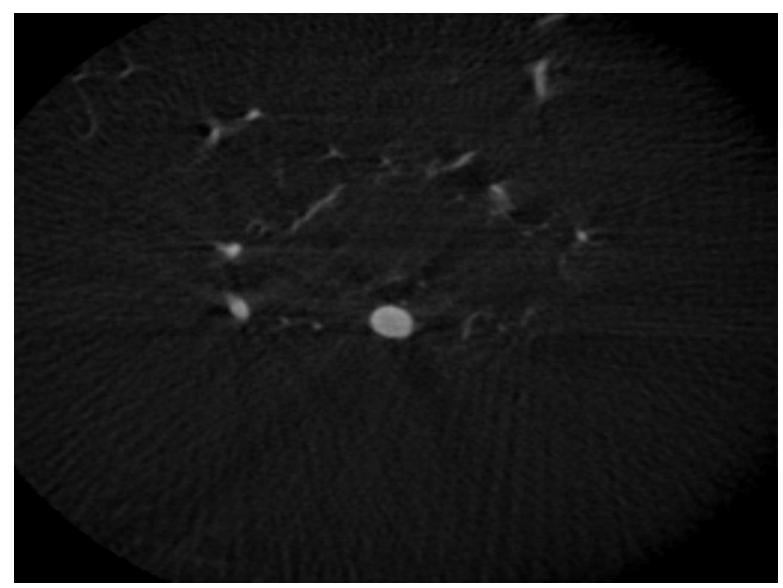

(a) Corte da imagem original

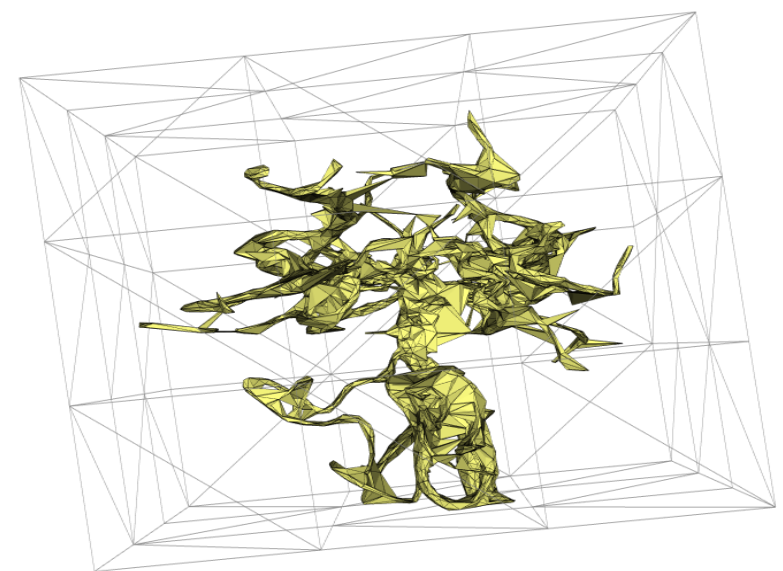

(c) Particionamento $\mathrm{ACV}+\mathrm{ASmV}$ $(60)+\operatorname{ASmS}(2)$

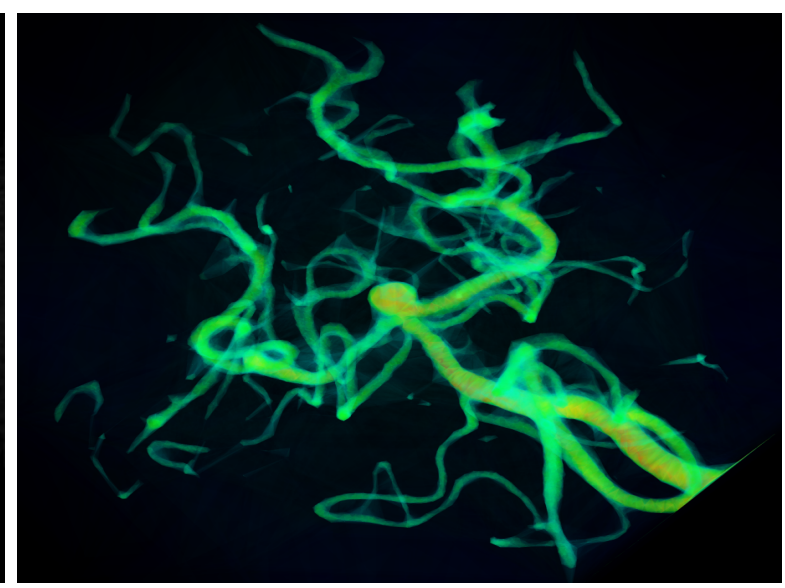

(b) rendering volumétrico da malha

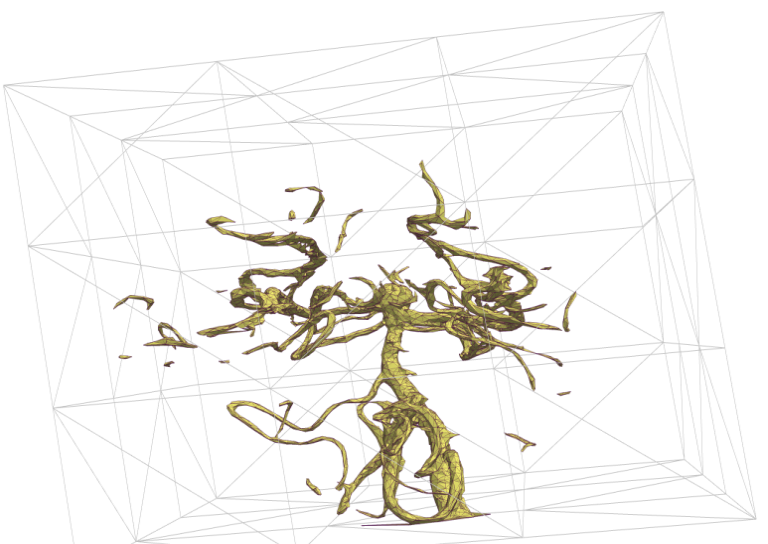

(d) Particionamento $\mathrm{ACV}_{B R}+\mathrm{ASmV}$ $(60)+\operatorname{ASmS}(2)$

Figura 5.10: Exemplo de utilização de operadores com barreira de restrição. 


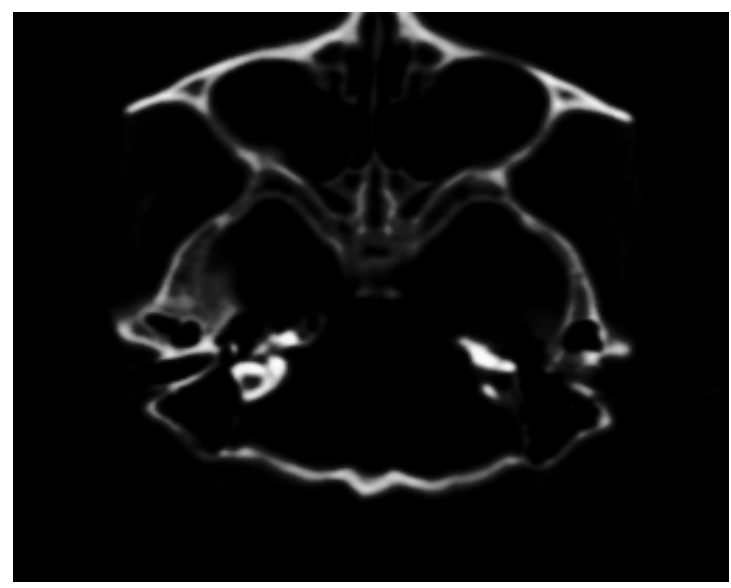

(a) Corte da imagem original

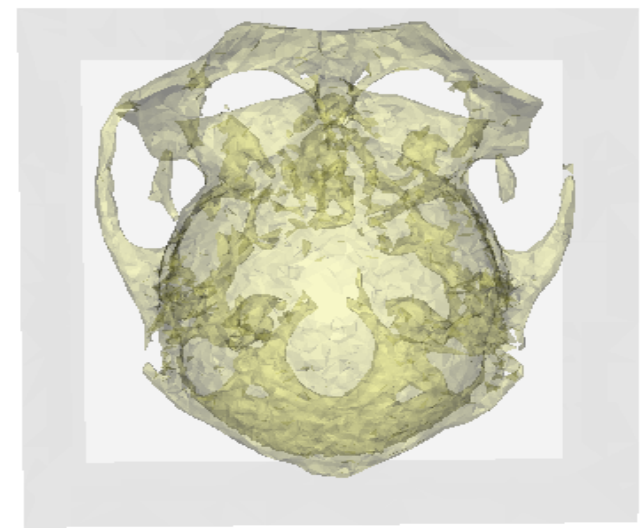

(c) Particionamento $\mathrm{ACV}+\mathrm{ASmV}$ $(60)+\operatorname{ASmS}(2)$

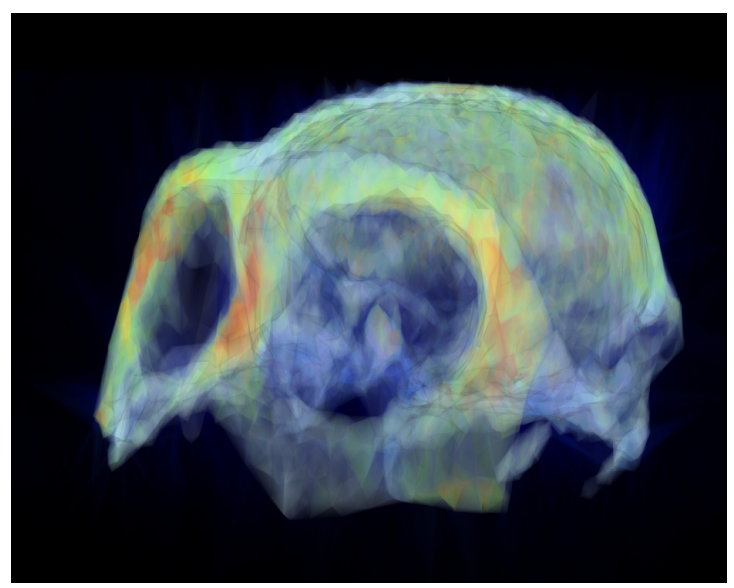

(b) rendering volumétrico da malha

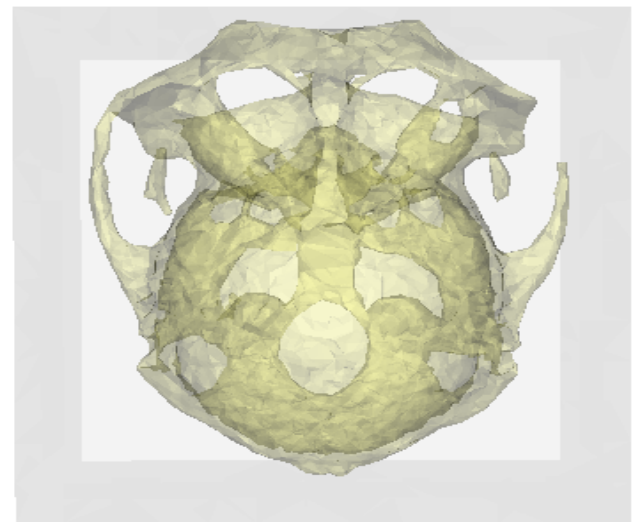

(d) Particionamento $\mathrm{ACV}_{B R}+\mathrm{ASmV}$ $(60)+\operatorname{ASmS}(2)$

Figura 5.11: Exemplo de tratamento uso pela abordagem PCAM. 

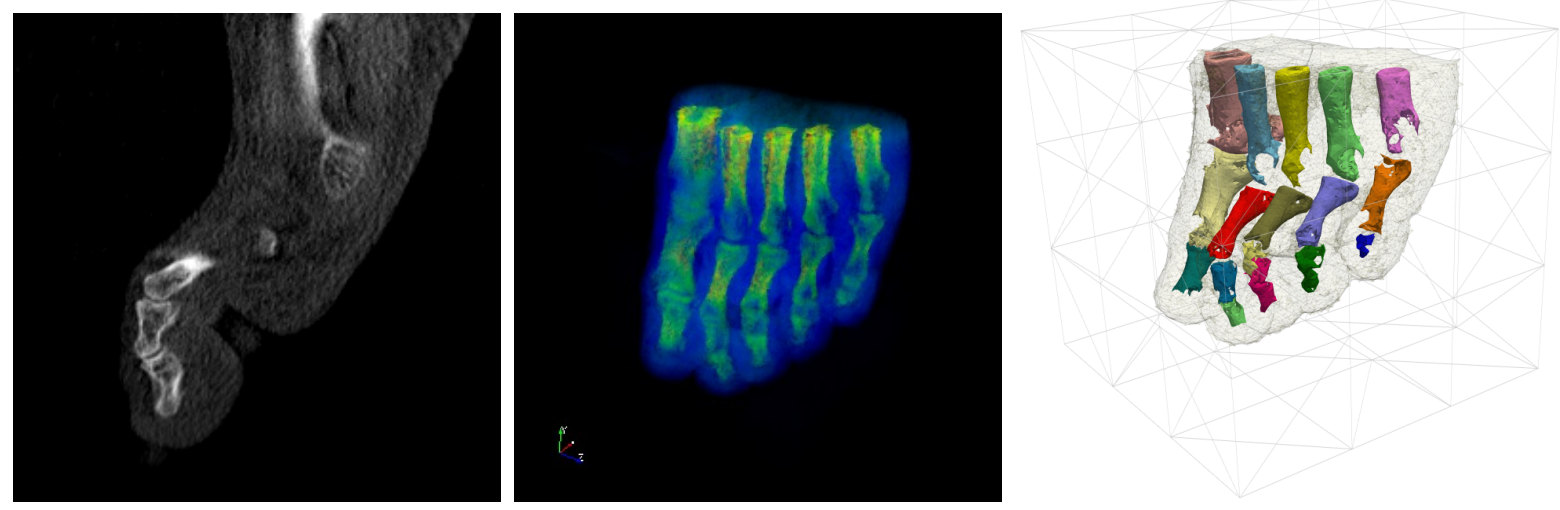

(a) Corte da imagem original

(b) rendering volumétrico da malha

(c) Particionamento $\mathrm{ACV}_{B R}$ $+\mathrm{ASmV}$ (19)
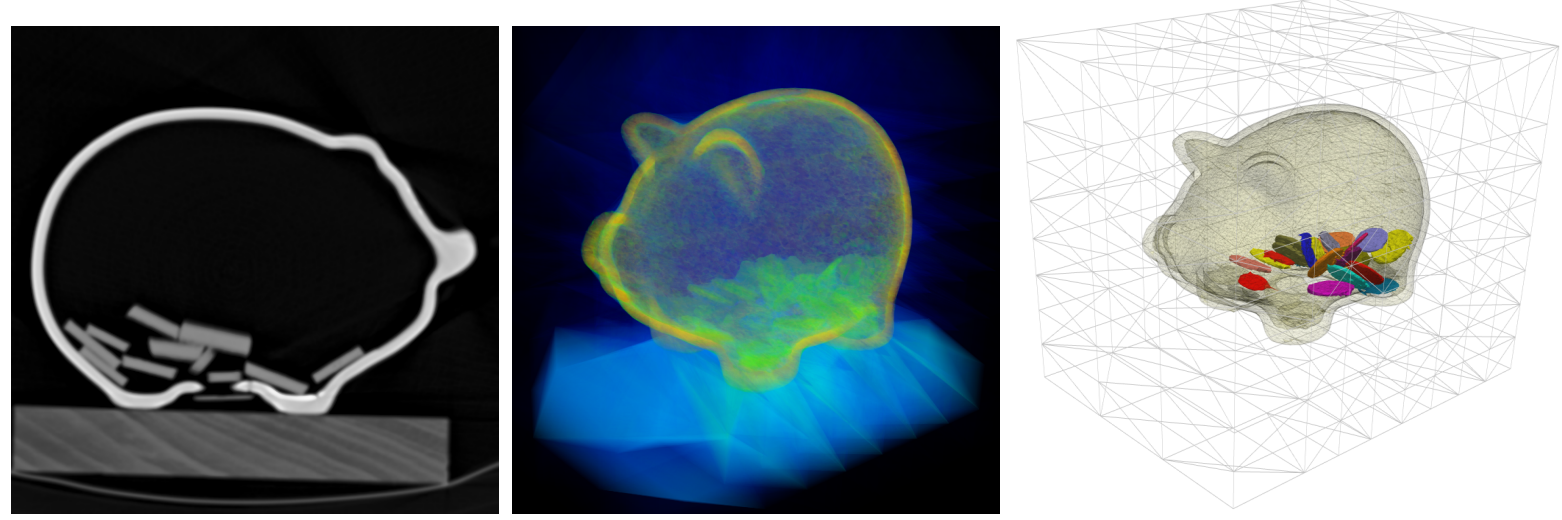

(d) Corte da imagem original

(e) rendering volumétrico da malha (f) Particionamento $\mathrm{ACV}_{B R}$ $+\mathrm{ASmV}(19)$

Figura 5.12: Exemplos de resultados com um número alto de sub-malhas, obtido 


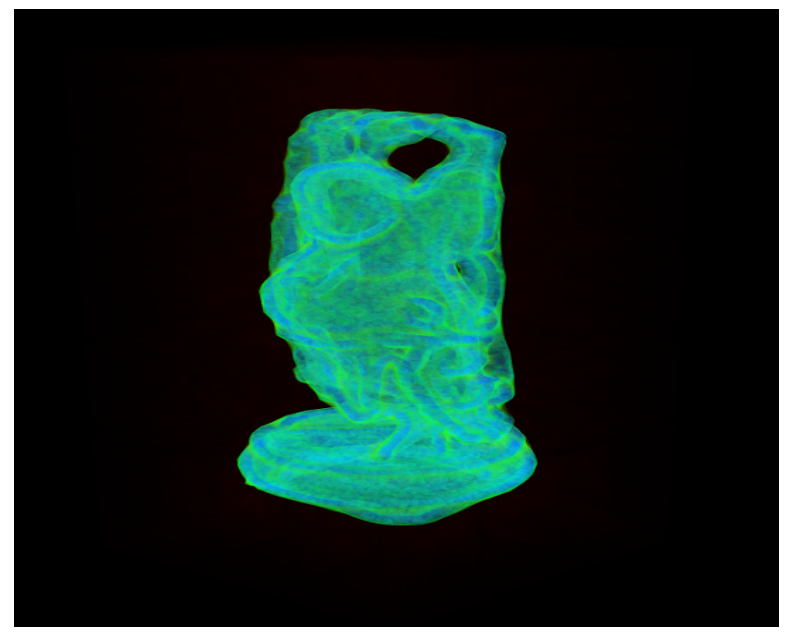

(a) rendering volumétrico da malha

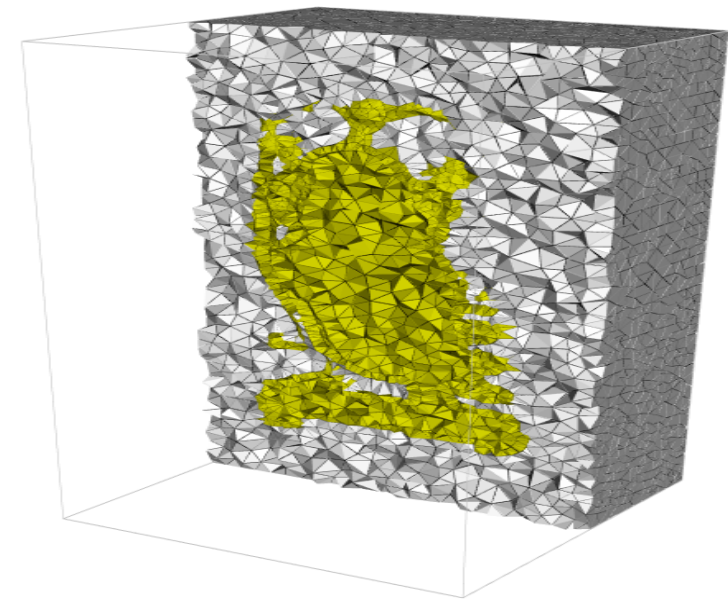

(c) Detalhe de refinamento

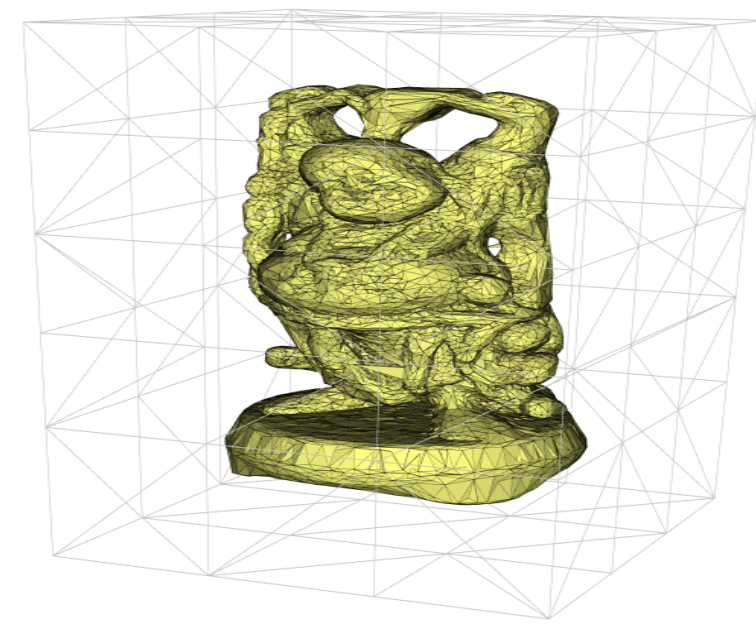

(b) $\mathrm{ACV}_{B R}+\mathrm{ASmV}(2)$

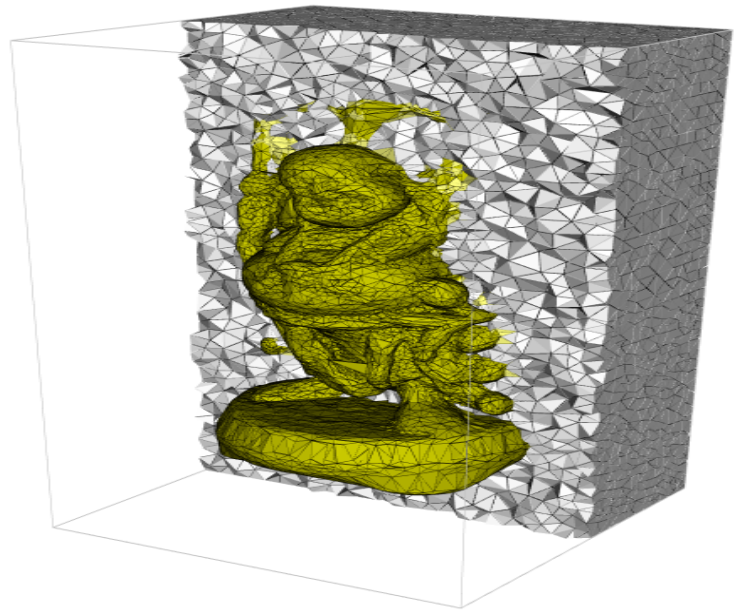

(d) Detalhe de refinamento

Figura 5.13: Exemplo 1 de refinamento tridimensional. Foram utilizados $\mathscr{E}_{v}, B=2$ e $\Xi=5$. 


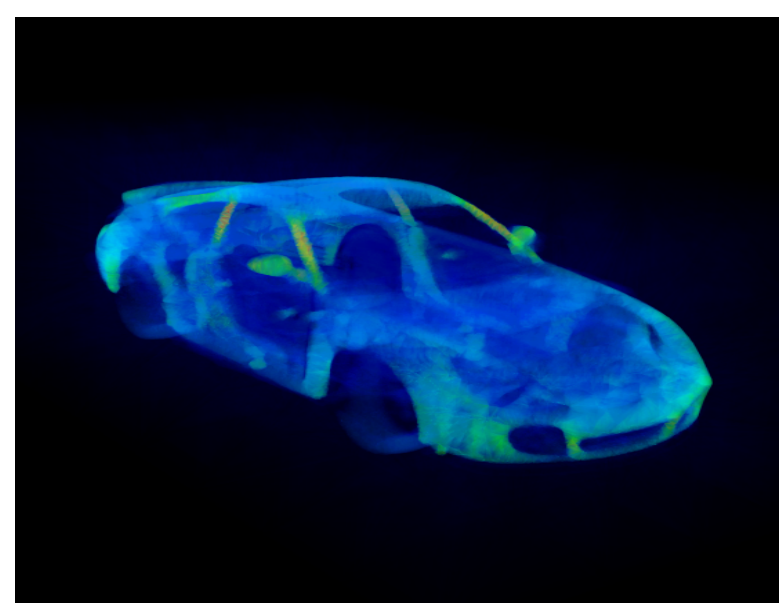

(a) rendering volumétrico da malha

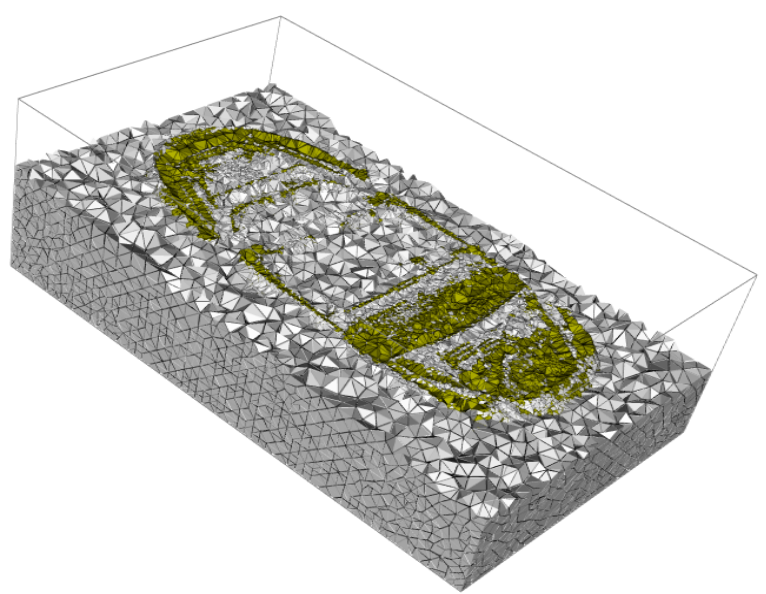

(c) Detalhe de refinamento

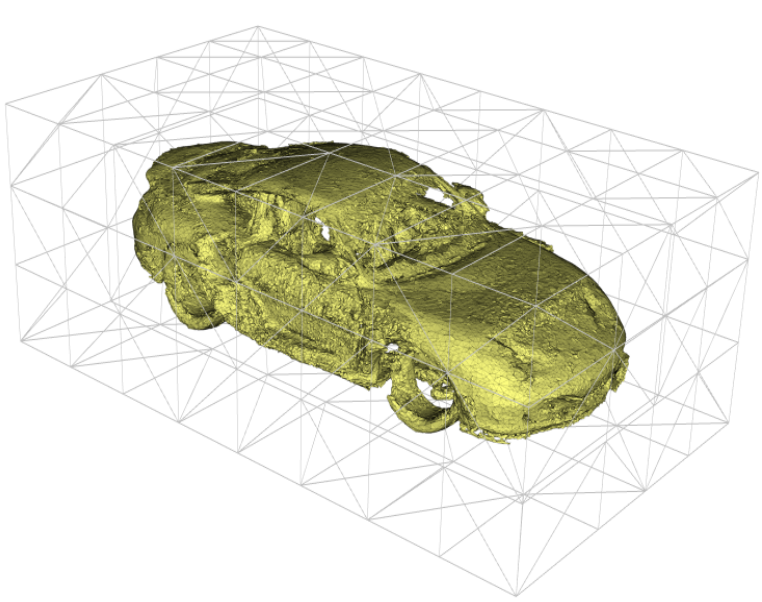

(b) $\mathrm{ACV}_{B R}+\mathrm{ASmV}(2)$

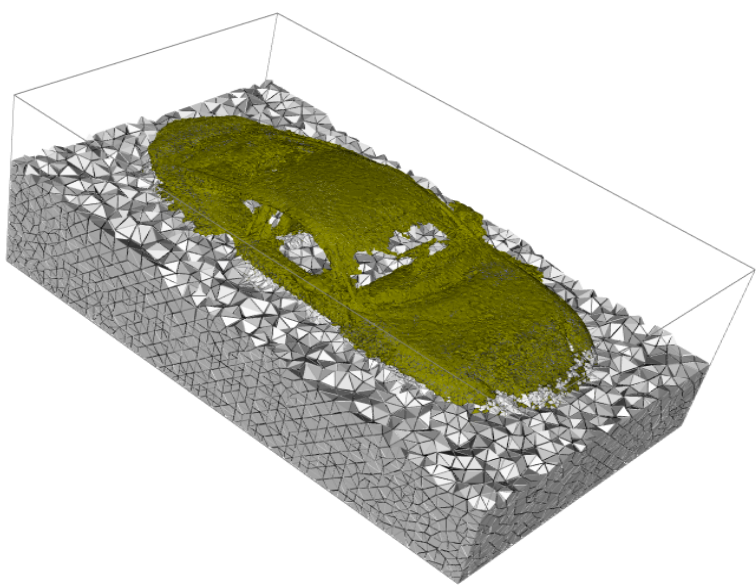

(d) Detalhe de refinamento

Figura 5.14: Exemplo 2 de refinamento tridimensional. Foram utilizados $\mathscr{E}_{v}, B=2$ e $\Xi=8$. 


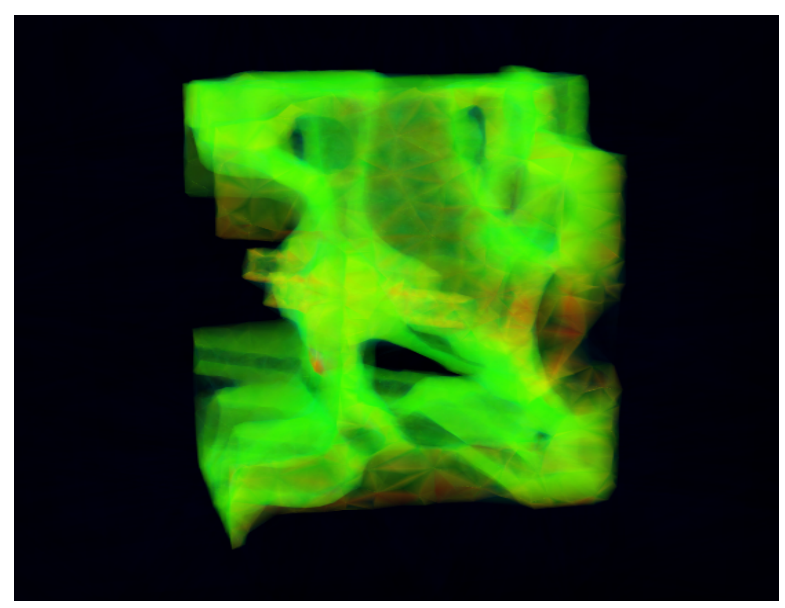

(a) rendering volumétrico da malha

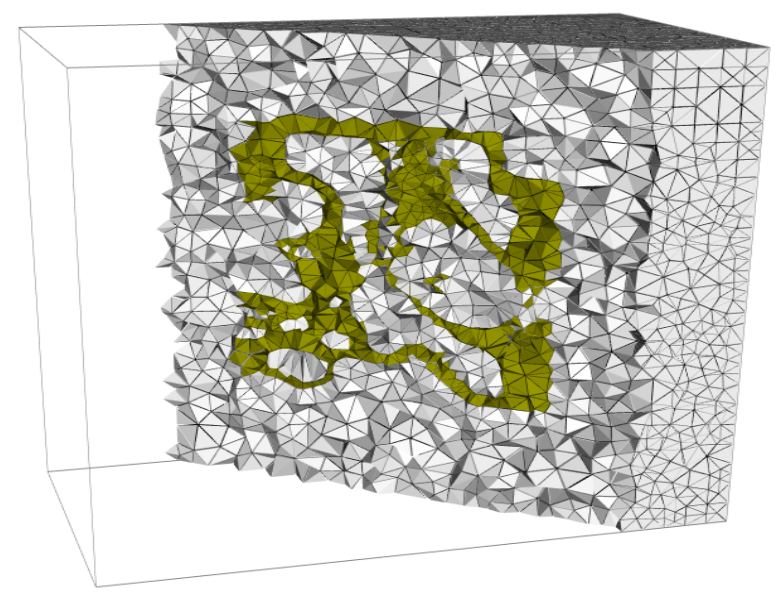

(c) Detalhe de refinamento

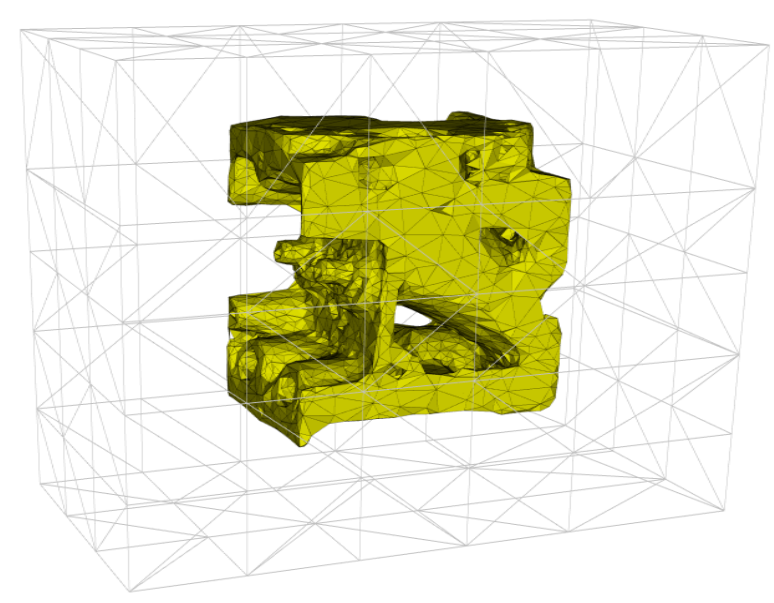

(b) $\mathrm{ACV}_{B R}+\mathrm{ASmV}(2)$

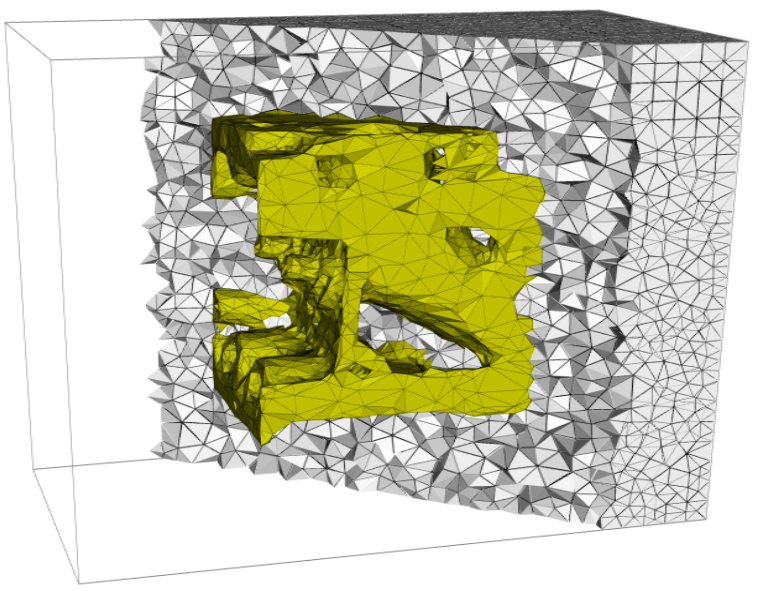

(d) Detalhe de refinamento

Figura 5.15: Exemplo 3 de refinamento tridimensional. Foram utilizados $\mathscr{E}_{v}, B=2$ e $\Xi=6$. 


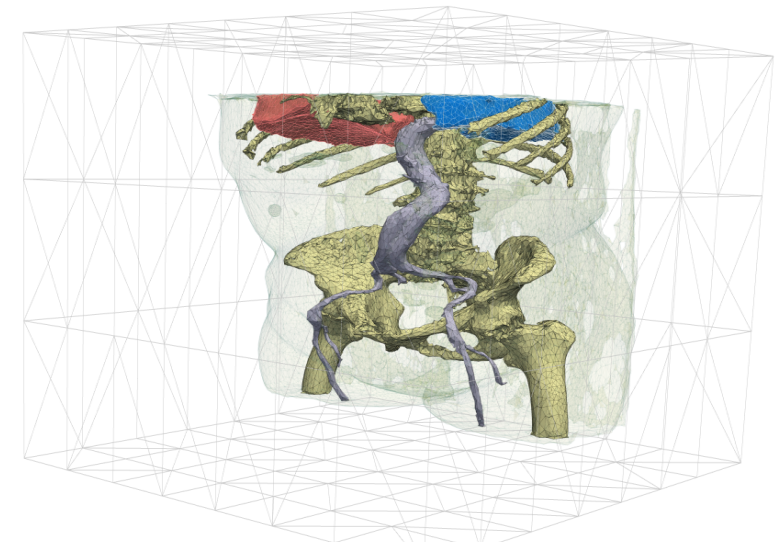

(a) Stent, $\mathscr{E}_{v}+\mathrm{ACV}_{B R}+\mathrm{ASmV}(6)$

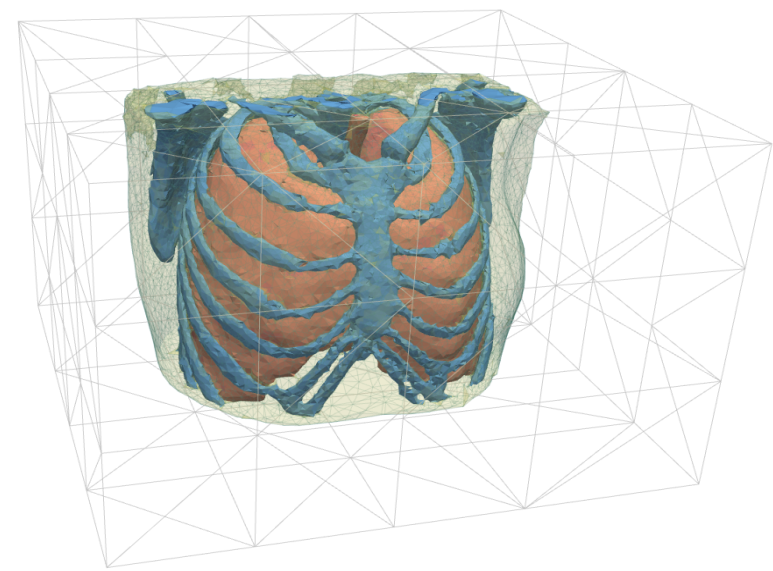

(c) Tórax, $\mathscr{E}_{v}+\mathrm{ACV}_{B R}+\mathrm{ASmV}(4)$

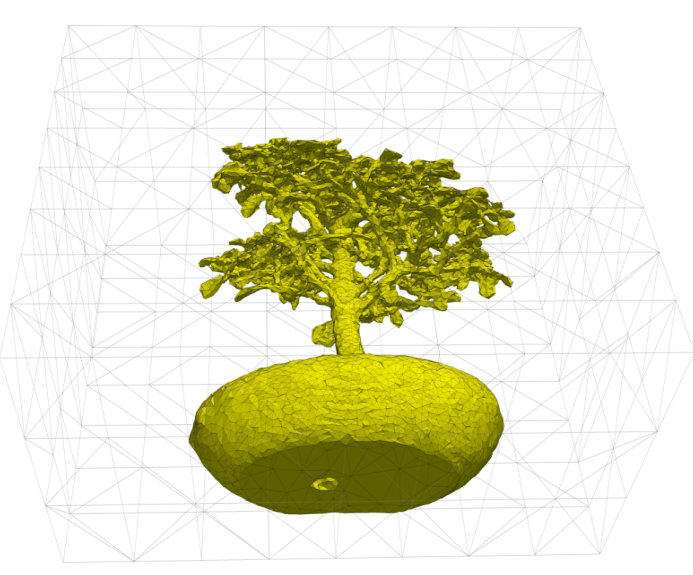

(e) Bonsai $\mathscr{E}_{v}+\mathrm{ACV}_{B R}+\mathrm{ASmV}(2)$

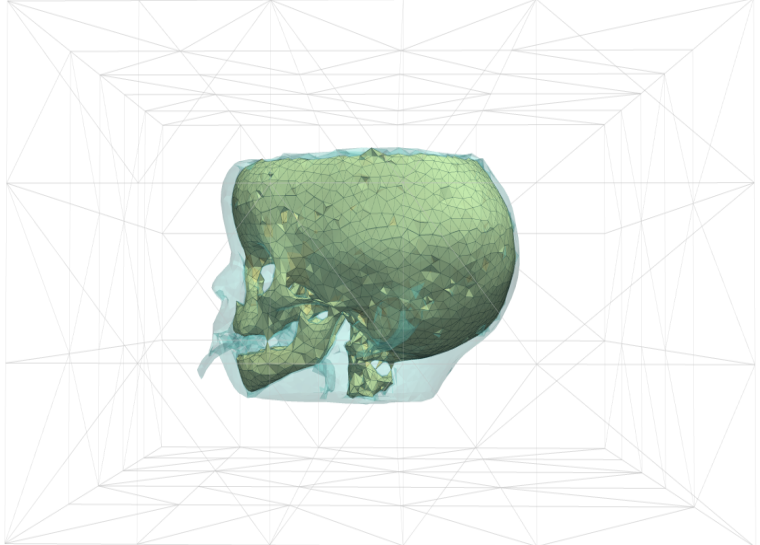

(b) Cabeça de criança , $\mathscr{E}_{v}+\mathrm{ACV}_{B R}+\mathrm{ASmV}$ (3)

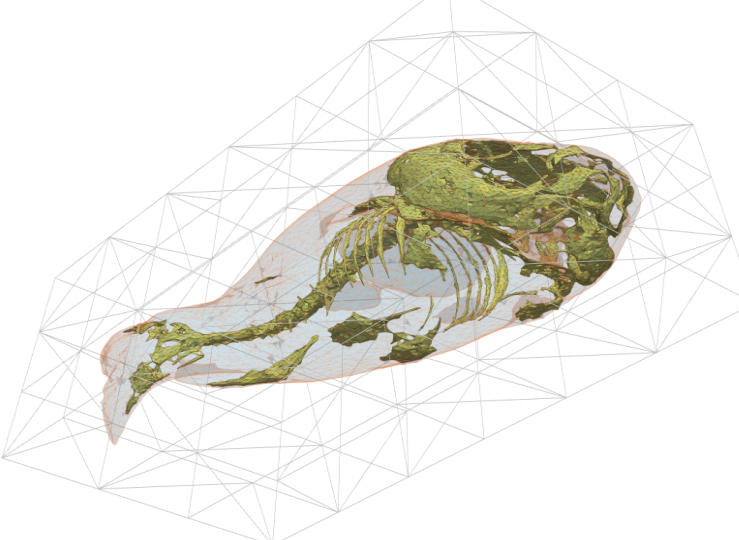

(d) Carpa, $\mathscr{E}_{v}+\mathrm{ACV}_{B R}+\mathrm{ASmV}(60)+\mathrm{ASmS}$ (3)

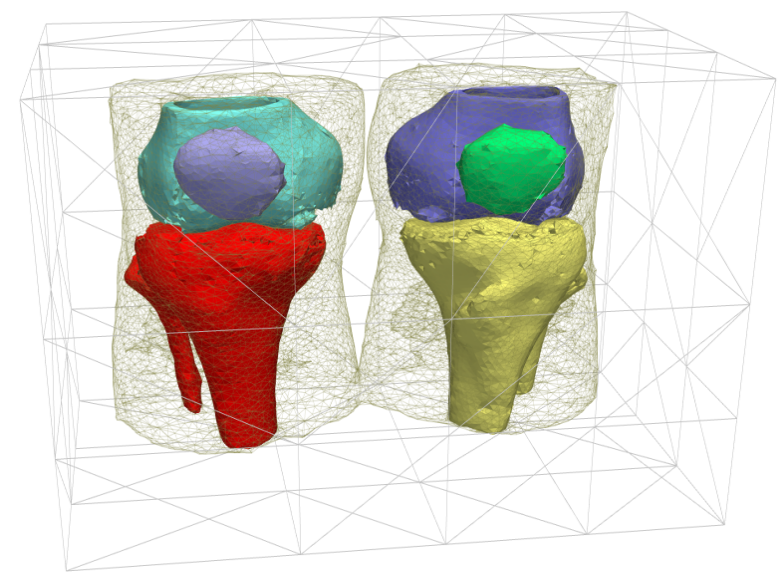

(f) Joelhos $\mathscr{E}_{v}+\mathrm{ACV}_{B R}+\mathrm{ASmV}(8)$

Figura 5.16: Outros particionamentos tridimensionais obtidos 


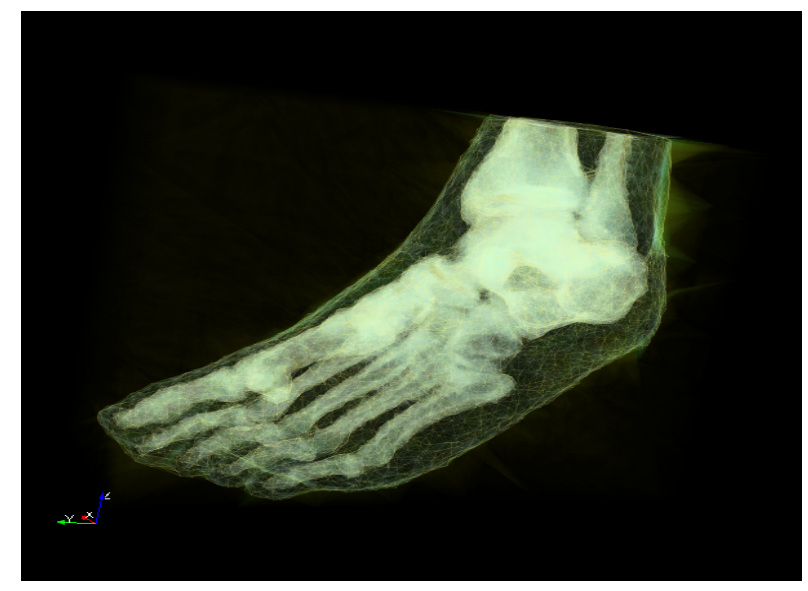

(a) Pé, $\mathscr{E}_{v}$

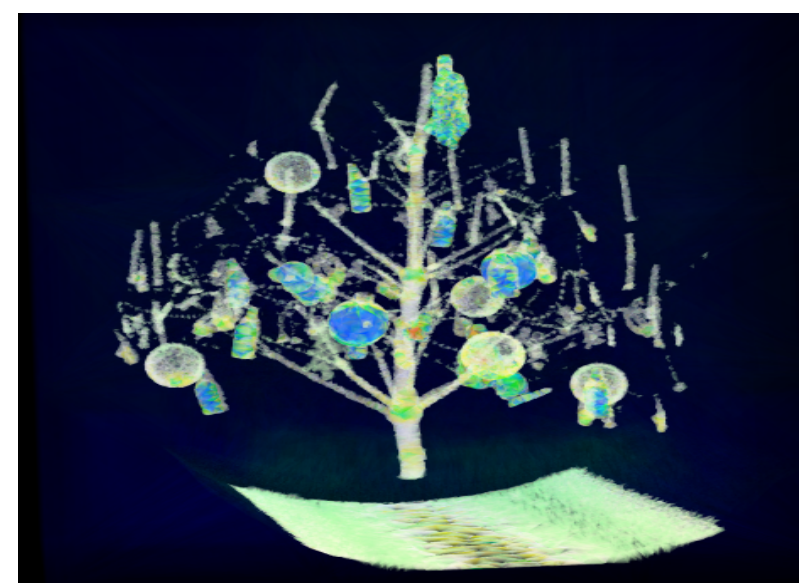

(c) Árvore de natal, $\mathscr{E}_{i}$

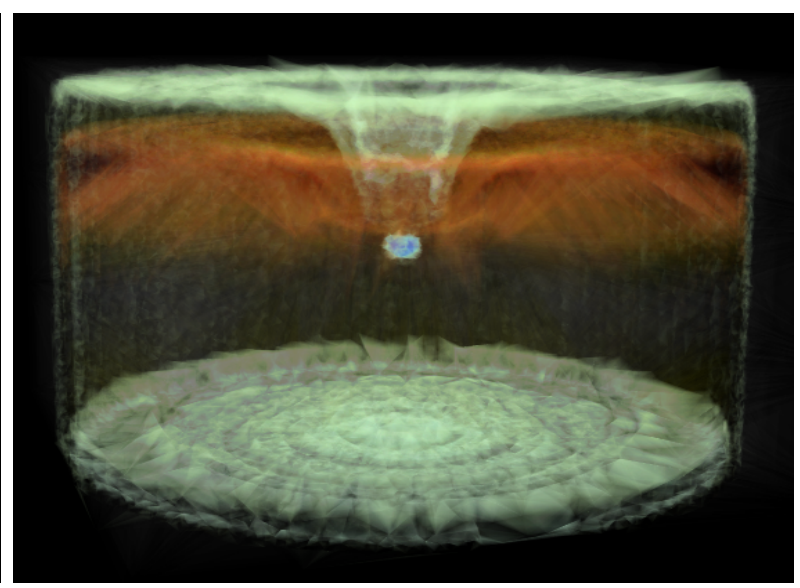

(b) Simulação de fluidos, $\mathscr{E}_{g}$

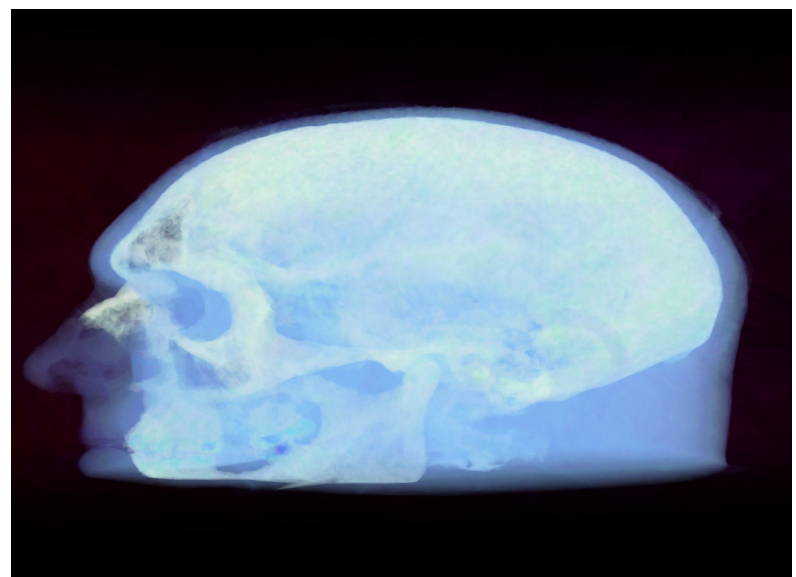

(d) Cabeza (Visible human), $\mathscr{E}_{v}$

Figura 5.17: Outras visualizações de malhas via rendering volumétrico realizadas. 


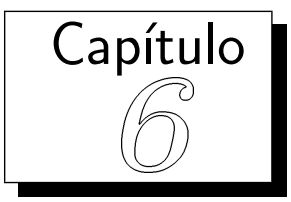

\section{Conclusões e trabalhos futuros}

Apresentamos neste trabalho uma nova técnica, chamada Imesh, relacionada ao contexto de geração de malhas a partir de imagens. Foram desenvolvidas, duas versões do algoritmo, Imesh-2 para o caso bidimensional e Imesh-3 para o caso tridimensional.

Uma característica interessante conseguida na criação deste algoritmo, é que a maioria dos conceitos envolvidos podem ser facilmente estendidos de duas para três dimensões. Este fato é muito importante, pois abre a possibilidade de testar e desenvolver novas idéias no contexto simplificado do algoritmo bidimensional Imesh-2 para logo, se sucedidas, ser estendidas de forma natural para o contexto do algoritmo tridimensional Imesh-3. De fato, nas implementações realizadas, existe uma única versão de muitas operações não dependentes da dimensão, e são utilizadas pelos dois algoritmos.

A técnica proposta introduz uma nova forma, de abordar o problema de geração de malhas a partir de imagens. Diferentemente das abordagens descritas na literatura, a técnica Imesh assume como entrada uma imagem não pré-processada e procura gerar uma malha segmentada, considerando ainda critérios de qualidade nos elementos da malha. Para esse fim, o Imesh divide o problema em três etapas principais Construção de malha, Particionamento de malha e Refinamento de qualidade de malha, como descrito no Capítulo 4.

Uma característica do Imesh é a flexibilidade no processo de Construção de malha, pois permite que diferentes métricas de erro (funções de erro pontuais) sejam empregadas, o que possibilita adequar a triangulação produzida às características de uma determinada 
aplicação. Além disso, as métricas empregadas no processo não precisam percorrer todo o interior de uma célula , para realizar o cálculo de erro. O cálculo baseado no caminhamento das medianas de uma célula ${ }_{d}$, torna a estas métricas ligeiras em comparação com outras métricas da literatura, sendo este um fato muito importante especialmente para o caso do algoritmo tridimensional. O estudo de novas medidas de erro aplicados a novos contextos é uma das áreas que pode originar novas pesquiças no desenvolvimento futuro da técnica Imesh.

Outro fato que caracteriza à técnica Imesh é que ferramentas de segmentação são embutidas no processo de geração de malha, tornando-o mais automatizado. O problema tratado nesse processo é semelhante ao problema de segmentação de imagens, porém, a decomposição simplicial apresenta um novo contexto de segmentação. Diferentemente do contexto de segmentação em imagens o novo contexto se apresenta, normalmente, com uma quantidade muito menor de elementos a serem considerados, originando processo de segmentação mais rápidos. Além de padrões de cor, o processo pode considerar também o aspecto geométrico das células ${ }_{d}$ da malha, obtendo dessa forma particionamentos dificilmente alcanzados no contexto de segmentação de imagens, como os resultados mostrados nas Figuras 5.4 e 5.9. Por outro lado, foram apresentados vários operadores de agrupamento (Seção 4.2.2) desenvolvidos para serem combinados e apresentar diferentes alternativas no processo. Novas opções e combinações de particionamento devem também ser pesquisadas em trabalhos futuros. Uma dessas alternativas poderia ser a inclusão da opção de segmentação por campos aleatórios Markovianos (Seção 4.2.3), no contexto dos operadores de agrupamento (Seção 4.2.2).

O desenvolvimento da etapa de Refinamento de qualidade de malha garante critérios nas malhas geradas que direcionam os modelos produzidos pelo Imesh para seu emprego em simulações numéricas, sendo esta uma parte importante da metodologia apresentada. No entanto, o estudo e implementação de algoritmos de refinamento tridimensional, no contexto do Imesh, com uma garantia de qualidade mais profunda, é um tema que poderia também ser estudado no futuro. Pretendemos investigar qual o melhor algoritmo de refinamento 3D para o caso do Imesh, pois a literatura não apresenta uma estratégia capaz de refinar, como qualidade, múltiplas malhas simultaneamente. Tais estudos podem tomar como base os trabalhos de Shewchuk (1997) e de Alliez et al. (2005), os quais apresentam bons resultados, mas sempre refinando apenas um único domínio. Como adaptar tais métodos no contexto 
do Imesh será um desafio importante.

Adicionalmente, antes de efetuar o refinamento para garantir a qualidade dos elementos da malha, pode-se realizar uma simplifição de pontos internos e de fronteira de submalhas. A eliminação de um ponto interior em uma sub-malha pode ser realizada porque esta operação nunca afeta às fronteiras estabelecidas. Por outro lado, a eliminação de pontos sobre a fronteira pode ser executada unicamente no caso de pontos colineares, ou coplanares no caso tridimensional. Antes de realizar esta simplificação deve ser considerado que os pontos eliminados podem afetar informações relevantes para a representação da imagem. Então, na verdade, esta é uma opção que o usuário poderia adotar ou não. Tal processo de simplificação poderia ser baseado nos trabalhos realizados por ALLIEZ et al. (2002) e Vieira et al. (2003).

Estudar o emprego das malhas geradas pelo algoritmo Imesh tanto para fins de simulação numérica quanto para visualização, é outro trabalho que pretendemos desenvolver. Um exemplo disto foi mostrado na Figura 5.17 (b), onde o Imesh foi utilizado para modelar e visualizar um campo de pressões gerado por um software de simulação de fluidos (Castelo et al., 2000). Desta forma o Imesh poderia introduzir uma nova funcionalidade dentro de tal contexto. O emprego do Imesh em problemas de modelagem de estruturas mecânicas também pode ser investigado. Como mostrado nas Figuras 5.15 e 5.14 , o Imesh é capaz de modelar estruturas mecânicas de forma bastante rápida, evitando o exaustivo trabalho de $C A D$. Desta forma, se bem sucedido, se estaria introduzindo uma nova metodologia de modelagem nas áreas de engenharia.

Uma abordagem não diretamente relacionada ao problema de geração de malhas a partir de imagens, mas que possui uma fundamentação teórica relevante para este contexto é o trabalho proposto por Boissonnat e Oudot (2005). Embora trate do problema de geração de superfícies a partir de nuvens de pontos, esse trabalho apresenta uma fundamentação teórica de garantia de reconstrução correta que pode ser adaptada a problemas de geração de malhas a partir de imagens. Propomos também, como trabalho futuro a adaptação das idéias desse trabalho no contexto do algoritmo Imesh.

Como pode ser observado, a partir dos comentários acima, a nova abordagem descrita neste trabalho apresenta resultados bastante promissores com aplicações em diversas áreas, abrindo ao mesmo tempo um horizonte de novos desafios para o problema de geração de malhas a partir de imagens. 


\section{Referências Bibliográficas}

[A. Liu(1994)] A. LiU, B. Relationship between tetrahedron shape measeures. Departament of Computer Science, University of Alberta, Edmonton, AB, Canadá T6G 2H1, v. 34, p. 268-287, 1994.

[Adams e Bischof(1994)] Adams, R.; Bischof, L. Seeded region growing. IEEE Trans. Pattern Anal. Mach. Intell., v. 16, n. 6, p. 641-647, 1994.

[Alliez et al.(2005)] Alliez, P.; Cohen-Steiner, D.; Yvinec, M.; Desbrun, M. Variational tetrahedral meshing. ACM Trans. Graph., v. 24, n. 3, p. 617-625, 2005.

[ALLIEZ et al.(2002)] ALLIEZ, P.; COLIN, O.; M. Isotropic surface remeshing. 2002. Disponível em citeseer.ist.psu.edu/alliez03isotropic.html

[Amenta et al.(2001)] Amenta, N.; Choi, S.; Kolluri, R. The power crust, unions of balls, and the medial axis transform. Computational Geometry: Theory and Applications, v. 19, n. 2-3, p. 127-153, 2001.

[Auer et al.(1999)] Auer, L.; Radetzky, A.; Et Al., C. W. Visualization for planning and simulation of minimally invasive neurosurgical procedures. Medical Image Computing and Computer-Assisted Intervention - MICCAI'99, Lecture Notes in Computer Science, v. 1679, p. 1199-1209, 1999.

[Aurenhammer.(1991)] Aurenhammer., F. Voronoi diagrams - a survey of a fundamental geometric data structure. In: ACM Computing Surveys, 1991, p. 345-405.

[B. S.Baker(1988)] B. S.BAkER, E. G. C. R. Nonobtuse triangulation of polygons. Discrete and Computational Geometry, 1988. 
[Bajaj et al.(1996)] BAJAJ, C.; Coyle, E.; Lin., K. Arbitrary topology shape reconstruction from planar cross sections. In: Graphical Models and Image Processing, 1996, p. $524-543$.

[Bern e Eppstein(1992)] Bern, M.; Eppstein, D. Mesh generation and optimal triangulation. World Cientific Publishing Co., p. 23-90, 1992.

[Berti(2004)] BERTI, G. Image-based unstructured 3d mesh generation for medical applications. In: ECCOMAS - European Congress On Computational Methods in Applied Sciences and Engeneering, 2004.

[Bertin et al.(1994)] Bertin, E.; Marchand-Maillet, S.; Chassery, J. Optimization in voronoi diagrams. In: SerRA, J.; Sollle, P., eds. Mathematical Morphology and Its Applications to Image Processing, Kluwer Academic, p. 209-216, 1994.

[Bielser e Gross.(2000)] Bielser, D.; Gross., M. Interactive simulation of surgical cuts. Proceedings of Pacific Graphics, IEEE Computer Society Press, p. 116-125, 2000.

[Boissonnat(1988)] Boissonnat, J.-D. Shape reconstruction from planar cross sections. In: Computer Vision, Graphics and Image Processing, 1988, p. 1-29.

[Boissonnat et al.(2002)] Boissonnat, J.-D.; Devillers, O.; Pion, S.; Teillaud, M.; Yvinec, M. Triangulations in cgal. Computational Geometry - Theory and Applications, v. 22, p. 5-19, 2002.

[Boissonnat e Oudot(2005)] Boissonnat, J.-D.; Oudot, S. Provably good sampling and meshing of surfaces. Graph. Models, v. 67, n. 5, p. 405-451, 2005.

[Bros(2006)] Bros, W. Demon of tazmania. 2006.

Disponível em http://http://www.warnerbros.com./

[Carr et al.(2006)] Carr, H.; Moller, T.; Snoeyink, J. Artifacts caused by simplicial subdivision. IEEE TVCG, v. 12, n. 2, p. 231-242, 2006.

[Castelo et al.(2000)] Castelo, A.; Tomé, M.; Cesar, M.; Cuminato, J.; Mckee, S. Freeflow: An intregated simulation system for three-dimensional free surface flows. Journal of Computing and Visualization in Science, v. 2, p. 199-210, 2000. 
[Cebral e Lohner(1999a)] Cebral, J.; Lohner, R. From medical images to cfd meshes. 1999a.

Disponível em citeseer.nj.nec.com/cebralandr99from.html

[Cebral e Lohner(1999b)] Cebral, J.; Lohner, R. From medical images to cfd meshes. In: Proceedings of the 8th International Meshing Roundtable, 1999b, p. 321-331.

[Cheng et al.(2000)] Cheng, S.; Dey, T.; Edelsbrunner, H. Sliver exudation. In: journal of the ACM, 2000, p. 883-904.

[Cheng e Dey(1999)] Cheng, S.-W.; Dey, T. Improved constructions of delaunay-based contour surfaces. In: ACM Symposium on Solid Modeling and Applications, 1999, p. 322-323.

[Cheng e Dey(2002)] Cheng, S. W.; Dey, T. K. Quality meshing with weighted delaunay refinement. In: Proceedings of the thirteenth annual ACM-SIAM symposium on Discrete algorithms, Society for Industrial and Applied Mathematics, 2002, p. 137-146.

Disponível em citeseer.nj.nec.com/473003.html

[Cheng et al.(2004)] Cheng, S.-W.; Dey, T. K.; Ramos, E. A.; RaY, T. Quality meshing for polyhedra with small angles. In: SCG '04: Proceedings of the twentieth annual symposium on Computational geometry, New York, NY, USA: ACM Press, 2004, p. $290-299$.

[Chew(1993)] CHew, L. P. Garanteed-quality mesh generation for curved surfaces. In: ACM Symposium on Computational Geometry, 1993, p. 274-280.

[Chew(1997)] Chew, L. P. Guaranteed-quality delaunay meshing in 3d (short version). In: SCG '97: Proceedings of the thirteenth annual symposium on Computational geometry, New York, NY, USA: ACM Press, 1997, p. 391-393.

[Chew(1989)] CHEw, P. Guaranteed-quality triangular meshes. Relatório Técnico, Departament of Computer Science, Cornell University, 1989.

[Ciampalini et al.(1997)] Ciampalini, A.; Cignoni, P.; Montani, C.; Scopigno, R. Multiresolution decimation based on global error. The Visual Computer, v. 13, n. 5, p. 228-246, 1997.

Disponível em citeseer.ist.psu.edu/ciampalini97multiresolution.html 
[Cohen-Steiner et al.(2004)] Cohen-Steiner, D.; Alliez, P.; Desbrun, M. Variational shape approximation. ACM Trans. Graph., v. 23, n. 3, p. 905-914, 2004.

[Coleman e Scotney(2005)] Coleman, S.; Scotney, B. Mesh modelling for sparse image data sets. IEEE International Conference on Image Processing, v. 2, p. II- 1342-5, 2005.

[Comer e Delp(2000)] Comer, M. L.; Delp, E. J. The em/mpm algorithm for segmentation of textured images: Analysis and further experimental results. IEEE Transactions on Image Processing, v. 9, n. 10, 2000.

Disponível em citeseer.ist.psu.edu/106627.html

[Cuadros-Vargas e Nonato(2004)] Cuadros-Vargas, A. J.; Nonato, L. G. Bidimensional delaunay mesh generation from images. In: XXV CILAMCE, Iberian Latin American Congress on Computational Methods, 2004.

[Cuadros-Vargas e Nonato(2006)] Cuadros-Vargas, A. J.; Nonato, L. G. Imesh: Quality mesh generation from images. ECCOMAS - European Congress On Computational Methods in Applied Sciences and Engeneering, 2006.

[Cuadros-Vargas et al.(2006a)] Cuadros-Vargas, A. J.; Nonato, L. G.; Batista, L. C. G. J. A bidimensional stochastic mehotd for segment simplicial meshes generated from images. Brazilian Symposium on Computer Graphics and Image Processing (SIBGRAPI), 2006a.

[Cuadros-Vargas et al.(2005)] Cuadros-Vargas, A. J.; Nonato, L. G.; Minghim, R.; Etiene, T. Imesh: An image based quality mesh generation technique. In: Brazilian Symposium on Computer Graphics and Image Processing, 18 (SIBGRAPI), IEEE Computer Society, 2005.

[Cuadros-Vargas et al.(2006b)] Cuadros-Vargas, A. J.; Nonato, L. G.; Tejada, E.; ERTL, T. Generating segmented tetrahedral meshes from regular volume data for simulation and visualization applications. In: Computational Modelling of Objects Represented in Images: Fundamentals, Methods and Applications (CompIMAGE), 2006b.

[Dari et al.(2000)] Dari, E.; Cantero, M.; Feijóo, R. Computational arterial flow modeling using a parallel navier-stokes solver. ECCOMAS 2000, 2000. 
[Earth(2006)] EARTH, G. Google earth - a 3d interface of the planet. 2006.

Disponível em http://earth.google.com/

[Edelsbrunner(2000)] EDELsBrunner, H. Triangulations and meshes in computational geometry. In: Acta Numerica, 2000, p. 133-213.

[Edelsbrunner(2001)] Edelsbrunner, H. Geometry and topology for mesh generation. Cambridge Monographs on Applied and Computational Mathematics. Cambridge, 2001.

[Edelsbrunner e Guoy(2001)] Edelsbrunner, H.; Guoy, D. An experimental study of sliver exudation. 2001.

Disponível em citeseer.ist.psu.edu/edelsbrunner01experimental.html

[Edelsbrunner et al.(2000)] Edelsbrunner, H.; Li, X.-Y.; Miller, G.; Stathopoulos, A.; Talmor, D.; Teng, S.-H.; Üngör, A.; Walkington, N. Smoothing and cleaning up slivers. 2000, p. 273-277.

Disponível em citeseer.nj.nec.com/edelsbrunner00smoothing.html

[EMuseum(2006)] EMuseum, M. Minnesota state university, mankato - south american places. 2006.

Disponível em http://www.mnsu.edu/emuseum/prehistory/latinamerica/south/ sites

[Erlangen(2005)] ERLANGEN Real world medical datasets - university of tübingen. 2005. Disponível em http://www.gris.uni-tuebingen.de/areas/scivis/volren/index. html

[Fabri(2001)] FABRI, A. Cgal - the computational geometry algorithm library. 10th International Meshing Roundtable, p. 137-142, 2001.

Disponível em http://www.imr.sandia.gov/papers/imr10/fabri01.pdf.gz

[Fabri et al.(2000)] Fabri, A.; Giezeman, G.; Kettner, L.; Schirra, S.; Schonherr, S. The design of cgal a computational geometry algorithms library. 2000.

Disponível em citeseer.ifi.unizh.ch/article/fabri98design.html

[Ferrant et al.(2001)] Ferrant, M.; MacQ, B.; NABAvi, A.; ET Al. Deformable modeling for characterizing biomedical shape changes. LECT NOTES COMPUT SC, v. 1953, p. $235-248,2001$. 
[Fortune(1992)] Fortune, S. Voronoi diagrams and delaunay triangulation. In: F.K., H.; D.Z., D., eds. Computing in Euclidean Geometry, v. 1 de Lecture Notes Series on Computing, Singapore: World Scientific, p. 193-233, 1992.

[Fortune(1994)] Fortune, S. Voronoi diagrams and delaunay triangulation. In: Computing in Euclidean Geometry, Wor1d Scientific Pub. Co., 1994, p. 193-233.

[García et al.(1999)] García, M.; SAPpA, A.; Vintimilla, B. Efficient approximation of gray-scale images through bounded error triangular meshes. In: IEEE Intern. Conf. on Image Processing, 1999, p. 168-170.

[Garland e Heckbert(1995)] Garland, M.; HeCKBert, P. Fast polygonal approximation of terrains and height fields. Relatório Técnico CMU-CS-95-181, Carnegie Mellon University, 1995.

[Geiger(1993a)] GEIGER, B. Three dimensional modeling of human organs and its application to diagnosis and surgical planning. Relatório Técnico 2105, INRIA, 1993a.

[Geiger(1993b)] GEIGER, B. Three dimensional modeling of human organs and its application to diagnosis and surgical planning. Relatório Técnico, INRIA (Sophia-Antipolis), France, 1993b.

[Geographic(2006)] Geographic, N. Animal world. 2006.

Disponível em http://www.nationalgeographic.com/animals./

[German e German(1984)] German, S.; German, D. Stochastic relaxation, gibbs distributions, and the bayesian restoration of images. IEEE PAMI, v. 6, p. 721-741, 1984.

[Gevers e Smeulders(1997)] Gevers, T.; Smeulders, A. Combining region splitting and edge detection through guided delaunay image subdivision. 1997.

Disponível em citeseer.ist.psu.edu/gevers97combining.html

[Grosso et al.(1997)] Grosso, R.; Lürig, C.; ErTL, T. The Multilevel Finite Element Method for Adaptive Mesh Optimization and Visualization of Volume Data. In: YageL, R.; Hagen, H., eds. Visualization '97\%, IEEE Computer Society Press, 1997.

[Guibas et al.(1992)] Guibas, L.; Knuth, D.; Sharir, M. Randomized incremental construction of delaunay and voronoi diagrams. Algorithmica, v. 7, p. 381-413, 1992. 
[Guillaume et al.(2004)] Gulllaume, L.; Florent, D.; Atilla, B. Curvature tensor based triangle mesh segmentation with boundary rectification. In: Computer Graphics International, 2004, p. 10-17.

[Guthe et al.(2002)] Guthe, S.; Wand, M.; Gonser, J.; ER, W. S. Interactive rendering of large volume data sets. In: VIS '02: Proceedings of the conference on Visualization '02, 2002, p. 53-60.

[Hale(2001a)] Hale, D. Atomic images - a method for meshing digital images. In: Proceedings, 10th International Meshing Roundtable, Sandia National Laboratories, 2001a, p. $185-196$.

Disponível em citeseer.nj.nec.com/478480.html

[Hale(2001b)] HaLe, D. Atomic Images - a method for meshing digital images. In: 10th International Meshing Roundtable, 2001b, p. 185-196.

[Haralick e Shapiro(1992)] Haralick, R.; Shapiro, L. Computer and robot vision, v. 1. Addison-Wesley Publishing Company, 1992.

[Hojjatoleslami e Kittler(1998)] HojJatoleslami, S. A.; Kittler, J. Region growing: A new approach. IEEE Transactions on Image Processing, Vol. 7, NO. 7, July 1998, v. 7, n. 7, p. 1979-1984, 1998.

[Huang e Hsu(1994)] HuAng, C.-L.; Hsu, C.-Y. A new motion compensation method for image sequence coding using hierarchical grid interpolation. IEEE Transactions on Circuits and Systems for Video Technology, v. 4, p. 44-51, 1994.

[Jain(1989)] Jain, A. Fundamentals of digital image processing. Prentice Hall, 1989.

[Kaufman(1998)] KaUfman, A. Advances in volume visualization. In: SIGGRAPH'98, Course Notes no. 24, 1998.

[Keener e Panfilov.(1995)] Keener, J.; Panfilov., A. Three dimensional propagation in the heart: The effects of geometry and fiber orientation on propagation in myocardium. Cardiac Electrophysiology, p. 335-348, 1995.

[Knupp(2001)] Knupp, P. Algebraic mesh quality metrics. Society for Industrial and Applied Mathematics, SIAM J, Sci. Comput., v. 23, n. 5, p. 193-218, 2001. 
[Kocharoen et al.(2005)] Kocharoen, P.; Ahmed, K.; Rajatheva, R.; Fernando, W. Adaptive mesh generation for mesh-based image coding using node elimination approach. IEEE International Conference on Communications, v. 3, p. 2052-2056, 2005.

Disponível em http://ieeexplore.ieee.org/xpls/abs_all.j.sp?arnumber=1494699

[Li(2000)] LI, X.-Y. Spacing control and sliver-free delaunay mesh. In: Proceedings, 9th International Meshing Roundtable, 2000, p. 295-306.

Disponível em http://www.imr.sandia.gov/papers/imr9/li00.ps.gz

[Lo.(1997)] Lo., S. Optimization of tetrahedral meshes baded on element shape measures. Departament of Civil and Structural Sngeneering, The University of Hong Kong, Hong Kong, Computers and Computers, v. 63, n. 5, p. 951-961, 1997.

[Marroquin et al.(1987)] Marroquin, J.; Mitter, S.; Poggio, T. Probabilistic solution fo ill-posed problems in computational vision. J. Amer. Statist. Assoc., v. 82, p. 76-89, 1987.

[Masks(2005)] Masks, H. The largest site for halloween masks. 2005.

Disponível em http://www.halloween-mask.com/boingo \protect $\backslash \mathrm{T} 1 \backslash$ textunderscorelubati.htm

[Miller et al.(2003)] Miller, G. L.; Pav, S. E.; Walkington, N. J. When and why ruppert's algorithm works. In: Proceedings of the 12th International Meshing Roundtable, Sandia National Laboratory, 2003, p. 91-102.

Disponível em citeseer.ist.psu.edu/miller03when.html

[Minghim et al.(2002)] Minghim, R.; Nonato, L.; Batista, J.; Biscegli, C.; Franco, R.; Jorge, L. A. C. Three-dimensional visualization and volume determination from magnetic resonance imaging of mango diseases. Computers and Electronics in Agriculture, 2002.

[Nonato et al.(2005)] Nonato, L. G.; Cuadros-Vargas, A. J.; Minghim, R.; OliveirA, M. C. F. D. Beta-connection: Generating a family of models from planar cross sections. ACM Trans. Graph., v. 24, n. 4, p. 1239-1258, 2005.

[ParaView(2005)] PARAVIEw, K. Paraview: an open-source, multi-platform, extensible 
application designed for visualizing large data sets. 2005.

Disponível em http://www.paraview.org/

[Pav e Walkington(2004)] Pav, S.; Walkington, N. Robust three dimensional delaunay. In: Thirteenth International Meshing Roundtable, 2004.

Disponível em citeseer.ist.psu.edu/720156.html

[Pébay e Baker(2001)] PÉBAy, P. P.; BAKER, T. J. A comparison of triangle quality measures. Proceedings, 10th International Meshing Roundtable, Sandia National Laboratories, p. 327-340, 2001.

Disponível em http://www.imr.sandia.gov/papers/imr10/pebay01.ps.gz

[Pedrini(2001)] PEDrini, H. An improved refinement and decimation method for adaptive terrain surface approximation. In: Conference in Central Europe on Computer Graphics, Visualization and Computer Vision, 2001, p. 103-109.

[Pescatore et al.(2001)] Pescatore, J.; Bloch, I.; Baillet, S.; ET Al. Fem tetrahedral mesh of weed tissues from mri under geometrical and topological constraints for applications in eeg and meg. NEUROIMAGE, v. 13:(6), p. S218-S218 Part 2 Suppl., 2001.

[Planet(2006)] Planet, A. Animal planet. 2006.

Disponível em http://animal.discovery.com/

[Roettger(2006)] Roettger, S. The volume library - university of erlangen. 2006.

Disponível em http://www9.informatik.uni-erlangen.de/External/vollib/

[Roxborough e Nielson(2000)] Roxborough, T.; Nielson, G. M. Tetrahedron based, least squares, progressive volume models with application to freehand ultrasound data. In: VISUALIZATION '00: Proceedings of the 11th IEEE Visualization 2000 Conference (VIS 2000), Washington, DC, USA: IEEE Computer Society, 2000.

[Ruppert(1995)] RuppeRT, J. A delaunay refinement algorithm for quality 2-dimensional mesh generation. J. Algorithms, v. 18, n. 3, p. 548-585, 1995.

Disponível em citeseer.nj.nec.com/article/ruppert94delaunay.html

[Saitou e Nei(1987)] Saitou, N.; NeI, M. The neighbor-joining method: a new method for reconstructing phylogenetic trees. Mol Biol Evol, v. 4, n. 4, p. 406-425, 1987. 
[Schroeder et al.(1998)] Schroeder, W.; Martin, K.; Lorensen., W. The visualization toolkit: An object-oriented approach to 3d graphics. 2nd ed. Prentice-Hall, Englewood Cliffs, NJ, 1998.

Disponível em http://www.vtk.org/

[Shahidi et al.(1998)] Shahidi, R.; Wang, B.; M. Epitaux, E. A. Volumetric image guidance via a stereotactic endoscope. Lect. Notes Comput. Science, v. 1496, p. 241-252, 1998.

[Shewchuk(1999)] Shewchuk, J. Lecture notes on delaunay mesh generation. In: Acta Numerica, 1999.

[Shewchuk(2000a)] SHewchuk, J. Lecture notes on delaunay mesh generation. Relatório Técnico CA 94720, Department of Electrical Engineering and Computer Science - Berkeley, 2000a.

[Shewchuk(2002a)] SHEwCHuk, J. Delaunay refinement algorithms for triangular mesh generation. 2002a.

Disponível em citeseer.ist.psu.edu/shewchuk01delaunay.html

[Shewchuk(2004)] SHewCHuk, J. Triangle: A two-dimensional quality mesh generator and delaunay triangulator. 2004.

Disponível em http://www.cs.cmu.edu/〜 quake/triangle.html

[Shewchuk(2005)] SHEwCHuK, J. Theoretically guaranteed delaunay mesh generation-in practice (short course). In: Fourteenth International Meshing Roundtable, San Diego, California, USA, 2005.

[Shewchuk(1997)] Shewchuk, J. R. Delaunay refinement mesh generation. Tese de Doutoramento, School of Computer Science - Carnegie Mellon University, 1997.

[Shewchuk(1998)] Shewchuk, J. R. Tetrahedral mesh generation by delaunay refinement. In: Symposium on Computational Geometry, 1998, p. 86-95.

Disponível em citeseer.ist.psu.edu/shewchuk98tetrahedral.html

[Shewchuk(2000b)] Shewchuk, J. R. Mesh generation for domains with small angles. In: Symposium on Computational Geometry, 2000b, p. 1-10.

Disponível em citeseer.ist.psu.edu/269130.html 
[Shewchuk(2002b)] Shewchuk, J. R. What is a good linear element? - interpolation, conditioning, and quality measures. 2002b.

Disponível em citeseer.nj.nec.com/540424.html

[Sonka(1999)] SonkA, M. Image processing, analysis and machine vision. PWS Publishing, 1999.

[Stanford(2006)] STANFORD The stanford volume data archive - stanford computer graphics laboratory. 2006.

Disponível em http://www-graphics. stanford.edu/data/voldata/

[Stroustrup(2000)] Stroustrup, B. The $c++$ programming language. 3 ed. Addison Wesley Pub, 2000.

[Takahashi et al.(2004)] Takahashi, S.; Takeshima, Y.; Nielson, G.; Fujishiro, I. Topological volume skeletonization using adaptive tetrahedralization. In: Proceedings of Geometric Modeling and Processing, 2004, p. 227-236.

[Terzopoulos e Vasilescu(1992)] Terzopoulos, D.; Vasilescu, M. Sampling and reconstruction with adaptive meshes. In: IEEE Int. Conf. Comp. Vision, Pattern Recog., 1992, p. 829-831.

[Üngör(2004)] ÜNGÖR, A. Off-centers: A new type of steiner points for computing sizeoptimal quality-guaranteed delaunay triangulations. In: Proc. of the 6th Latin American Symposium on Theoretical Informatics (LATIN'04), Buenos Aires, Argentina, 2004, p. 152-161.

[UT(2006)] UT, T. U. o. T. Medical image databases on the internet. 2006.

Disponível em http://www.library.uthscsa.edu/

[Vieira et al.(2003)] Vieira, A. W.; Velho, L.; Lopes, H.; Tavares, G.; Lewiner, T. Fast stellar mesh simplification. In Proceedings of SIBGRAPI 2003 - XVI Brazilian Symposium on Computer Graphics and Image Processing. IEEE Press, 2003.

[Yan et al.(2005)] Yan, Z.; Kumar, S.; KuO, C. Mesh segmentation schemes for error resilient coding of 3-d graphic models. IEEE Trans. Cir. Sys. Video, v. 15, n. 1, p. 138144, 2005. 
[Yang et al.(2003)] YAng, Y.; Wernick, M.; Brankov, J. A fast approach for accurate content-adaptive mesh generation. IEEE Trans. on Image Processing, v. 12, n. 8, p. 866881, 2003.

[Zhang e Liu(2005)] Zhang, H.; LiU, R. Mesh segmentation via recursive and visually salient spectral cuts. In: Vision, Modeling, and Visualization, 2005, p. 429-436.

[Zhang et al.(2003)] Zhang, Y.; BAJAJ, C.; Sohn, B.-S. Adaptive and quality 3D meshing from imaging data. In: Proceedings of the eighth ACM symposium on Solid modeling and applications, 2003, p. 286-291.

[Zucker(2001)] ZuCKER, S. Relaxation labeling: 25 years and still iterating. In: DAVIS, L. S., ed. Foundations of Image Understanding, Kluwer Academic Publ, p. 289-322, 2001.

[Zuniga e Haralick(1987)] ZunigA, .; HarAlick, R. Integrated directional derivative gradient operators. v. SMC-17, p. 508-517, 1987. 